\title{
Fast VMZ: Code Enhancements for Video Mosaicing and Summarization
}

\author{
A Thesis presented to \\ the Faculty of the Graduate School \\ at the University of Missouri
}

In Partial Fulfillment

of the Requirements for the Degree

Master of Science

by

NAFIS AHMED

Dr. Kannappan Palaniappan, Thesis Supervisor

May 2021 
The undersigned, appointed by the Dean of the Graduate School, have examined the dissertation entitled:

Fast VMZ: Code Enhancements for Video Mosaicing and Summarization

presented by Nafis Ahmed, a candidate for the degree of Master of Science and hereby certify that, in their opinion, it is worthy of acceptance.

Dr. Kannappan Palaniappan

Dr. Filiz Bunyak

Dr. Abu Saleh Mohammad Mosa 


\section{Acknowledgements}

I would like to express my sincere gratitude and appreciation to my adviser Dr. Kannappan Palaniappan for his guidance and supervision. He has been supporting me throughout the whole journey of this research work with his patience and leadership. His continuous encouragement and inspiration has helped me to grow into a better researcher. I would like to extend my thanks to my committee member Dr. Filiz Bunyak and Dr. Abu Saleh Mohammad Mosa for making time out of their busy schedule and serving as my committee members. My sincere thank also goes to Rumana Aktar who has helped me with my research in every step of the way with her expert knowledge and constructive suggestions. Last but not least, my heartfelt gratitude goes to my wife who has been always very supportive about my research. I would also like to thank my father, mother and elder sisters who always inspired me to achieve my dreams. I am also indebted to my in-laws who have always loved and comforted me during this wonderful but laborious journey towards completing my degree. 


\section{Contents}

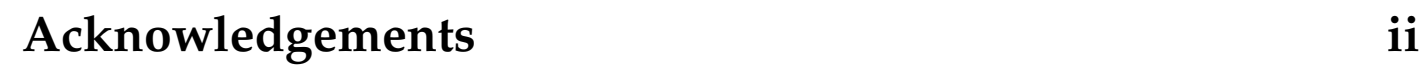

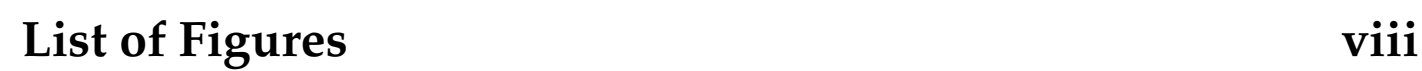

List of Tables $\quad$ xi

Abstract xii

\begin{tabular}{lll}
\hline Introduction & 1
\end{tabular}

1.1 Problem Statement . . . . . . . . . . . 1

1.2 Literature Review . . . . . . . . . . . . 2

1.3 Contributions ............... 5

1.4 Thesis Organization . . . . . . . . . . . . 6

2 Video Mosaicing and Summarization 8

$2.1 \quad$ Video Mosaicing and Summarization. . . . . . . 8

2.1 .1 Shot Detection . . . . . . . . . . . . . 9

2.1.2 Feature Detection and Matching . . . . . 9

2.1 .3 Homography Transformation . . . . . . . 10

2.1 .4 Warping and Blending . . . . . . . . . 11 
2.1.5 Implementation and Code Architecture of VMZ ................. 13

2.2 Performance Limitations of VMZ . . . . . . . . 16 2.2 .1 Code Profiling . . . . . . . . . . . 16

2.3 Modules selected for Speed-up . . . . . . . . . 20

3 Topics of Improvement for VMZ

3.1 Normalized Cross-Correlation . . . . . . . . . . . 24

3.2 Structure-Tensor . . . . . . . . . . . . . . . . . 25

3.3 Affine-Invariant SIFT . . . . . . . . . . . . . . 27

3.4 Blending . . . . . . . . . . . . . 30

$3.5 \quad$ Interfacing between MATLAB and $\mathrm{C}++\ldots . . . \quad 33$

4 Performance Enhancements and Speed-up of VMZ Mod-

ules $\quad 36$

4.1 Normalized Cross-Correlation . . . . . . . . . . . 36

4.1.1 CPU Based Multi-Threading . . . . . . . . 37

C++ Standard Threading Library . . . . . . 39

OpenMP ................ 42

4.1 .2 GPU Based Parallel Processing . . . . . . . 43

$4.1 .3 \quad$ MEX Interfacing $\ldots \ldots \ldots$. . . . . . . . 45

4.2 Structure-Tensor . . . . . . . . . . . . . . . . 47

4.2.1 Previous implementation details and timing 47

4.2 .2 GPU Based Parallel Processing . . . . . . . 48 
4.3 Affine-Invariant SIFT . . . . . . . . . . . . . . . . . 49

4.3.1 ASIFT - Redundant operations removed. . 52

$4.3 .2 \quad$ MEX Interfacing . . . . . . . . . . . . 55

$4.3 .3 \quad$ GPU Based Parallel Processing . . . . . . . 58

4.4 Proposed Blending Methods . . . . . . . . . . . . . 58

4.4.1 Blending Methods with Gradient condition 59

Center-Weighing Feather Blending . . . . . 60

Laplacian Pyramid Blending . . . . . . . . 60

4.4 .2 Illumination Matched Blending . . . . . . . 63

Proposed Illumination Difference Evalua-

tion Metric . . . . . . . . . . . 64

5 Experimental Result 66

5.1 Improved Normalized Cross-Correlation . . . . . 66

5.1 .1 Results of Improved NCC . . . . . . . . . 67

5.1 .2 Comparing the Output. . . . . . . . . 72

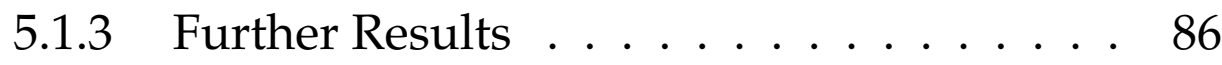

5.2 Improved Structure-Tensor Module . . . . . . . 88

$5.2 .1 \quad$ Dataset Used $\ldots \ldots \ldots$. . . . . . . . . . 88

5.2 .2 Hardware Used . . . . . . . . . . . . . 88

$5.2 .3 \quad$ Results for Improved ST . . . . . . . . . . . 88

5.3 Improved Affine-Invariant SIFT . . . . . . . . . . 93

$5.3 .1 \quad$ Dataset Used $\ldots . \ldots . \ldots 93$

5.3 .2 Hardware Used . . . . . . . . . . . . . . . 94 
5.3 .3 Results for Improved ASIFT . . . . . . . . . 94

$5.3 .4 \quad$ GPU Implementation Results . . . . . . . . 95

5.4 Proposed Illumination-Matched Blending . . . . . 99

5.4 .1 Dataset Used . . . . . . . . . . . . . . 999

5.4 .2 Results for Illumination-Matching . . . . . 99

5.4 .3 Evaluation . . . . . . . . . . . . 106

5.5 Overall Performance Improvement of VMZ . . . . 109

5.5 .1 Updated Code-Profiling . . . . . . . . . . 109

5.5.2 Updated Code Architecture for VMZ . . . 116

6 Additional Contributions and Future Directions 118

6.1 Additional Contribution . . . . . . . . . . . . . 118

6.1 .1 Meta-Mosaic Generation . . . . . . . . . . . 118

6.1.2 Sequential tiling and Multi-Threaded image reading for $\mathrm{VB} 3 \mathrm{D} \ldots \ldots 120$

6.1.3 Investigating PopSift and CudaSift for Benchmarking . . . . . . . . . . . 121

6.2 Future Directions . . . . . . . . . . . . . . 123

6.2 .1 Meta-Mosaic Generation . . . . . . . . . . . 123

6.2.2 ASIFT - GPU based implementation . . . . 124

6.2.3 Improved Structure Tensor - Integration with MATLAB $\ldots \ldots \ldots$. . . . . . . 125

6.2.4 Porting of VMZ MATLAB modules in C++ 125

6.2 .5 Camera Pose Estimation . . . . . . . . . . 126 
\begin{tabular}{ll}
\hline 6.2 .6 & Support for Deep and Engineered Features 127
\end{tabular}

\begin{tabular}{lll}
\hline 7 & Conclusions & 128
\end{tabular}

\begin{tabular}{ll}
\hline Bibliography & 131
\end{tabular} 


\section{List of Figures}

2.1 Different parts of the Mini-Mosaic Generation pipeline 12

2.2 Previous code architecture for VMZ . . . . . . . . 15

2.3 Code profiling for VMZ . . . . . . . . . . . . 18

$2.4 \quad$ Total time and percentage of time taken by each module in VMZ . . . . . . . . . . . . . . . . . . . . 19

2.5 Parallelization in CPU and GPU. . . . . . . . . . 23

3.1 Figure showing ASIFT tilt and rotation. . . . . . . 29

3.2 Replacement blending with Gradient-Response Ratio condition . . . . . . . . . . . 32

4.1 Multi-threaded row-interleaved assignment. . . . 41

4.2 Diagram showing IPOL-ASIFT pipeline with redundant calls . . . . . . . . . . . . . 51

4.3 Diagram showing ASIFT pipeline with no redundant calls . . . . . . . . . . . . . . . 54

4.4 Diagram showing ASIFT pipeline with separate feature-extraction and matching interface with no redundant calls . . . . . . . . . . . . 5 57 
4.5 Alpha Blending, Feather Blending and Laplacian Pyramid Blending . . . . . . . . . . . . . . . 62

5.1 Timing comparison between NCC methods . . . . 71

5.2 Histogram showing difference between NCC implementations . . . . . . . . . . . . . 74

5.3 NCC results showing correlation-matrix and output 78

5.4 NCC results showing correlation-matrix and output for randomly generated image . . . . . . . 80

5.5 NCC results showing correlation-matrix and output for randomly generated image with a flat patch 81

5.6 NCC speed-up for different workloads. . . . . . . 85

5.7 ST features comparison between MATLAB and GPU implementation . . . . . . . . . . . . . . . 90

5.8 ST features comparison between MATLAB and GPU implementation with top-150 selected features 92

5.9 Diagram describing the inefficiency of the ASIFT GPU pipeline . . . . . . . . . . . . . . . 9 98

5.10 Mosaics for Mesentery Sequence3 dataset . . . . . 101

5.11 Mosaics for Mesentery Sequence4 dataset . . . . . 103

5.12 Mosaics for Malaria dataset . . . . . . . . . . . 105

5.13 Improved Code Profiling after integrating improved modules . . . . . . . . . . . . . . . 110 
5.14 Improvement in Total Time required for VMZ modules . . . . . . . . . . . . . . . . 112

5.15 Speed-up for VMZ modules . . . . . . . . . . 113

5.16 Pyramid plot for Total-time per module before and after integrating improvements . . . . . . . . . . 114

5.17 Pyramid plot for Percentage of time per module before and after integrating improvements . . . . 115

5.18 Updated Code Architecture for VMZ pipeline . . 117

6.1 Block diagram for future GPU implementation of ASIFT . . . . . . . . . . . . . . 125 


\section{List of Tables}

5.1 Comparison of performance between Normalized Cross-Correlation implementations . . . . . . . . 70

5.2 NCC timing and speed-up for randomly gener-

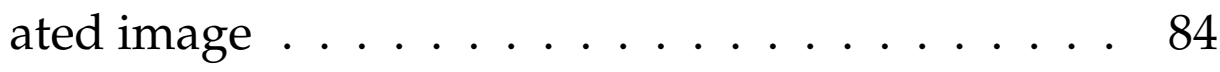

5.3 Time required and speed-up for Structure Tensor. 89

5.4 Time required and speed-up for ASIFT implementation over VMZ pipeline . . . . . . . . . . . 9 95

5.5 Time required and speed-up for ASIFT GPU . . . 96

5.6 Evaluation for mosaics created with different methods . . . . . . . . . . . . . . 107 


\section{Abstract}

Fast VMZ: Code Enhancements for Video Mosaicing and Summarization by Nafis Ahmed

Video Mosaicing and Summarization (VMZ) is a novel image processing pipeline that summarizes the content of a long sequence of geospatial or biomedical videos using a few coverage maps or mini mosaics. The existing VMZ algorithm uses Normalized Cross-Correlation (NCC), Structure Tensor (ST), AffineInvariant SIFT (ASIFT), Speeded up robust features for its feature matching and homography estimation pipeline, which are the most computationally expensive modules in the VMZ pipeline. Due to these long-running compute-intensive modules, the VMZ pipeline is not suitable for real-time mosaic formation in drones or UAVs. For instance, VMZ takes around 4 hours to generate mini-mosaics from an image sequence containing $9291 \mathrm{im}$ age frames. The blending algorithms used for mini-mosaic generation suffer from illumination variation due to the illumination difference in image frames. Such illumination inconsistency causes severe problems for biomedical scene understanding where curvilinear or tiny biological structures are present. VMZ pipeline is also dependent on 3rd party libraries not aligned with the flow of VMZ, which introduces redundant computation. One 
of the main reasons for the slow processing of the VMZ pipeline is not leveraging any parallel processing techniques and available graphics processing hardware. Therefore, the objective of this thesis is mainly three-fold: (i) speeding up the computeintensive and long-running modules in the VMZ pipeline, (ii) modifying the existing libraries and interfaces for better alignment with VMZ workflow, and (iii) resolving the illumination difference problem of the blending algorithms. Selected longrunning modules with the most impact on the overall run-time have been improved using CPU-based Multi-Threading, GPUbased Parallelization, and better integration with the existing VMZ pipeline. An illumination-matched blending algorithm has been proposed to improve the illumination problem. Besides, to evaluate the performance of different blending algorithms, a novel metric named Maximum Overall Illumination Difference (MOID) has been proposed. The improvement of VMZ modules has resulted in more than 100x speed-up in certain modules, with a $4.4 x$ speed-up for the total VMZ run-time. The novel illumination matched blending resulted in a better MOID value for image sequences not having illumination variance in a single frame. 


\section{Chapter 1}

\section{Introduction}

\subsection{Problem Statement}

Content summarization from video sequences is an exciting research domain of Computer Vision. The result of this pipeline is image mosaics, which can be a single mosaic or multiple mini mosaics from the given video sequence. The application of mosaicing includes a variety of fields like surveillance, medical imaging, monitoring of an urban area or agricultural field, and catastrophic events monitoring such as hurricanes, earthquakes, tsunamis, etc. [4]

Video Mosaicing and Summarization (VMZ) [2] pipeline can generate image mosaics from a given video sequence but takes a significant amount of time to create a single mosaic. The timeconsuming image processing modules of VMZ do not utilize any parallel processing, thus takes a long time for a single run. 
After the registration, the pipeline creates an image mosaic using replacement blending with an additional constraint to produce sharp image mosaics. Due to replacement blending, VMZ fails to address the illumination changes in different image frames. The existing evaluation metrics for mosaic uses only pixel-level correctness and structural similarity. None of these metrics considered the illumination changes in image frames.

Firstly, this thesis focuses on speeding up the compute-intensive modules of the VMZ pipeline that has the most impact on the overall run-time. Secondly, an illumination-matched blending algorithm has been proposed using the same gradient-based condition to produce sharp mosaics while addressing the illumination problem. Finally, a novel evaluation metric is proposed that can identify the illumination difference in a given mosaic.

\subsection{Literature Review}

There is a long history of image stitching techniques in the literature. A survey from Microsoft Research published in 2006 splits mosaicing into different types [17]. Video Mosaicing and Summarization, or VMZ, especially with Normalized Cross-Correlation (NCC), falls under the Feature-Based Registration techniques. Previous work on mini-mosaic generation considered reducing 
image drift [2, 3, 4] and also utilizes image geo-projection and stabilization.

Normalized Cross-Correlation (NCC) is a powerful feature matching tool. It has been used for quite some time, and so many methods have been proposed to speed it up for template matching. Lewis [14] proposed a method to speed it up using the pre-computed integral histogram, which many standard implementations take advantage of. Briechle and Hanebeck demonstrated NCC for template matching that achieves less computation by approximating the numerator [8]. In 2009, Yoo and Han introduced a significantly faster signal-processing-based method that takes advantage of logic operations to compute NCC without any multiplies [21]. This method is more sensitive to noise. However, Gangodkar et al., parallelized the Fast NCC (which is based on pre-computed sum-tables to mitigate the computational complexity of conventional NCC) on CUDA-based GPU[10]. NCC is parallelized across FPGA's by Wang and Wang, although the implementation is hardware-specific [20]. They showed the benefit of parallelizing the computation. In work on a tracker by Jakob Santner et al., they showed good results by also using the OpenCV NCC for the static part of their tracker, as it gives strong cues when the target reappears [16]. They did not analyze or parallelize the NCC. However, Arunagiri and Jaloma 
demonstrated that for stereo matching, NCC based cost function is the fastest and consumes less energy when implemented using integers rather than floating-point numbers on the GPU [6]. They did not try other methods of parallelization or focus specifically on NCC. Fouda and Ragab parallelized the NCC by using OpenMP [9] for shared-memory systems but did not explore the parallelization on GPUs. In this thesis, parallelization of Normalized Cross-Correlation for both CPU-based Multi-Threading and GPU-based parallel processing has been done [1]. The parallel NCC methods are also compared with its MATLAB counterpart function normxcorr2.

The four aspects of affine transformation, invariance to translation, rotation, and zoom, are dealt with by the SIFT [15] descriptor. The Affine-Invariant SIFT (ASIFT) descriptor [22] simulates a set of sample views of the initial images obtained by varying the two camera axis parameters. Thus ASIFT covers six aspects of the affine transformation. If the images in a given sequence are homogeneous, most of the feature descriptors except ASIFT fail to match good key points.

Mosaicing could be extremely challenging for biomedical video sequences having deformable structures. Deformable structures often cause motion blurring. Besides, large illumination changes affect the quality of blending which in turn hampers the mosaic. 
Significant research efforts are ongoing to encounter these challenges [5], to establish a robust registration algorithm. Featurebased registration was replaced in [5] with a Correlation-based registration method. Multiple blending techniques are compared in the paper [23] demonstrating that feather blending, Laplacian pyramid blending affects the illumination of the merged images better than the alpha blending, but this might cause blurring due to deformable structure for biomedical video sequences.

\subsection{Contributions}

The major contribution of this thesis include:

- A CPU-based Multi-Threaded algorithm for Normalized CrossCorrelation (NCC) capable of leveraging the computation power of multi-core CPUs. The improved module is more than 50x faster when integrated into the VMZ pipeline.

- An improved GPU-based implementation of Structure Tensor(ST), retaining the structure tensor response values to select the best feature points. This module is more than 100x faster for the same workload than the MATLAB tensor implementation currently being used in the VMZ pipeline.

- A modified the IPOL-ASIFT implementation for the VMZ pipeline to remove redundant feature detection operations. 
This results in a $4.4 \mathrm{x}$ speedup for the VMZ workflow of creating mini-mosaics. The dependency on file read/write has been removed by addressing the file input-output bottleneck for ASIFT.

- A new MEX interfacing developed to enable the VMZ MATLAB workflow to use the improved modules written in $\mathrm{C}++$.

- An Illumination-Matched blending algorithm for creating mosaics where different frames may have changes in illumination. A novel evaluation metric has also been proposed to determine the illumination difference in the generated mosaics.

- Integration of the improved Normalized Cross-Correlation and ASIFT module with the existing VMZ pipeline and have brought down the total run-time from 227 mins being taken previously to only 51.5 mins, achieving a speedup of $4.4 x$.

\subsection{Thesis Organization}

The chapters of this thesis is organized as follows:

Chapter 2 introduces the Video Mosaicing and Summarization workflow, and describes the task for each of the modules in VMZ. This chapter discusses the current code architecture of 
VMZ, as well as code profiling, finds the performance bottlenecks in the overall VMZ pipeline, and talks about methods that can be used for speeding up these compute intensive and long running modules.

Chapter 3 introduces the compute intensive modules that are targeted for speed-up as part of the work for this thesis. This chapter also introduces the blending algorithm currently being used in VMZ.

Chapter 4 discusses the methods used to achieve speed up for the targeted compute intensive modules. This chapter also describes the proposed illumination-matched blending method and the evaluation metric that is used to measure the illumination changes in a resulting mosaic.

Chapter 5 talks about the experimental result for the improved modules and analyzes over any unexpected outcome and differences between the improved modules and it's previous implementation.

Chapter 6 discusses additional contributions toward VMZ and other projects and proposes future extensions of the current work. Finally reasonable conclusions are drawn in Chapter 7. 


\section{Chapter 2}

\section{Video Mosaicing and}

\section{Summarization}

\subsection{Video Mosaicing and Summarization}

Image registration is one of the key problems in Computer Vision and a lot of research has been dedicated towards these from different domains. One of the basic approaches is to find the overlapping region between images with discernible key points using feature-based registration. The next step is to compute the Homography Matrix for applying the geometric transformations so that the images align in the same global coordinate. Generally the first image of the sequence is chosen as a reference for the global coordinate system. After computing the transformations, image frames are warped and blended together to create the resultant image mosaic with a holistic view of the region. 


\subsubsection{Shot Detection}

As the input video sequence can be very long running, it might be difficult to create a single image mosaic using the image frames from the input sequence. A difference of cumulative histograms is used to compute shot boundaries, that separates the whole input sequence into smaller segments, or shots. Once these shot boundaries are determined, the shots themselves can be identified automatically. A graph-cut algorithm is used to determine this shot boundaries in the VMZ pipeline.

\subsubsection{Feature Detection and Matching}

The first step in feature-based registration procedure is to find out distinctive feature points in images. Features can be corners, edges, contours, textures, intersection points, etc. If two images have some overlapping region, then there will be some common features, and these can be used to find the correspondence between images. Each of the well-known feature descriptors such as Harris corner, SIFT, PCA-SIFT, SURF, ASIFT, NCC, Structure Tensor, have their own strengths and weaknesses. For instance, Harris corner is an excellent corner detector but might not perform similarly for relatively homogeneous crop field data. SIFT shows promising results when comes to account for rotation, 
scaling and affine transformation while it is computationally expensive and poor at handling illumination change. On the contrary, SURF is usually faster with comparable performance to SIFT but can produce unsatisfactory results in the case of rotation and illumination change.

An upgraded version of SIFT and more robust descriptor is ASIFT which not only covers the four parameters from SIFT (scaling, orientation, illumination change, and affine distortion), but also use the latitude and longitude angles of the orientation of the camera axis, covering the six parameters of Affine Transformation. ASIFT introduced the notion of a transition tilt, which effectively measures the amount of distortion from one view to another using the camera axis parameters. If the images in a given sequence homogeneous then most of the feature descriptors except ASIFT fail to match good key-points. The main drawback of ASIFT is that it is few times computationally expensive than SIFT or SURF.

\subsubsection{Homography Transformation}

Once the image features are accurately detected and matched across frames, next step is to transform the sensed or warped image to overlay with the reference image. Early registration 
techniques evolved through translation, similarity, affine transformation to most general projective or Homography transformation.

\subsubsection{Warping and Blending}

Once the mapping function is generated, the final step is to transform the sensed or warped images. There are two ways to transform image pixels, forward and backward mapping. The first approach is forward transformation which directly maps image pixels using mapping functions, but creates holes in the output image. On the other hand, image pixels are redefined and interpolated using coordinates of reference system with inverse of mapping functions. A replacement blending method is used to overlay warped image on top of the reference image. This can be though of as a pixel replacement technique as each pixel in reference image is replaced by pixels the warped image. 

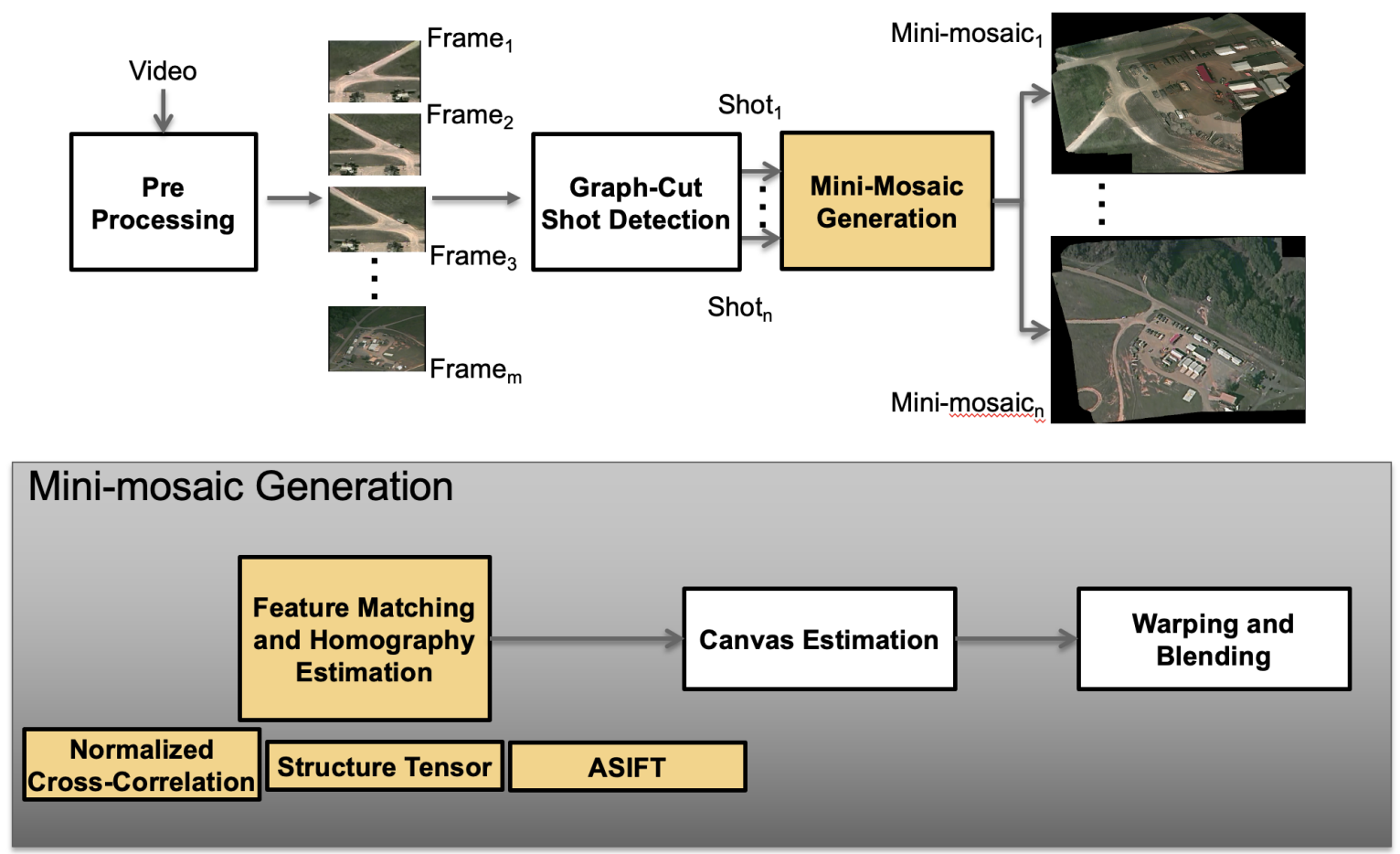

FIGURE 2.1: Figure showing the different parts of the pipeline for Mini-Mosaic Generation 


\subsubsection{Implementation and Code Architecture of VMZ}

Figure 2.2 shows the current code architecture of our VMZ pipeline.

Only the GraphCut operation in the pipeline that is being used for determining the shot boundary is implemented in $\mathrm{C}++$, and all the other modules are done in MATLAB. Though the IPOLASIFT code being used in implemented in $\mathrm{C}++$, the communication between the VMZ MATLAB pipeline and the ASIFT code is being done by reading from and writing to local files. This impacts the total run-time of the ASIFT operation being initiated from the VMZ pipeline significantly.

The VMZ pipeline starts with a parameterChecking function, that validates the provided parameter file, which includes the input video sequence, the output directory, and the runtime configurations and flags needed for initializing the different modules of VMZ. After the parameter file has been validated, the pipeline moves into computing the shotboundaries for the given input sequence. This is done using the GraphCut module already integrated with the VMZ.

After each of the shots are detected by identifying the shot boundaries, the VMZ pipeline starts its Feature Detection, Matching and Homography stage, which starts with the function MosaicInterface in the VMZ code base. This part mainly consists of four main functions, Current_to_Reference, 
Reference_to_Reference, Reference_to_Base, and Current_to_Base. The first two functions, Current_to_Reference, Reference_to_Reference, are where the main computational operations are done, including the feature detection and matching, and all four functions are used to estimate the planar homography for every frame with respect to the base frame, and using this homography estimation the mini-mosaics are created in the next stage of Warping and Blending, which starts with the function Warping ${ }_{B} l e n d i n g$ in the VMZ code architecture. 


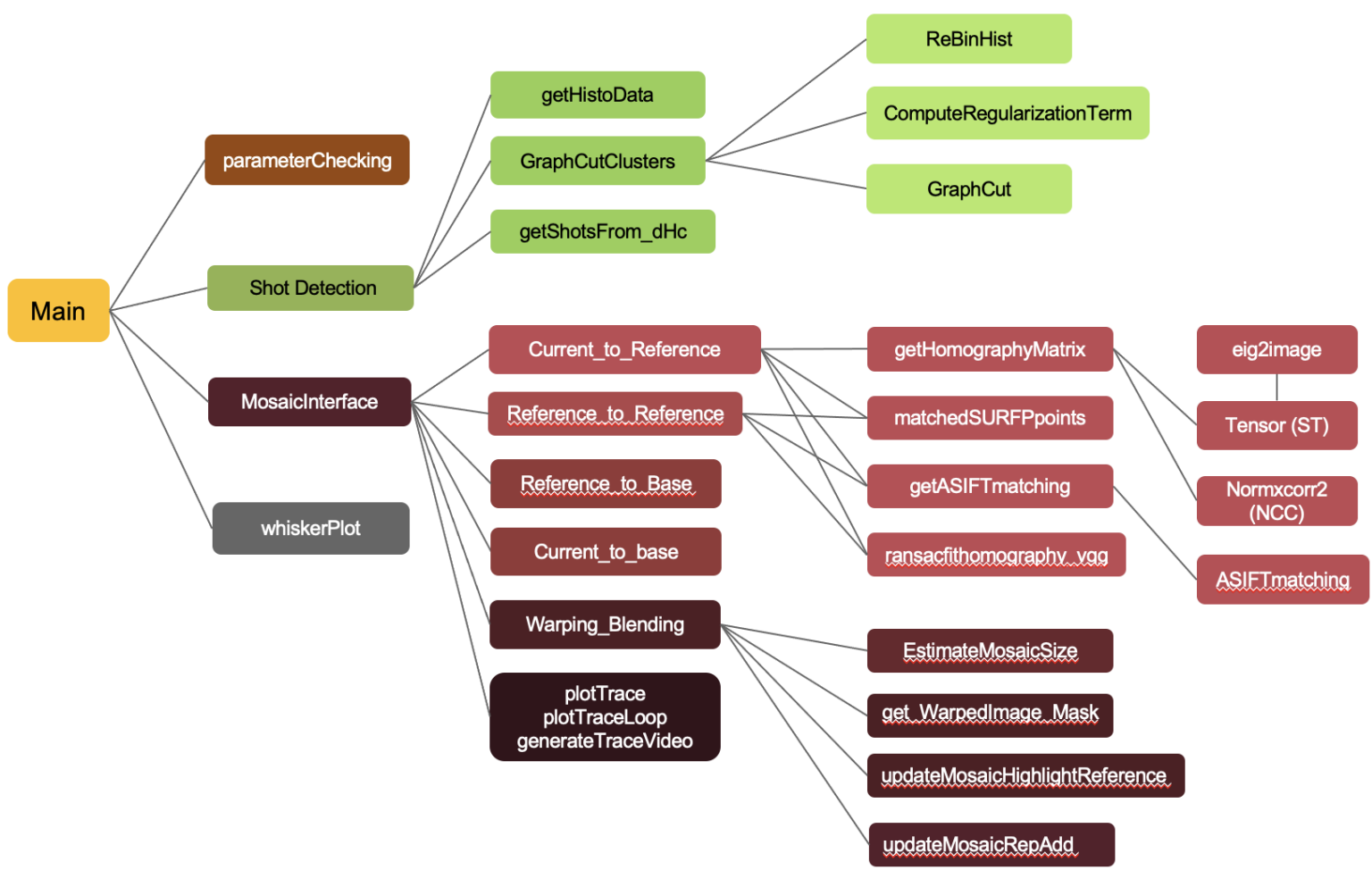

FIGURE 2.2: Figure showing the previous code architecture for VMZ, including all of the main MATLAB methods and operations in use. Courtesy: Internal methods are color coded by modules by Rumana Aktar 


\subsection{Performance Limitations of VMZ}

\subsubsection{Code Profiling}

We have timed the whole pipeline of $\mathrm{VMZ}$, starting from reading the images and ending at creating the image mosaics, to determine the most compute intensive module present in the VMZ, taking the most amount of time.

The whole pipeline has been divided into four operations for signifying a specific task in the pipeline, each containing multiple smaller operations themselves. They are:

1. Graph-Cut

2. Current-to-Reference Matching
a Structure-Tensor
b Normalized Cross-Correlation

3. Reference-to-Reference Matching a ASIFT

4. Warping and Blending

For the MATLAB implementation (version 1.28) of VMZ, creating the image mosaics from the dataset VIRAT tape2_8, which has 9291 image frames, took 5:46 hours or 335 minutes. Our code-profiling helped us in figuring out the individual tasks that 
takes the longest amount of time, so that we could work towards speeding up the slowest module first and having the most impact to the overall time for VMZ. By running the same workflow on the best available high performance cluster, containing Intel(R) Xeon(R) CPUE5-2680 v4 @ 2.40GHz server with 20 cores with Hyper-Threading, the mini-mosaics are generation took 3:36 hours or 227 minutes.

From the figure 2.3 we can see that the most compute intensive modules, thus taking the most amount of time, for the whole VMZ pipeline is the Normalized Cross-Correlation operation that is part of the Current-to-Reference block, that takes about $38.0 \%$ of the total runtime for VMZ, and the ASIFT feature extraction and matching operation, which is the part of the Reference-to-Reference block, taking about $49.7 \%$ of the total runtime for VMZ. And the third one, being the Structure-Tensor feature extraction operation of the Current-to-Reference block. This can be better visualized from the figure 2.4 below. 


\begin{tabular}{|c|c|c|}
\hline Total-Time & Pct & Operation \\
\hline \multicolumn{3}{|c|}{ 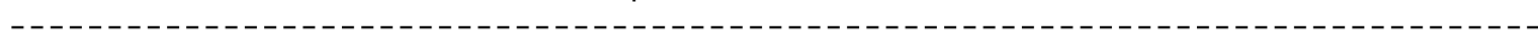 } \\
\hline 13579.50 & 100.00 & End-to-End $=9291 \mathrm{Fr}, 720 \times 480$ pixels \\
\hline 151.15 & 1.12 & GraphCut total time (reading $9291 \mathrm{fr}$ ) \\
\hline 6424.40 & 47.30 & Curr-Ref ST_NCC matching (reading 8184 fr) \\
\hline 5492.84 & 42.45 & Feature Extraction + Matching (Curr-Ref) \\
\hline 713.15 & 5.25 & Feature Extraction + Matching (ST) \\
\hline 4779.68 & 38.00 & Feature Matching (NCC in CPU) \\
\hline 297.87 & 1.20 & DLT + RANSAC (Curr-Ref) \\
\hline 6333.29 & 49.93 & Ref-Ref Xform by ASIFT (reading 251 REF $\mathrm{fr}$ ) \\
\hline 6246.81 & 49.63 & Feature Extraction + Matching (Ref-Ref) \\
\hline 81.71 & 0.30 & DLT + RANSAC (Ref-Ref) \\
\hline 669.77 & 4.93 & Warping+Blending (reading 8184 fr) \\
\hline
\end{tabular}

FIGURE 2.3: Code profiling for VMZ, showing the amount of time taken for each modules and the percentage of time being taken. The red boxes mark the two most compute intensive and time consuming modules for the VMZ pipeline 

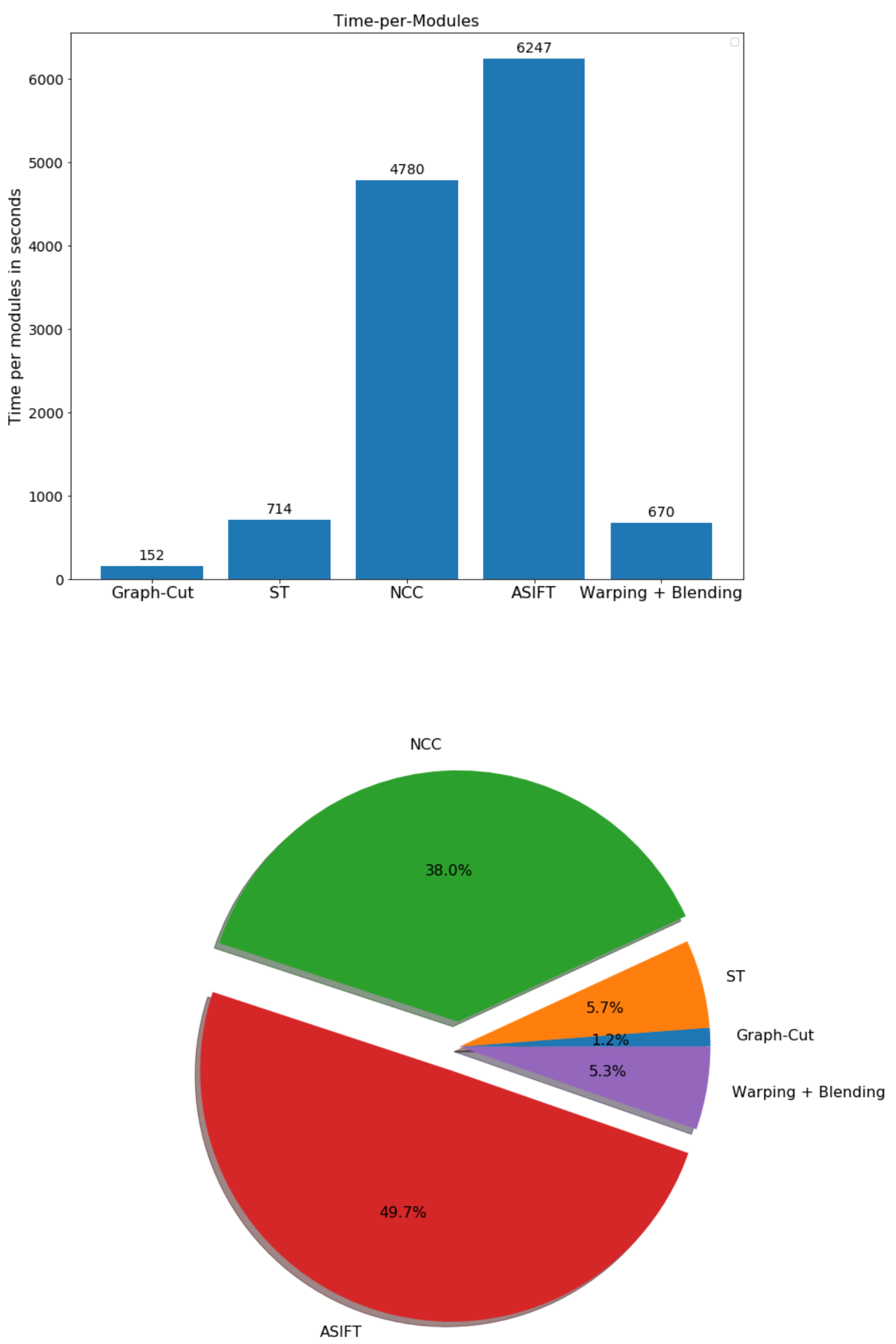

FIGURE 2.4: Figure showing total time and percentage of time taken by each module in VMZ. The bar plot above shows the time being taken per module in seconds, and the pie plot shows the percentage of time being taken by the modules. From these two plots, we can clearly identify the most time consuming and compute intensive modules in VMZ 


\subsection{Modules selected for Speed-up}

From the code-profiling of the MATLAB implementation for VMZ, it can be clearly seen that two of the most compute intensive operation is the NCC-Matching, taking $38.0 \%$ of the total required time, and the ASIFT operation, taking $49.7 \%$ of the total required time. Computing the Structure-Tensor is also one of operations that can be parallelized to gain speed-up. To reduce the overall required time to generate image mosaics using the VMZ pipeline, I focused on on the three most compute intensive modules, and by using parallelization techniques, gaining speed-up and reducing the overall required time for the whole pipeline. The selected modules are:

1. Normalized Cross-Correlation

2. ASIFT

3. Structure-Tensor

There are mainly two types of parallelization for gaining speedup,

1. CPU Based Multi-Threading

2. GPU Based Parallelization

- CPU Based Multi-Threading 
Modern operating systems can run multiple programs at the same time. Each program is known as a process that is being executed. The operating system knows many software tricks to make a process run along with others, as well as taking advantage from the underlying hardware. Running processes in an operating system is not the only way to perform several operations at the same time. Each process is able to run simultaneous sub-tasks within itself, called threads. It can be thought of a thread as a slice of the process itself. Every process triggers at least one thread on startup, which is called the main thread. Then, according to the program/programmer's needs, additional threads may be started or terminated. Multi-threading is about running multiple threads within a single process.

Each process has its own chunk of memory assigned by the operating system. By default, that memory cannot be shared with other processes and two or more processes have no way to share data, unless they perform advanced tricks - which is known as inter-process communication (IPC). Unlike processes, threads share the same chunk of memory assigned to their parent process by the operating system. Therefore, it is easier for two threads to talk to each other. On top of that, threads are usually lighter than a process, 
they take less resources and are faster to create, that's why they are also called lightweight processes.

Threads are a handy way to make your program perform multiple operations at the same time. Without threads you would have to write one program per task, run them as processes and synchronize them through the operating system. This would be more difficult, as using IPC is tricky, and slower, as processes are heavier than threads.

- GPU Based Parallelization

GPU follows a different parallel processing architecture than the CPU, which is known as Single-Instruction MultipleData or SIMD. This allows it to perform multiple calculations across streams of data simultaneously and can gain a significant boost in terms of speed-up over the CPU, where the data can be processed in parallel using the same instruction sets. 


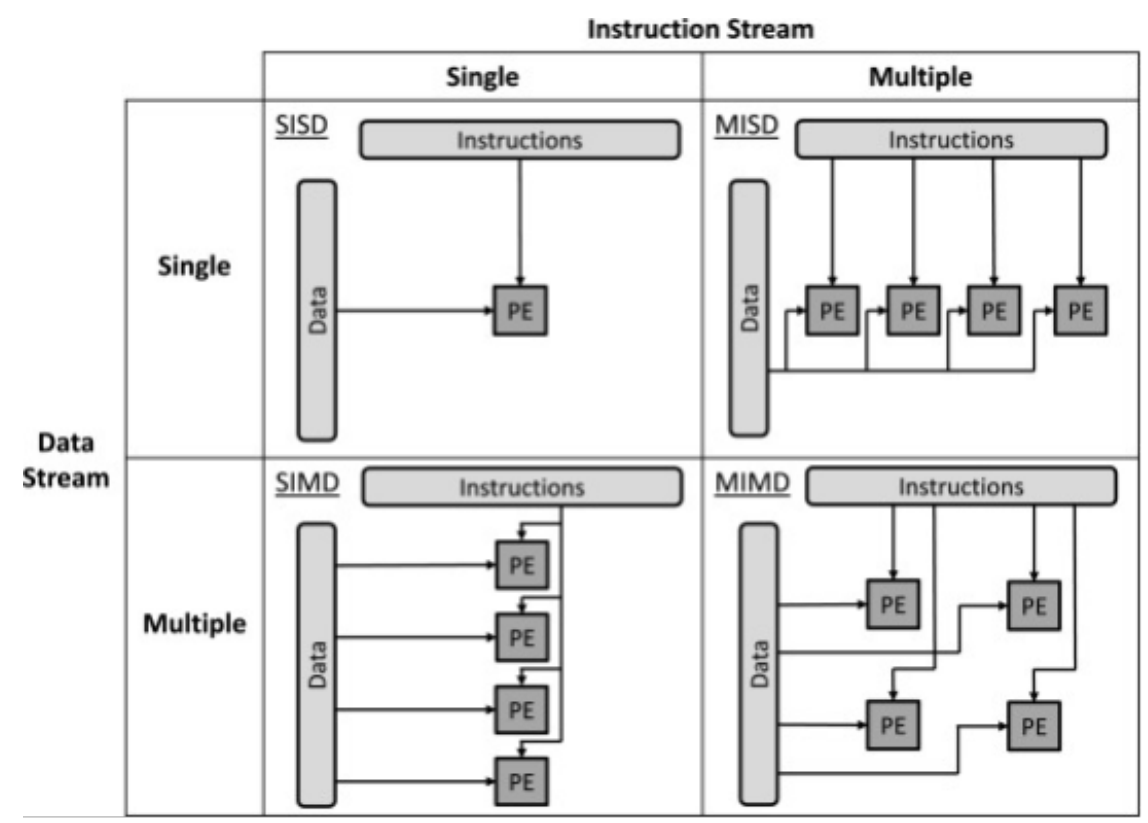

FIGURE 2.5: This figure shows how the parallelization methods differ between CPU multi-threading, which uses SISD processing, and GPU, which uses the SIMD processing. Some CPU support SSX, which gives it the option for performing SIMD operations, but the codebase might be bound to run in specific CPU architectures in that case 


\section{Chapter 3}

\section{Topics of Improvement for VMZ}

\subsection{Normalized Cross-Correlation}

Covariance provides a measure of the strength of the correlation between two sets of numbers or series. A serious setback of the covariance is its dependence on the amplitude of either of the numbers or the series that are compared. This dependency is eliminated if one uses the normalized form of the covariance, referred to as the normalized cross-correlation which is also known as the correlation coefficient.

Normalized Cross Correlation (NCC) is a popular measure of similarity between two images or image blocks. It is less sensitive to absolute intensity changes than other methods, but is quite expensive to compute [13].

The NCC between a template (or reference) image block $T(B, \mathbf{x}, t-$ $k$ ) from the frame at time $(t-k)$ and search (or current/source) image block $I(B, \mathbf{x}, t)$ from the frame at time $t$, is defined as 
$\gamma\left(B, \Delta \mathbf{x}_{B}, t\right)=\frac{\sum_{\mathbf{x} \in B}\left[T(B(\mathbf{x}), t-k)-\mu_{T}(B(\mathbf{x}), t-k)\right]\left[I\left(B\left(\mathbf{x}+\Delta \mathbf{x}_{B}\right), t\right)-\mu_{I}\left(B\left(\mathbf{x}+\Delta \mathbf{x}_{B}\right), t\right)\right]}{\sqrt{\sum_{\mathbf{x} \in B}\left[T(B(\mathbf{x}), t-k)-\mu_{T}(B(\mathbf{x}), t-k)\right]^{2} \sum_{\mathbf{x} \in B}\left[I\left(B\left(\mathbf{x}+\Delta \mathbf{x}_{B}\right), t\right)-\mu_{I}\left(B\left(\mathbf{x}+\Delta \mathbf{x}_{B}\right), t\right)\right]^{2}}}$

where $\mu_{B}(B, t-k)=\langle I(B, \mathbf{x}, t-k)\rangle$ and $\mu_{B}(B, t)=\langle I(B, \mathbf{x}+$ $\Delta, t)\rangle$ are the local intensity means in the target and template image regions, respectively [13].

\subsection{Structure-Tensor}

Structure tensors have been used in image processing to solve problems such as anisotropic filtering and motion detection. This method uses the gradient information of an image in order to determine the orientation information of the edges and corners. This method is represented by the gradient information. It also makes use of the difference between horizontal pixels (Ix) and vertical pixels (Iy) values, however, in a matrix form. The term "tensor" refers to a representation of an array of data.

The "rank" of a tensor denotes the number of indices needed to describe it. For example, a scalar value ' $\mathrm{X}^{\prime}$ is a single value, hence requires no indices. This means that a scalar is a tensor of rank zero. A vector, often represented as $\mathrm{Vi}=\mathrm{V} 1, \mathrm{~V} 2, \ldots, \mathrm{Vn}$ uses a single index $=\mathrm{i}$. This implies that a vector is a tensor of 
rank one. A two-dimensional matrix Mij is a tensor of rank two and so and so forth.

Image 2D Structure Tensor

$$
J_{2 D}(x, y)=\nabla I(\nabla I)^{T}=\left[\begin{array}{cc}
I_{x}^{2} & I_{x} I_{y} \\
I_{y} I_{x} & I_{y}^{2}
\end{array}\right]
$$

Image 2D Structure Tensor with Smoothing

$$
J_{2 D}(x, y)=G_{\sigma} * \nabla I(\nabla I)^{T}
$$

Eigen-decomposition is then applied to the structure tensor matrix ' $\mathrm{S}$ ' to form the eigenvalues and eigenvectors (L1, L2) and $(\mathrm{e} 1, \mathrm{e} 2)$ respectively. These new gradient features allow a more precise description of the local gradient characteristics.

Based on the two eigenvalues, local structures can be determined as one of three types:

- Constant areas

- Edges

- Corners

Although using gradient vectors in an image can determine the edge orientations too, there are some other advantages in using structure tensors compared to gradient vectors alone. First, 
the edges in an image may not be smooth and continuous, especially in down-sampled images. With the Gaussian filtering of the gradient vectors in a neighborhood, as seen in the definition of the structure tensor, one can acquire more robust and accurate estimation of edge orientations. Second, the structure tensor can classify local features into several distinctive types, which is nontrivial by using gradient vectors alone.

\subsection{Affine-Invariant SIFT}

Images obtained by cameras for object that has a smooth boundary from different positions goes through an apparent deformation. These deformations can be locally approximated by affine transforms of the image plane. This is one of the main reasons that the object recognition problem is often built on top of the computation of affine invariant features. The similarity invariance, which is an invariance to translation, rotation, and zoom, can be dealt with by the very popular SIFT method. Affine SIFT, also known as ASIFT, simulates a set of sample views of the initial images, obtainable by varying the two camera axis orientation parameters, also thought of as the tilt of the camera, which are not treated by the SIFT method. It then applies the SIFT method itself to all generated images from the various configurations. 
A fully affine invariant image matching algorithm needs to cover the 6 affine parameters. The SIFT method covers 4 parameters by normalizing rotations and translations and simulating all zooms out of the query and of the search images. As illustrated in Figure [figure - asift-configurations], ASIFT complements SIFT by simulating the two parameters that model the camera optical axis direction, both the original and simulated images are represented respectively by squares and parallelograms, and then applies the SIFT method to compare the simulated images, so that all the 6 parameters are covered. In other words, ASIFT simulates three parameters: the scale, the camera longitude angle and the latitude angle (which can also be thought of as the tilt) and normalizes the other three, which are translation and rotation. ASIFT can thus be mathematically shown to be fully affine invariant. 


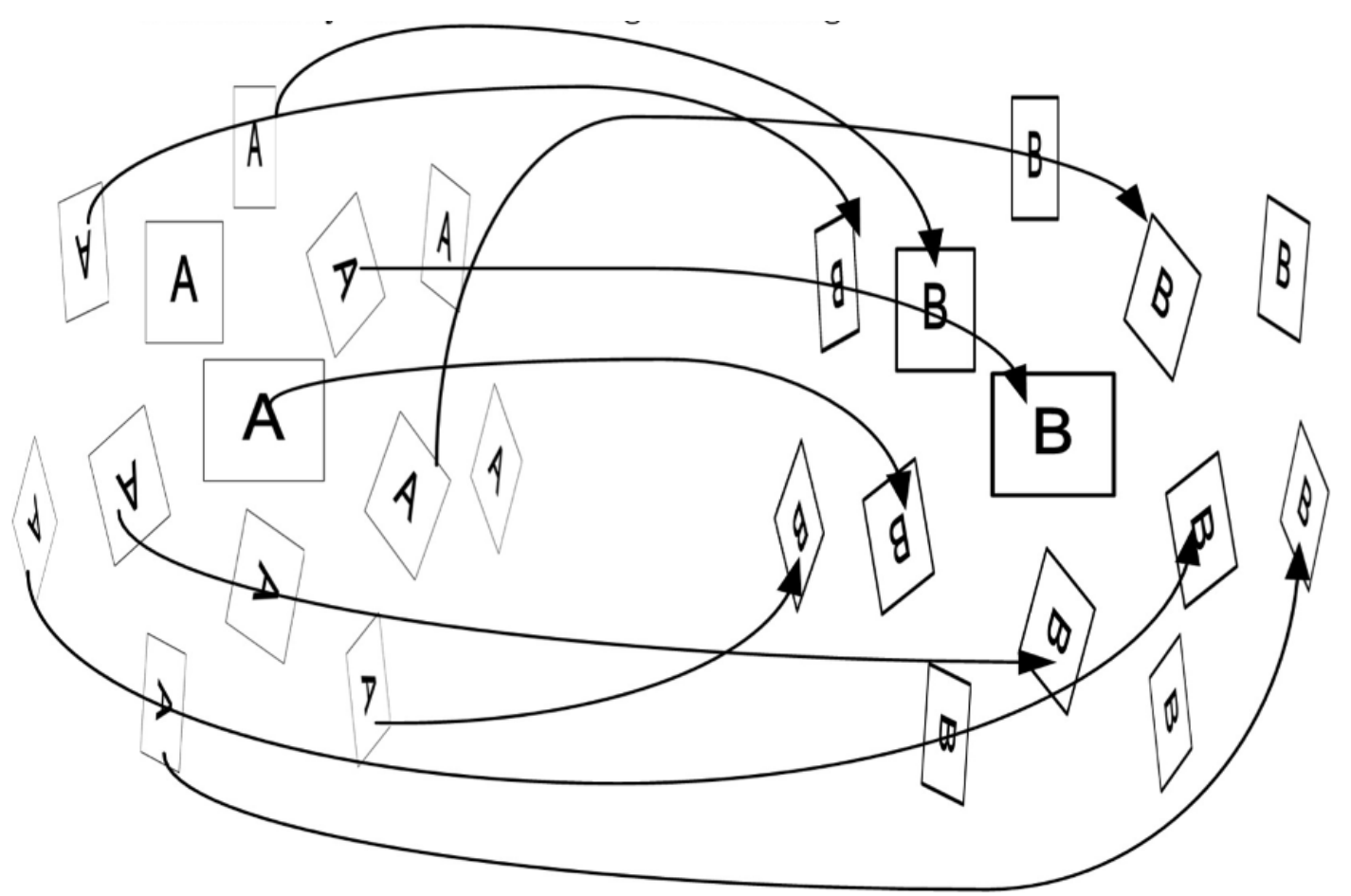

FIGURE 3.1: Figure showing the different tilt and rotation configurations for two images, from paper [22] 


\subsection{Blending}

After the registration step is complete for creating an image mosaic out of many image frames, different blending methods are applied on the overlapped regions on the images for generating the actual mosaic. The VMZ pipeline mainly uses replacement blending, that is a variant of a very well known algorithm named Alpha Blending. Alpha Blending uses a predetermined value, $\alpha$, that is multiplied with the overlapped pixels of the new image frames and the opposite $(1-\alpha)$ values is multiplied to the overlapped pixels of the mosaic.

To ensure a sharp mosaic when blending images to form the mosaic, VMZ uses a gradient-based blending algorithm. A Gradient Response Ratio (GRR) is computed, which is the ratio of gradient or edge information between the current image frame, and the previous frame. If this ratio has a value greater than 1 , the new image frame has sharper structures than the previous image, and thus the overlapped part of the mosaic is replaced by the new frame.

Otherwise only the new part that wasn't part of the mosaic is added from the new frame, and the overlapped part remains as it was. But as this technique uses the replacement blending, it has trouble blending in the mosaic when illumination change 
occurs, and the resultant mosaic shows a clear separation between lighter and darker images from the sequence. 

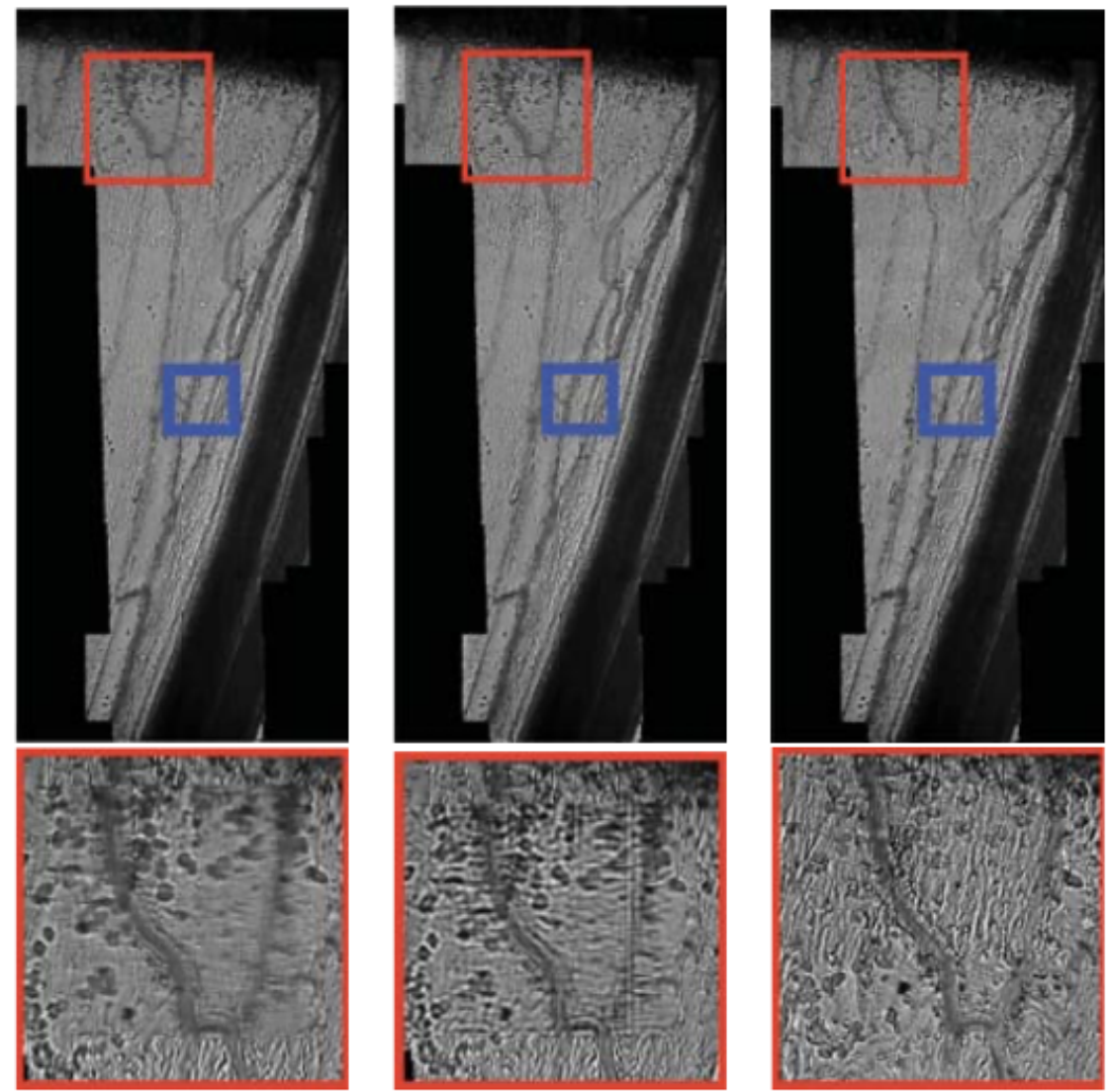

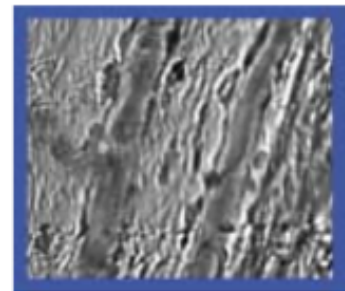

(a) Averaging

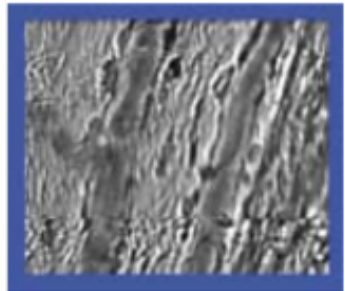

(b) $\mathrm{ADB}$

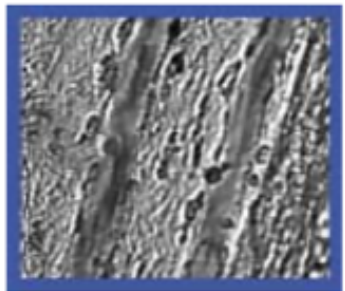

(c) Gradient

FIGURE 3.2: Figure showing Replacement blending with Gradient-Response Ratio condition compared with Alpha blending (averaging) and ADB, from paper [5] 


\subsection{Interfacing between MATLAB and C++}

Without using an interfacing between MATLAB and C++ modules, we would need to fallback to using file read/write to communicate between the different modules and the disk read/write speed will become the bottleneck. Interfacing between MAT$\mathrm{LAB}$ and $\mathrm{C}++$ plays a crucial role in speeding up individual modules of the VMZ MATLAB pipeline. As using $\mathrm{C}++$ also gives access to leverage the full potential of the multi-core CPU using multi-threading.

MEX Interfacing is used to create MATLAB functions that provide the efficiency of $\mathrm{C}++$ programs and it can access external functions and libraries from MATLAB. MEX interface integrates seamlessly into MATLAB, getting inputs from and returning results to MATLAB, and supports MATLAB data types and copy-on-write semantics. Using that it's possible to write thread-safe multi-threaded MEX functions, and all of the $\mathrm{C}++$ functions executes out-of-process.

There are three main parts in creating a MEX system:

1. C++ function

2. MATLAB source file

3. MEX Gateway function 
The $\mathrm{C}++$ function is where the $\mathrm{C}++$ module for the target work should be, and serves as the beginning of the $\mathrm{C}++$ module for the MEX system. The MATLAB source file is the exit point, from which point MATLAB starts the call into the MEX system. The MEX Gateway function serves as the middleware between the MATLAB and the $\mathrm{C}++$ codes.

MEX Gateway function acts as the entry point into the $\mathrm{C}++$ function, and it has a very specific method signature that needs to be populated in order to use the actual $\mathrm{C}++$ module from MATLAB. The method signature is, 'void mexFunction(int nlhs, mxArray *plhs[], int nrhs, const mxArray *prhs[])', where the nrhs and nlhs parameter denotes number of the input and the output parameters for the function, and prhs and plhs denotes the input parameter being passed from MATLAB and output parameter to be returned to MATLAB. This function can be completed by following four key steps:

- Reading Input Data: Reading the input data from the prhs parameter is the initial step of the MEX function. Using this the $\mathrm{C}++$ function can retrieve all of the input data passed from the MATLAB endpoint.

- Prepare Output Data: Memory needs to be allocated for the plhs parameter, where the output data will be stored within 
the gateway function. This data will automatically be returned to the MATLAB function once the function ends it's computation.

- Perform Computation: This is the step where the C++ module does all of its computation, here it can leverage the multithreading support or call any other $\mathrm{C}++$ library functions.

- Populate Output Data: After getting the result from the previous step, the final step is to populate the plhs data that is already allocated. One thing to note is that the gateway function doesn't need to return the output data explicitly out of the function, the MEX interfacing automatically retrieves the populated data inside the plhs variable, and sends it back to the MATLAB function once the gateway function completes its execution. 


\section{Chapter 4}

\section{Performance Enhancements and Speed-up of VMZ Modules}

\subsection{Normalized Cross-Correlation}

As seen from the profiling discussed in the previous sections, Normalized Cross-Correlation is the operation that uses more than half $(38.0 \%)$ the time of the total time required to run the VMZ pipeline. Reducing the runtime for this module will have the most impact on the total runtime, which is why this was the first module we looked at for gaining significant speedup. We have looked into both CPU based multi-threading and GPU based parallel processing (leveraging the SIMD architecture) as methods that are able to reduce the required time for the NCC module. 


\subsubsection{CPU Based Multi-Threading}

There are a few good libraries upon which a multi-threaded CPU implementation can be built, like Boost, OpenMP, Pthreads, etc. We have selected two widely used library and implemented Multi-Threading using both of them. They are:

- C++ Standard Threads (std::threads)

\section{- OpenMP}

One of them are standard $\mathrm{C}++$ threads, which was built on top of Pthreads, and introduced to the standard C++ library with the release of $\mathrm{C}++11$. The other multi-threaded implementation has been done with OpenMP, which is widely used library when it comes to multi-processing in $\mathrm{C}++$ and Fortran.

For both of the implementation, we have created a function which takes a reference patch of an image, and a search window patch, or two images, the first one is the reference image and the second one is the current image which will be compared with the reference block, coupled with the reference block coordinates and the search window coordinates, and outputs the coordinate for the best match using the normalized cross correlation and it's normalized cross correlation score.

For calculating NCC with the multi-threaded CPU implementation, we have used the matchTemplate [18] function from the 
well known and widely used OpenCV library, and we have used OpenCV v3.4.8 for our implementation. The matchTemplate function call has most widely known optimization, i.e., Integral Image usage, already implemented inside it, and has various methods for calculating NCC, which can be selected while calling the function. For our purpose, we have used the method CV_TM_CCOEFF_NORMED [18] for calculating NCC with the matchTemplate function. The matchTemplate function takes two images, the first one is the Input (i.e., Current) image, the second image is the Reference (i.e., Template) image, and the method, which will be used to calculate the NCC matrix. The output of this function is a correlation matrix, from which we can determine the best matched block from the scores from each cell of correlation matrix. If $I$ is the source image, $T$ is the template image, and $R$ is the correlation matrix, then the equation for NCC with the method CV_TM_CCOEFF_NORMED [18] becomes: 


$$
R(x, y)=\frac{\sum_{x^{\prime}, y^{\prime}}\left(T^{\prime}\left(x^{\prime}, y^{\prime}\right) \cdot I^{\prime}\left(x+x^{\prime}, y+y^{\prime}\right)\right)}{\sqrt{\sum_{x^{\prime}, y^{\prime}} T^{\prime}\left(x^{\prime}, y^{\prime}\right)^{2} \cdot \sum_{x^{\prime}, y^{\prime}} I^{\prime}\left(x+x^{\prime}, y+y^{\prime}\right)^{2}}}
$$

where,

$$
\begin{aligned}
T^{\prime}\left(x^{\prime}, y^{\prime}\right) & =T\left(x^{\prime}, y^{\prime}\right)-\frac{1}{(w \cdot h)} \sum_{x^{\prime \prime}, y^{\prime \prime}} T\left(x^{\prime \prime}, y^{\prime \prime}\right) \\
I^{\prime}\left(x+x^{\prime}, y+y^{\prime}\right) & =I\left(x+x^{\prime}, y+y^{\prime}\right)-\frac{1}{(w \cdot h)} \sum_{x^{\prime \prime}, y^{\prime \prime}} I\left(x+x^{\prime \prime}, y+y^{\prime \prime}\right)
\end{aligned}
$$

The details regarding how the multi-threading is done with the two CPU based threading libraries are described below:

\section{C++ Standard Threading Library}

The implementation with standard $\mathrm{C}++$ threads uses a row-interleaved technique for work distribution between the threads. The total threads can be of any number, but it results in the best performance when it is equal to the number of CPU threads. The rowinterleaved technique can be descried as follows. If the total number of threads are $\mathrm{T}$, and there are a total of $\mathrm{N}$ blocks, then the first block is assigned to the first thread, the second block is assigned to the second threads, and likewise the first $\mathrm{T}$ blocks are assigned to each of the T threads to perform the NCC calculation, then the $T+1$ block is again assigned to the first thread, 
and assignment goes on like this, with the final block of $\mathrm{N}$ is assigned to the thread $(N \bmod T)+1$. 

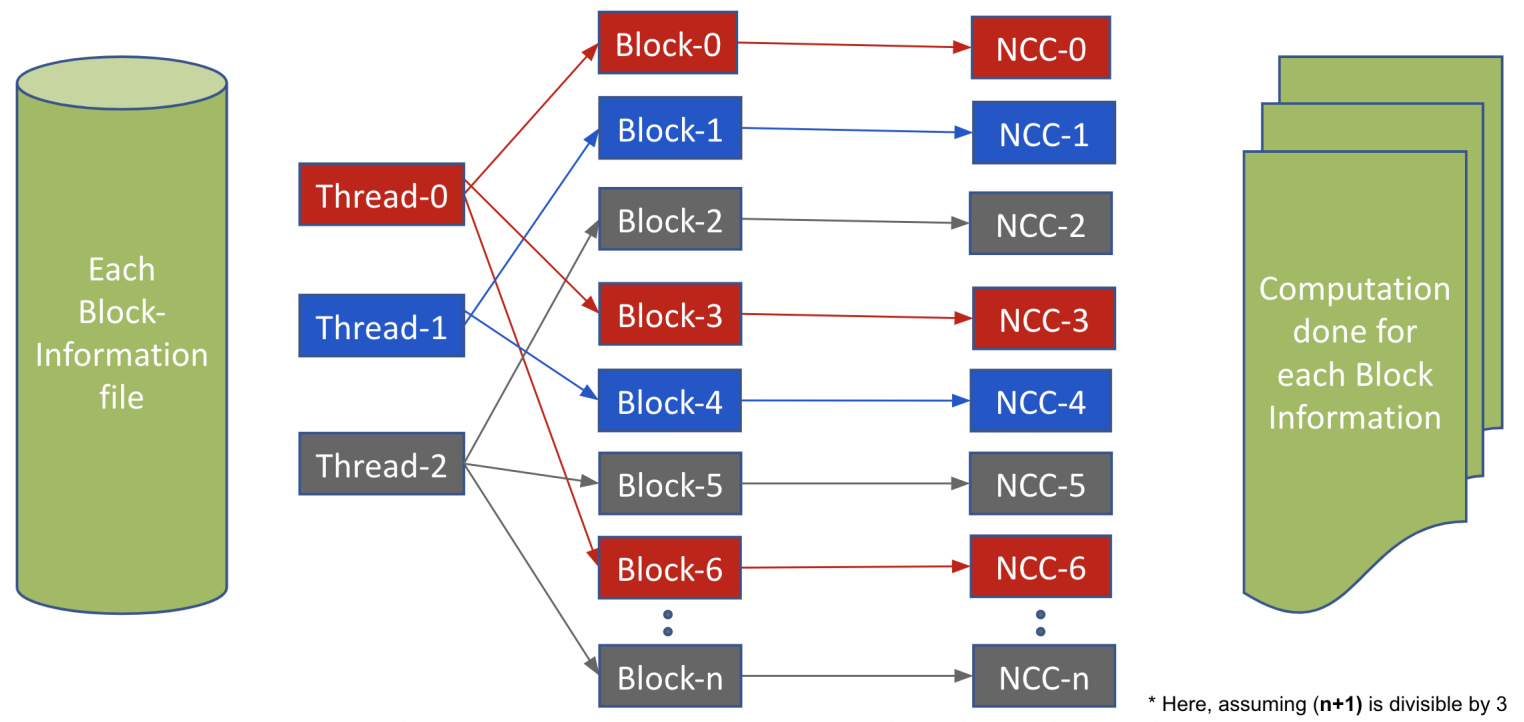

FIGURE 4.1: This shows the row-interleaved assignment technique. Each block's NCC calculation is done by a single predetermined thread, so no redundant calculation is done. If there are a total $t$ number of threads, each having an id, tid, ranging from 0 to $(t-1)$, then each thread is responsible for calculating the blocks, tid, $(t i d+t)$,

$\left(\right.$ tid $\left.+2^{*} t\right)$, and so on. 


\section{OpenMP}

OpenMP is a shared memory multi-processing programming interface, which uses a set of compiler directives, routines and environment variables to influence the runtime behavior of a program. For the implementation using OpenMP, we have used the same function to calculate the coordinate with the best NCC score, and initiated it's call from a standard for-loop which has been preceded by the OpenMP 'for' directive. We have used the specification 'schedule(static, 1) nowait' for this particular directive, so that it's task assignment to threads is similar to the implementation with the $\mathrm{C}++$ standard library. Here, the 'static' keyword implies that each of the threads will be statically assigned to a core. Also, a chunk size of 1 is used, so that each block of NCC calculation is considered as a single chunk of work. The 'nowait' keyword is used to speedup of calculation, as we don't need the threads to synchronize after each iteration.

For both of the implementation, the algorithm 1, remains the same, as only the multi-threading part is different for the two implementation with the two multi-threading libraries. 


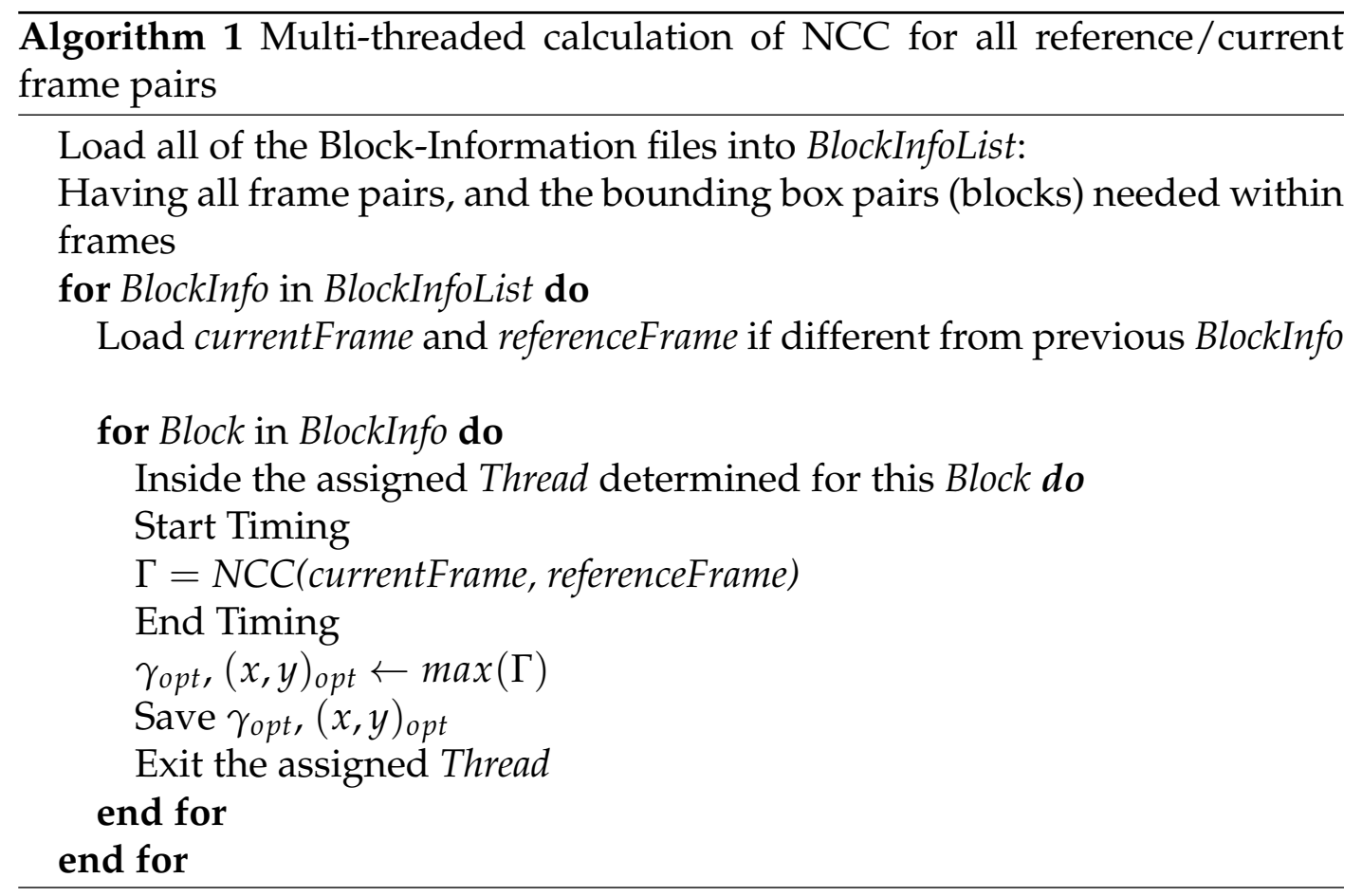

\subsubsection{GPU Based Parallel Processing}

For the GPU implementation, we use Nvidia Performance Primitives (NPP), a CUDA API that allows for easy CUDA programming. The various APIs handle tasks on the GPU to accelerate performance. NPP uses managed memory to make it easier for the programmer to transfer data to and from the GPU and use their APIs on the GPU. Our implementation must load the relevant images onto the GPU's memory.

To test the GPU as a comparison to the multi-threaded OpenCV versions, we use the NPP API'nppiCrossCorrValid_NormLevel_32f_C1R'. This returns the correlation matrix, from which we can compute the max. This is still on the GPU, so we use 'nppiMax_32f_C1R'. Both of these functions require use of buffers which use GPU 
memory, so there is some slight memory overhead for allocating the right amount of space for the correlation matrix and the necessary buffers.

$\overline{\text { Algorithm } 2 \text { GPU Calculation of NCC Coefficients for all reference/current }}$ frame pairs

Load all csv files into BlockInfoList: Know all frame pairs, and the bounding box pairs (blocks) needed within frames.

for BlockInfo in BlockInfoList do

Load referenceFrame if different from previous BlockInfo referenceFrame

Load currentFrame if different from previous BlockInfo currentFrame

Allocate all necessary space on the GPU, including with $\gamma_{o p t}$ with size (currentFrame.width - referenceFrame. width +1 , currentFrame.height - referenceFrame.height +1 )

Start Timing

$\Gamma=N C C$ (currentFrame, referenceFrame)

End Timing

$\gamma_{o p t}=\max (\Gamma)$

Save $\gamma_{\text {opt }}$ and $\Gamma$ to compare against current CPU results.

Free GPU resources, unless they are the right size to be reused in the next calculation.

end for

Algorithm 2 shows our pseudocode for the GPU implementation. Importantly, our timing throughout this paper has been focused on the actual NCC calculation. There may be some overhead associated with GPU implementation and integration (mainly) due to data staging in/out, but we wanted to analyze only the timing incurred by computing the NCC's for now.

We also tested OpenCV's GPU support for the matchTemplate function. This was done on a GeForce GTX 1080 with OpenCV 3.4 compiled with CUDA 10.1 support. 


\subsubsection{MEX Interfacing}

Previously, for computing the Normalized Cross-Correlation with a given template image and a source image, the built-in MATLAB function normxcorr 2 was being used, which gave the correlation matrix as an output. And from this correlation matrix, VMZ had a workflow to select the highest peak that denotes the best matched point of the template image within the source image. We have implemented our own multi-threaded module for computing this Normalized Cross-Correlation workflow, which has been discussed above. But the problem we face now is to call this $\mathrm{C}++$ multi-threaded method from our existing MATLAB pipeline. If calling the method directly using some kind of interfacing or inter process communication is not used then we will have to use file input/output as the communication method. Any speed-up of the NCC module will be bottle-necked by the read-write speed of the hard drive, and this will negatively affect the time required for any operations being done to compute the Correlation Matrix using NCC or to find the best matching point in the source image.

For overcoming this read-write bottleneck, I have leveraging the MEX file-function available for binding $\mathrm{C}++$ libraries 
with MATLAB through a custom written interface. For the Normalized Cross-Correlation module, I have created a MEX function with two main parameters which are the two images, a template image with a block, where the block specifies the actual template we will be searching for, and a source image paired with a search - window, which specifies the location within the source image in which we will be searching for the specified template block.

The MEX interfacing implemented for binding the C++ MultiThreaded NCC module with the VMZ MATLAB has one method, which as described above takes in two images, each paired with a block information list, where each element of the list denotes a template and a search - window. The method also takes in

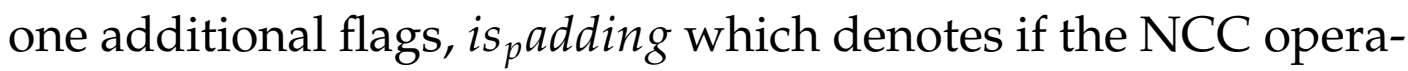
tion will be compute the correlation matrix with a zero padding around the edge, two threshold values, ncc $_{s}$ core $_{t}$ hreshold, which removed any matched block that has a score below the given threshold, and rangePct, which applies a suppression of a maximal point if any of the neighboring point's peak value comes within the provided range. For the last parameter, the method

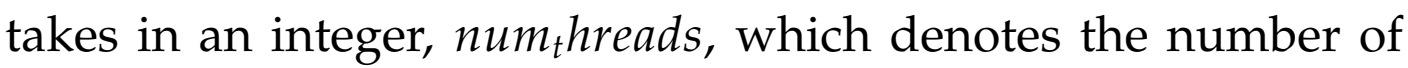
threads that is to be spawned for computing the NCC operations. 


\subsection{Structure-Tensor}

As seen from the profiling discussed in section 3, Structure Tensor operation uses around $5.2 \%$ the time of the total time required to run the VMZ pipeline. As described in the introduction section for Structure Tensor, this type of operation can leverage the power of SIMD processing (Single Instruction - Multiple Data) using GPU based parallel processing, and significant performance gain can be expected when starting from a single threaded implementation of such operation. Which is why we have looked into GPU based parallel processing (leveraging the SIMD architecture) as a possible method that should be able to reduce the required time for the ST module, and thus reducing the overall runtime of our VMZ pipeline.

\subsubsection{Previous implementation details and timing}

The structure tensor method previously used for our VMZ pipeline is based on MATLAB's built-in operations like "conv2" and "fspecial", which are fairly compute intensive processes themselves.

For computing the structure tensor features for a single image the MATLAB workflow took 7.35 milliseconds. This timing was averaged from a test where the same image was processed through the structure tensor pipeline 1000 times. 


\subsubsection{GPU Based Parallel Processing}

For the GPU implementation, we use Nvidia Performance Primitives (NPP), a CUDA API that allows for easy CUDA programming. The various APIs handle tasks on the GPU to accelerate performance. NPP uses managed memory to make it easier for the programmer to transfer data to and from the GPU and use their APIs on the GPU. Our implementation must load the relevant images onto the GPU's memory.

For computing the Structure-Tensor features, first we initialize our structure tensor class with five scale values, which is also known as the sigma value, of $3,5,7,9$, and 11 . For each such sigma value, we use the corresponding gaussian kernel and compute the Ix and Iy values for every pixel of our image. These two operations are computed using the correct filter and leveraging the CudaHelper methods "applyFilter_Row_32f" and "applyFilter_Column_32f".

After computing the Ix and Iy values in the first step, our next kernel computes the response value from the structure tensor matrix for every pixel of the image. This operation is followed by only keeping the maximal response value in a fixed $3 \times 3$ neighborhood.

To determine the top $K$ number of features from the feature point list, we need to retain this response value that is being 


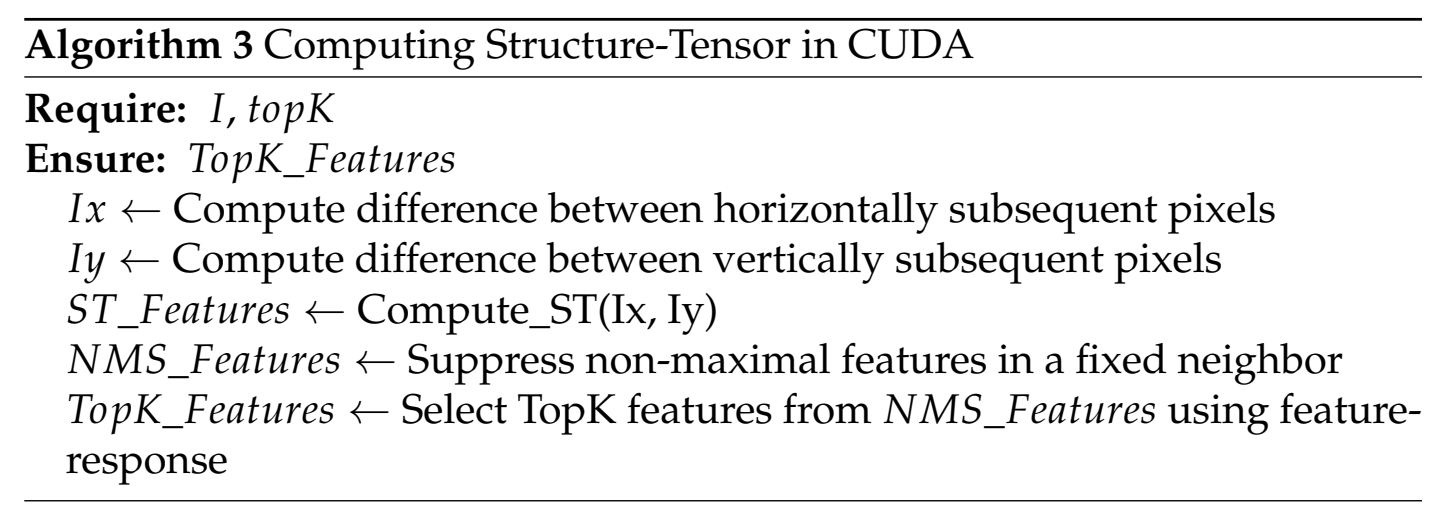

computed. Previously, only the scale value (sigma value) was being stored as part of the internal data structure, which is why no selection based on the strongest feature points was possible. For the improved implementation, this response value is made to be a part of the internal data structure being kept for every structure tensor feature point, and after extracting the feature points, a sorting algorithm is used to store the feature points based on the response value in a descending order. From that sorted list the top $K$ feature points are then returned as the result of the detect operation, where the $K$ value is taken from the caller of this method as a method-parameter.

\subsection{Affine-Invariant SIFT}

As seen from the profiling discussed in Chapter 2, Affine-Invariant SIFT, or ASIFT operation uses $49.7 \%$, or almost half of the time of the total time required to run the VMZ pipeline. This is the most compute intensive process being computed in the VMZ 
pipeline, and any speed-up for this particular module will impact the overall time required for VMZ pipeline significantly.

The ASIFT method currently used in the VMZ pipeline is the implementation from the IPOL-ASIFT [22] paper. We have looked at how this implementation is being interfaced with the VMZ pipeline, and removed redundant operations that was present in the pipeline due to having only a single method for performing both key-point detection and matching operations, which causes redundant computation for the feature-extraction process for an image, when the same image is encountered multiple times. 


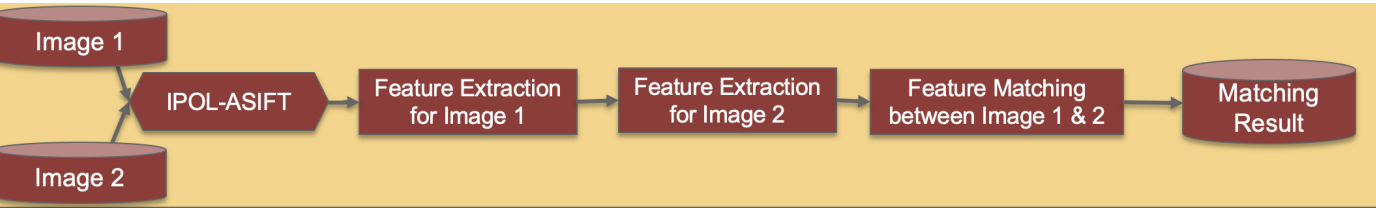

\section{IPOL-ASIFT Redundant Calls}

ASIFT("imageDir/img1.png", "imageDir/img2.png", "matching12.txt", ...) match12 = fileread ("matching12.txt"):

ASIFT("imageDir/img2.png", "imageDir/img3.png", "matching23.txt", ...);

match23 = fileread("matching23.txt");

ASIFT("imageDir/img3.png", "imageDir/img4.png", "matching34.txt", ...)

match34 = fileread ("matching34.txt");

FIGURE 4.2: Block diagram showing the original IPOL-ASIFT pipeline for feature extraction and matching, the function calls shown below underlines the images in red, which has redundant feature extraction computation 
We have also looked into interfacing this C++ ASIFT implementation with our MATLAB pipeline, leveraging the MEX interfacing available for binding $\mathrm{C}++$ libraries as MATLAB functions.

The IPOL-ASIFT implementation uses the OpenMP parallel processing library for leveraging the multiple cores of the CPU by using multi-threading.

We have looked into GPU based parallel processing (leveraging the SIMD architecture) as possible a method that should be able to reduce the required time for the ASIFT module, and thus reducing the overall run-time of our VMZ pipeline.

\subsubsection{ASIFT - Redundant operations removed}

The interface available for the IPOL-ASIFT consists of a single method that takes in two images and outputs the matching results consisting of matched feature-points for both images. We have looked into our specific use-case of ASIFT from VMZ, and determined that this single method can be broken down into two main methods:

- detect - detection operation of feature points of a single input image

- match - matching operation between features points of two images 
Breaking down into two separate methods helps with the redundant operation of feature-point detection that was being done previously, when the same image was being operated on multiple times. As feature-point detection is one of the compute intensive operations for ASIFT, this reduction of redundant operation impacts the run-time of ASIFT significantly.

The MATLAB workflow is able store the features extracted from an image by the ASIFT module, and can only run the detect method to generate the features for images being operated for the first time. This was the ASIFT module doesn't have to compute the feature points for the same image multiple times.

The matching operation takes in extracted features points for two images, and computes the matching operation by performing the regular SIFT matching operation. For the matching operation to work correctly, I had to ensure and validate that the exporting of the generated feature-points in the detect step and the importing of the feature-points for this match step are being done correctly. 


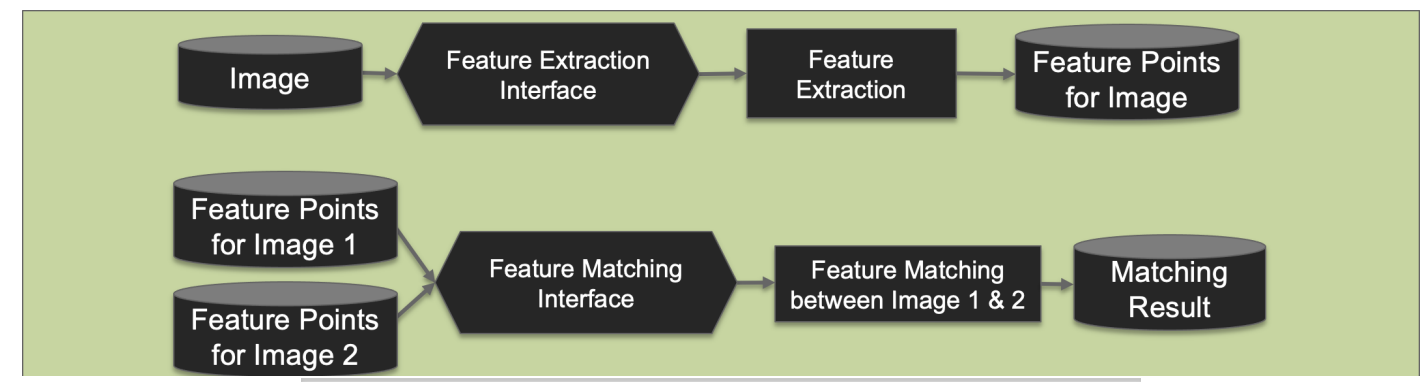

\footnotetext{
Improved ASIFT Calls (No Redundancy)

ASIFT_Detect("imageDir/img1.png", "kp_img1.txt", ...);

ASIFT_Detect("imageDir/img2.png", "kp_img2.txt", ...);

ASIFT_Detect("ImageDir/img3.png", "kp_img3.txt", ...);

ASIFT_Match("kp_img1.txt", "kp_img2.txt", ...);

match12 = fileread $($ matching12.txt"):

ASIFT_Match("kp_img2.txt", "kp_img3.txt", ...);

match23 $=$ fileread $($ "matching23.txt");
}

FIGURE 4.3: Block diagram showing the separated ASIFT pipeline for feature extraction and matching, which improved upon the previous pipeline by eliminating redundant feature-extraction operations, which can be seen from the the function calls shown below 


\subsubsection{MEX Interfacing}

Previously VMZ was using file input/output as the communication method, use specific filenames and directories were given as the input images which the $\mathrm{C}++$ library had to read from, and $\mathrm{C}++$ library writing out the result in a specific file, from which the MATLAB pipeline read the matching results. Any speed-up of this workflow is bottle-necked by the read-write speed of the hard drive, and any such bottleneck negatively affects the time required for any operations being done in the ASIFT module.

My work for improving the ASIFT module has removed this dependency on file read/write for communication, by leveraging the MEX file-function available for binding $\mathrm{C}++$ libraries with MATLAB through a custom written interface. For the ASIFT module which has it's redundant operations removed, I have created two MEX functions, one for the detect operation and another for the match operation.

The detect MEX function takes in an image read in MATLAB (in the Matrix datatype), and outputs keypoints - list, which is a feature-point matrix , and lengths, which is the length of featurepoints that is generated by each tilt configuration. This information is required to reconstruct the data-structure being used inside the ASIFT library for describing extracted features of an image. 
The match MEX function takes in as input, two keypoints list with corresponding lengths matrices, for the two images being matched. The output of this MEX function is a matrix containing the coordinates for the matched feature-points. 


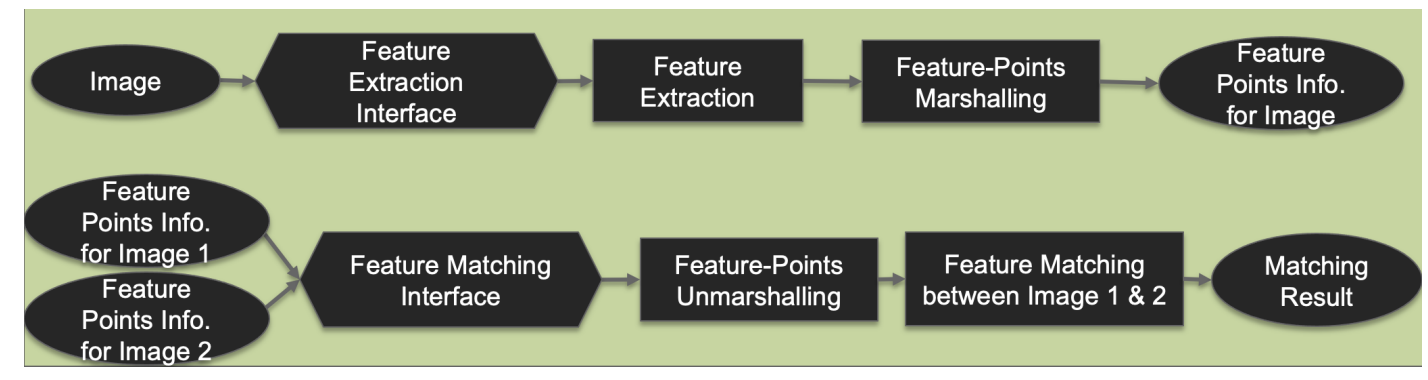

FIGURE 4.4: Block diagram showing the ASIFT improved pipeline with MEX interfacing for feature extraction and matching, removing all previous file read/write from disk. This also uses the separated interfaces for feature extraction and matching for removing redundant feature extraction operations 


\subsubsection{GPU Based Parallel Processing}

For leveraging the SIMD processing that is available though the use of GPU compute cores, we have parallelized ASIFT module, by using a GPU based implementation of SIFT. As described, ASIFT module internally generated multiple configurations for the given images by using the number of specified tilt. For each of such generated configuration, the regular SIFT method is then used to compute either the feature-points or to generate matches. This SIFT method that is found in the ASIFT module is a CPU based method. Our GPU based implementation replaces the underlying CPU based SIFT method by a GPU based SIFT method, we have used the CUDASIFT [22] implementation in this case.

\subsection{Proposed Blending Methods}

I've looked into multiple methods for the mosaic generation section of VMZ, which can be categorized into two broad types.

- Blending Methods to reduce illumination changes

- Proposed Illumination Matched blending 
As discussed earlier, in the paper [5], Replacement-Blending (Alpha blending with an alpha value of 1) coupled with a gradient/edge based condition is used, which results in a sharp mosaic. But it is very susceptible to illumination changes, as is seen from the mosaic result below.

\subsubsection{Blending Methods with Gradient condition}

I've investigated the gradient/edge based condition with 2 different blending techniques, such as Center-Weighing Feather Blending and Laplacian Pyramid Blending. Both types of blending are much capable than the replacement blending for merging of two images with different levels of illumination.

As the paper [5] clearly demonstrates that due to the deformable nature of the structure in the video sequences, the Gradientresponse Ratio (GRR) method helps selecting sharper images from a video sequence, which ultimately results in an overall sharper mosaic, I've incorporated the GRR condition with the blending techniques for generating the final mosaic result. As mentioned earlier, GRR is the ratio that is computed from the edge-response between the current frame and the current mosaic, if that ratio has a value of greater than 1 then it means that the structure in the current frame being processed is sharper than the mosaic, and vice-versa. The pseudo-code for this using 
GRR condition with the blending techniques are given below:

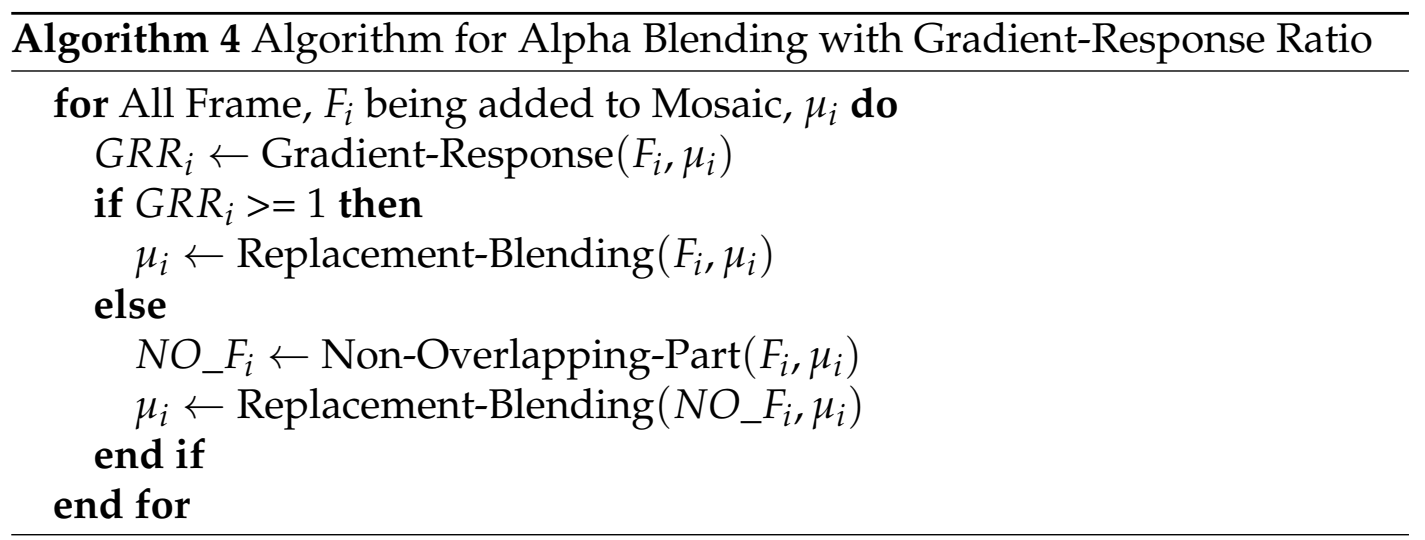

\section{Center-Weighing Feather Blending}

Traditional average blending actually uses alpha blending with the alpha value of 0.5. That doesn't always perform well, and can cause visible seams in the blended result, as shown below.

To overcome this visible seam in the blended result, I've used center-weighing feather blending, which uses the distance-transform of the two masks, for the two corresponding image, and computes a changing alpha value for each pixel of the overlapped part of the blended image, making the blending result much smoother.

\section{Laplacian Pyramid Blending}

The laplacian pyramid blending algorithm builds on top of the Gaussian pyramid approach, and uses the difference of gaussian result between subsequent levels of pyramid to compute the laplacian result for a certain level. Suppose, we have a task 
of blending two images with a mask describing which pixel to keep from the two images. For getting the blending result, we first compute the laplacian pyramid for both of the images, by computing the gaussian pyramid and then use those result to compute the laplacian pyramid. Then we use the provided mask to merge the laplacian pyramid for every level. And finally we collapse the merged laplacian pyramid to get the final blending result. 

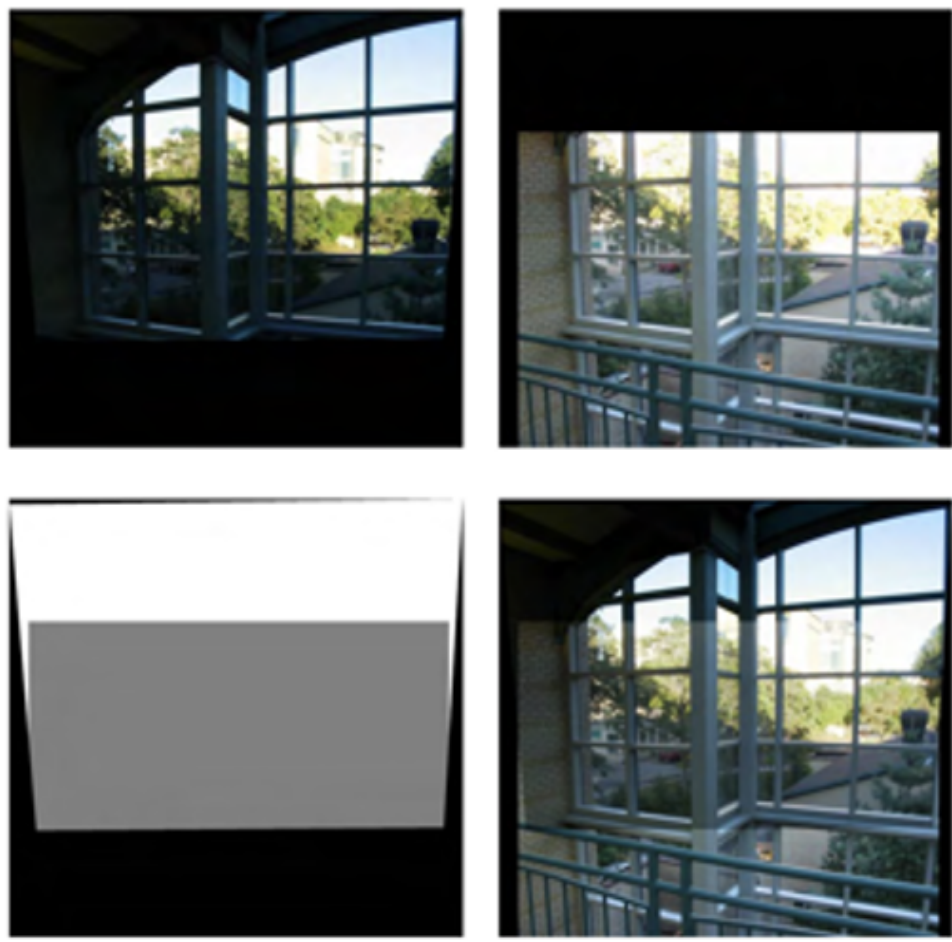

\section{Alpha $=.5$ in overlap region}
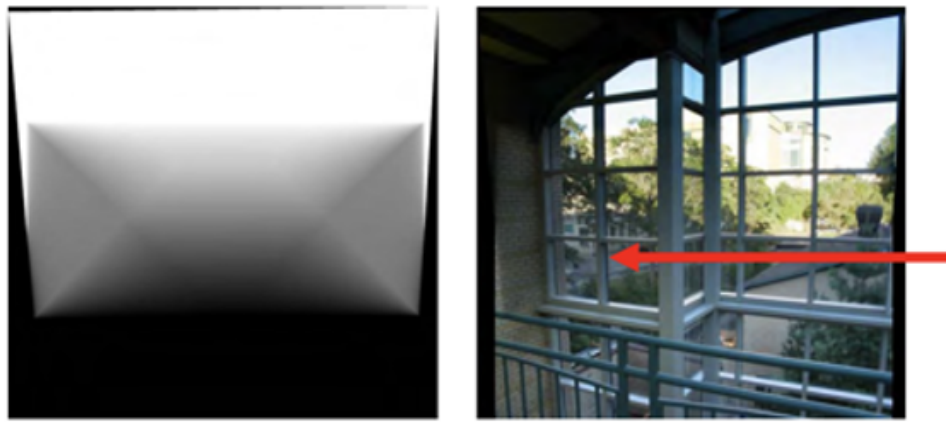

Alpha $=$ dtrans $1 /($ dtrans $1+d t r a n s 2)$

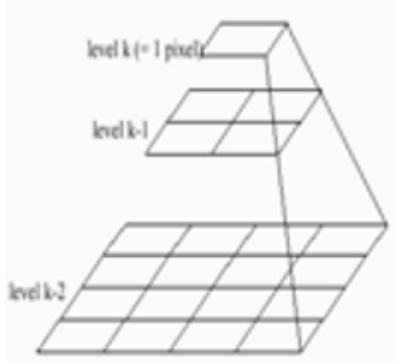

Left pyramid

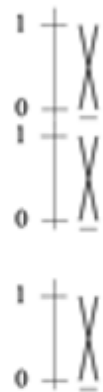

blend

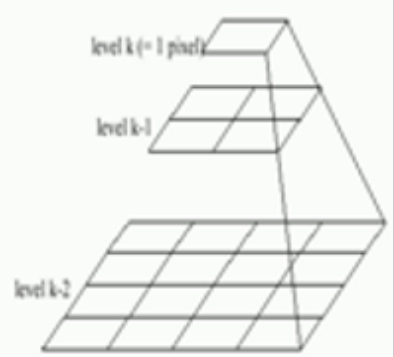

Right pyramid

FIGURE 4.5: Figure showing 3 types of blending. The first image (top 4 blocks) shows Alpha Blending with the alpha value set to 0.5 , resulting in an average blending. The second image shows the Feather Blending and the final image shows Laplacian Pyramid blending [19] 


\subsubsection{Illumination Matched Blending}

For the second type of method investigated for this project, I've devised an algorithm that tries to match the illumination, or the intensity in the case of gray level images, by computing the difference in the overlapped region of two subsequent image frames. After this illumination/intensity difference is computed for all of the overlapped pixels, the Shift-of-Intensity (SoI) value is computed from the mean of all intensity difference values. This Shift-of-Intensity (SoI) value is finally added to all of the pixels in the second frame to shift the illumination/intensity of that particular frame so that it better matches with the first frame.

$$
I D_{i}=\left(p_{i}-q_{i}\right)
$$

Here $p_{i}$ is the illumination/intensity value of $i$-th pixel from the first frame, and $q_{i}$ is the same value from the second frame.

$$
\begin{gathered}
S o I=\frac{\sum_{i=1}^{n} I D_{i}}{n} \\
C F=F+S o I
\end{gathered}
$$

For the equation 4.4 , shown above, the Changed-Frame (CF) is obtained by shifting the illumination/intensity value of the 
Frame (F) by adding the Shift-of-Intensity (SoI) value to all of the pixel values of the Frame $(F)$. The algorithm for this technique is described below:

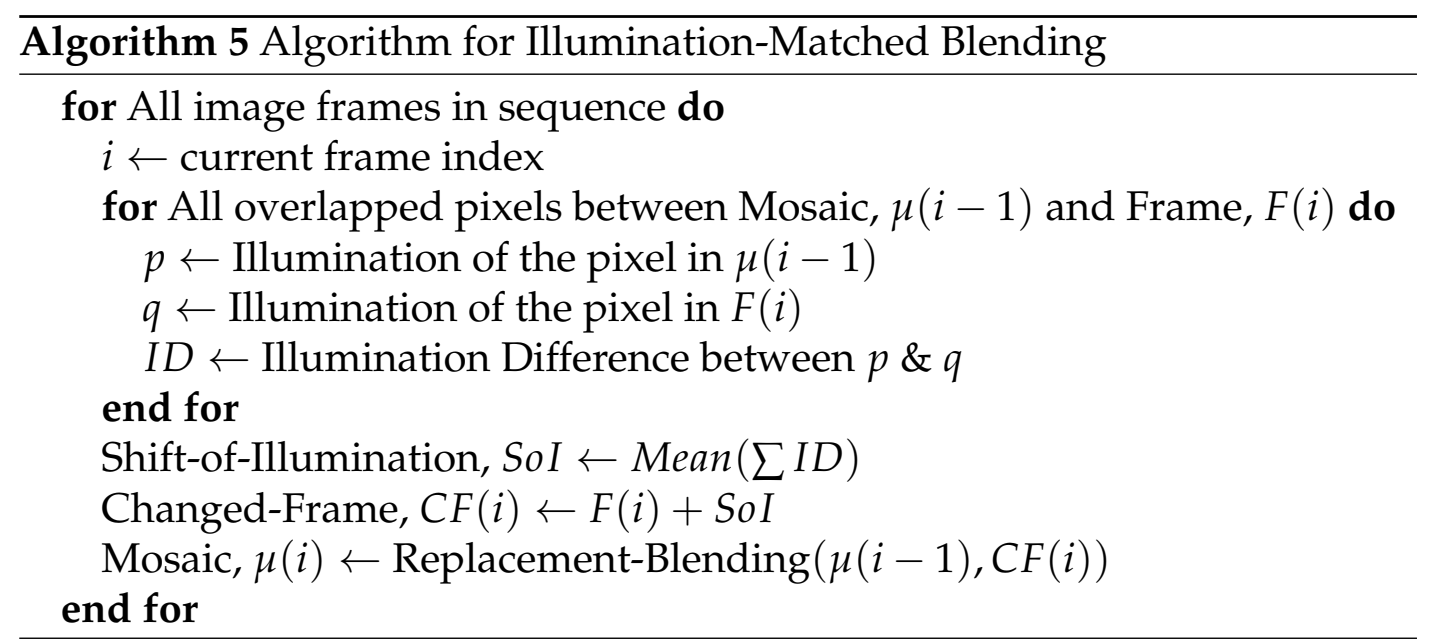

In the last step of the algorithm, I've used the replacement blending technique, as is used in the DNCC algorithm, though this step can also be using with other blending methods (and also the blending techniques described earlier)

\section{Proposed Illumination Difference Evaluation Metric}

Currently the metrics used to determine the resultant mosaics doesn't evaluate the illumination difference in the image frames which is used to generate the final mosaic. Only pixel level correctness and the structural-similarity are being evaluated using the MSE, PSNR and SSIM metrics, and no such evaluation of the illumination shift are being performed. 
For evaluating the resulting mosaics based on the the correctness of the mosaic and the structural similarity with the reference mosaic, I've used the the methods used in the DNCC paper, which are Mean-Square Error (MSE), Peak Signal-to-Noise Ratio (PSNR) and Structural-Similarity (SSIM). But the DNCC paper lacked an evaluation method that could measure the rapid illumination shifts between subsequent frames in the final mosaic. I've come up with an evaluation metric, Maximum Overlapped Intensity-Difference (MOID), which is elaborated below, given that we have $f$ number of image frames in the video sequence, $n$ number of overlapped pixels between two subsequent images:

$$
\begin{gathered}
I D_{i, j}=\left|p_{j}-q_{j}\right| \\
M I D_{i}=\frac{\sum_{j=1}^{n} I D_{i, j}}{n} \\
\text { MOID }=\max _{i=1}^{f} M I D_{i}
\end{gathered}
$$

The MOID will determine maximum shift of intensity between subsequent image frames of the resultant mosaic. 


\section{Chapter 5}

\section{Experimental Result}

\subsection{Improved Normalized Cross-Correlation}

For timing analysis of our methods with respect to the original MATLAB implementation, we selected three main factors. As stated previously, our dataset consists of 8131 image files, which contains a total of 933538 blocks. The factors we have chosen for our timing analysis are, Total-Time, the time required to calculate NCC matching for all of the 8131 images, Time-Per-Block, the average time required to calculate NCC matching over a single block, and Time-Per-Frame, the average time required to calculate NCC matching over a source image, searching for the template image in it. It can also be described as the average time required to calculate all of the blocks in a single image file.

For running our CPU based multi-threaded implementations, we have used a Intel(R) Xeon(R) CPU E5-2680 v4 @ 2.40GHz server with 14 cores with Hyper-Threading, so essentially 28 threads, and 252 GB of RAM. 
To experiment on the GPU, we tested with two different GPUs with varying amounts of Streaming Multiprocessors (SMs) and CUDA Cores. First was a GeForce GTX-1080 GPU. This has 20 SMs containing a total of 2560 CUDA Cores. Second was a Tesla V100, with 5120 CUDA Cores across 80 SMs. Because of increased interest in performing computation on the edge, we also tested on a Nvidia Jetson Xavier AGX. This unique device is very small and medium power. Its uses a GPU running NVIDIA Volta architecture. It contains 512 Nvidia CUDA Cores across 64 Tensor Cores.

We also tried our muti-threaded code on the Xavier. It contains $8 \mathrm{CPU}$ cores in a Carmel ARM v9.2 CPU which has a memory bandwidth of $137 \mathrm{~GB} / \mathrm{s}$.

\subsubsection{Results of Improved NCC}

For our two separate multi-threaded CPU implementation, in the Table 5.1, we have only shown the results for the implementation that used $\mathrm{C}++$ standard threading library. As we will also show, the timing differences between the two implementations, $\mathrm{C}++$ standard threads and OpenMP, tends to be minimal as the number of threads increases. We have also tested on threads numbering from 1 to 27 , but for the timing comparison, we have selected to show only the results of four instances, and included 
the timing results when running on a single thread, and when running on 7, 14 and 27 threads concurrently. From the selected set of timing for different number of threads, the actual timing trend for all of the threads can be easily seen. The top row of Figure 5.1 describes the timing comparison for all of the thread configurations.

It is evident from Table 5.1 that different threading libraries, like $\mathbf{C}++$ standard threads and OpenMP, for multi-threaded implementation, performs very similar and has little effect on the total time for running NCC computations as the number of threads increases. From here on, we will only focus on the multi-threaded implementation with the $\mathrm{C}++$ standard threads library.

Table 5.1 shows that switching to OpenCV, from MATLAB, gives some speedup straightaway, with no parallelism added. Further, it shows that we have a speedup as we increase the number of threads, but the speed increase does not increase linearly as the thread count increases. Finally, it shows that there is a speedup when using the GPU, but that speedup is more conservative than expected. This is because the NPP API does not take advantage of the full breadth of parallelism available, since it only parallelizes the given single NCC operation. The V100 
shows a much better improvement, likely due to better hardware specification. 


\begin{tabular}{||c|c|c|c|c||}
\hline \multicolumn{5}{||c}{ Timing comparison between the different methods } \\
\hline $\begin{array}{c}\text { NCC Computation } \\
\text { Method }\end{array}$ & $\begin{array}{c}\text { Total-Time } \\
\mathbf{( s )}\end{array}$ & $\begin{array}{c}\text { Time-Per-Block } \\
(\mathbf{m s})\end{array}$ & $\begin{array}{c}\text { Time-Per-Frame } \\
(\mathbf{m s})\end{array}$ & Speed-Up ${ }^{1}$ \\
\hline MATLAB & 12374.7 & 14.1 & 1522.0 & 1.0 \\
\hline C++ CPU - Threads = 1 & 3168.9 & 3.4 & 423.8 & 3.8 \\
OpenMP - Threads = 1 & 2791.3 & 3.0 & 343.3 & 4.4 \\
\hline C++ CPU - Threads = 7 & 822.3 & 0.9 & 99.7 & 15.0 \\
OpenMP - Threads = 7 & 810.7 & 0.9 & 99.7 & 15.3 \\
\hline C++ CPU - Threads = 14 & 497.1 & 0.5 & 60.4 & 24.9 \\
OpenMP - Threads = 14 & 489.5 & 0.5 & 60.2 & 25.4 \\
\hline C++ CPU - Threads = 27 & 313.7 & 0.3 & 39.2 & 39.5 \\
OpenMP - Threads = 27 & 319.2 & 0.3 & 39.3 & 38.8 \\
\hline Xavier CPU - Threads = 8 & 446.4 & 0.5 & 54.9 & 27.7 \\
\hline Xavier GPU & 1167.0 & 1.3 & 143.5 & 10.6 \\
\hline GTX-1080 GPU & 854.4 & 0.9 & 103.6 & 14.5 \\
\hline V-100 GPU & 152.5 & 0.2 & 18.7 & 81.1 \\
\hline
\end{tabular}

TABLE 5.1: This compares the performance at a high level, then at increasingly granular levels. Time-Per-Block for multi-threaded CPU implementation is the observed time calculated from the total time and number of blocks. *Speed-up is shown with respect to total time, but all columns show about the same results. The blue color uses OpenCV, the green color shows results for NPP to do GPU computation. Performance statistics reflect a real application workload with variable block sizes (majority of search blocks are 142x142), template block sizes (majority of search blocks are $40 \times 40$ ), an average of 120 blocks per frame, and 8132 frames. 

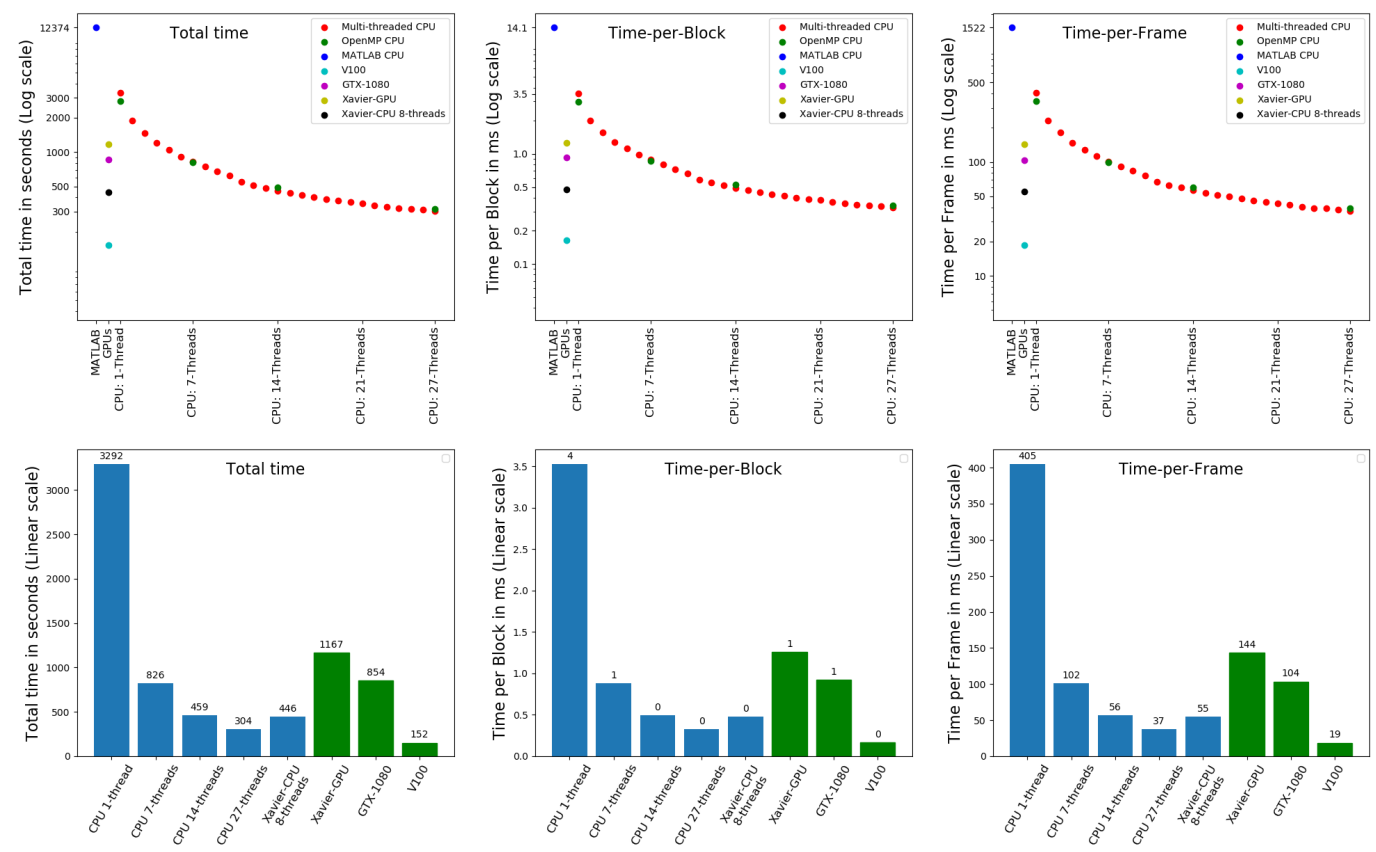

FIGURE 5.1: This is the timing comparison between all of our methods. The first column of plots are for the factor Total-Time (for all frames), second column of plots are for Time-Per-Block, and the third ones are for Time-Per-Frame. All of the plots in the first row are in Log scale for the Y (time) axis for including the MATLAB timing while keeping all of the other method timings distinguishable. The second row of plots excludes the MATLAB results, and are in Linear scale. In each of the plot, GPU results are shown under a single $X$-axis value, as well as the MATLAB result for the first row of plots, and the rest of the $X$-axis values, from 1 to 27 , denote the number of threads used for the CPU implementation. 


\subsubsection{Comparing the Output}

We computed the difference of the NCC results from the original MATLAB implementation with our multi-threaded CPU, and the GPU implementations using NPP. The correlation matrix result for the GPU implementation using NPP is very different from what we get from MATLAB and the multi-threaded CPU implementation using OpenCV, while the MATLAB result and the result from our multi-threaded implementation using OpenCV is quite similar. Below, we measured the peak points in the NCC correlation matrix from MATLAB and OpenCV, which is essentially the best point where the reference block matches in the search window. An exact match is defined where $(X-$ Peak, Y-Peak) is exactly same in MATLAB and OpenCV, and they have an euclidean distance of zero. In our tests, we have seen that around $\mathbf{9 4 . 9 \%}$ of blocks per frame has an exact match or zero euclidean distance. Including closely matching blockpairs with less than or equal to 4 pixels euclidean distance increases the percentage agreement between the MATLAB and multi-threaded OpenCV NCC peak results. In our experiment, we got $\mathbf{9 9 . 3 7 \%}$ of blocks with a close match or less than or equal to 4 pixel (radius) euclidean distance. Thus, only $\mathbf{0 . 6 3} \%$ of blocks have error greater than 4 pixels. In our experiment, we pick blocks (Structure Tensor (ST) feature points) which are far from 
image edges. The distance of any ST points should satisfy the following condition where edge_distance $S T$ refers to the minimum distance of a ST point from image edge, and $S W_{d}$ and temp $p_{d}$ stands for size of search block and template block respectively.

$$
\text { edge_distance }_{S T} \geq \frac{S W_{d}}{2}+\text { temp }_{d}
$$




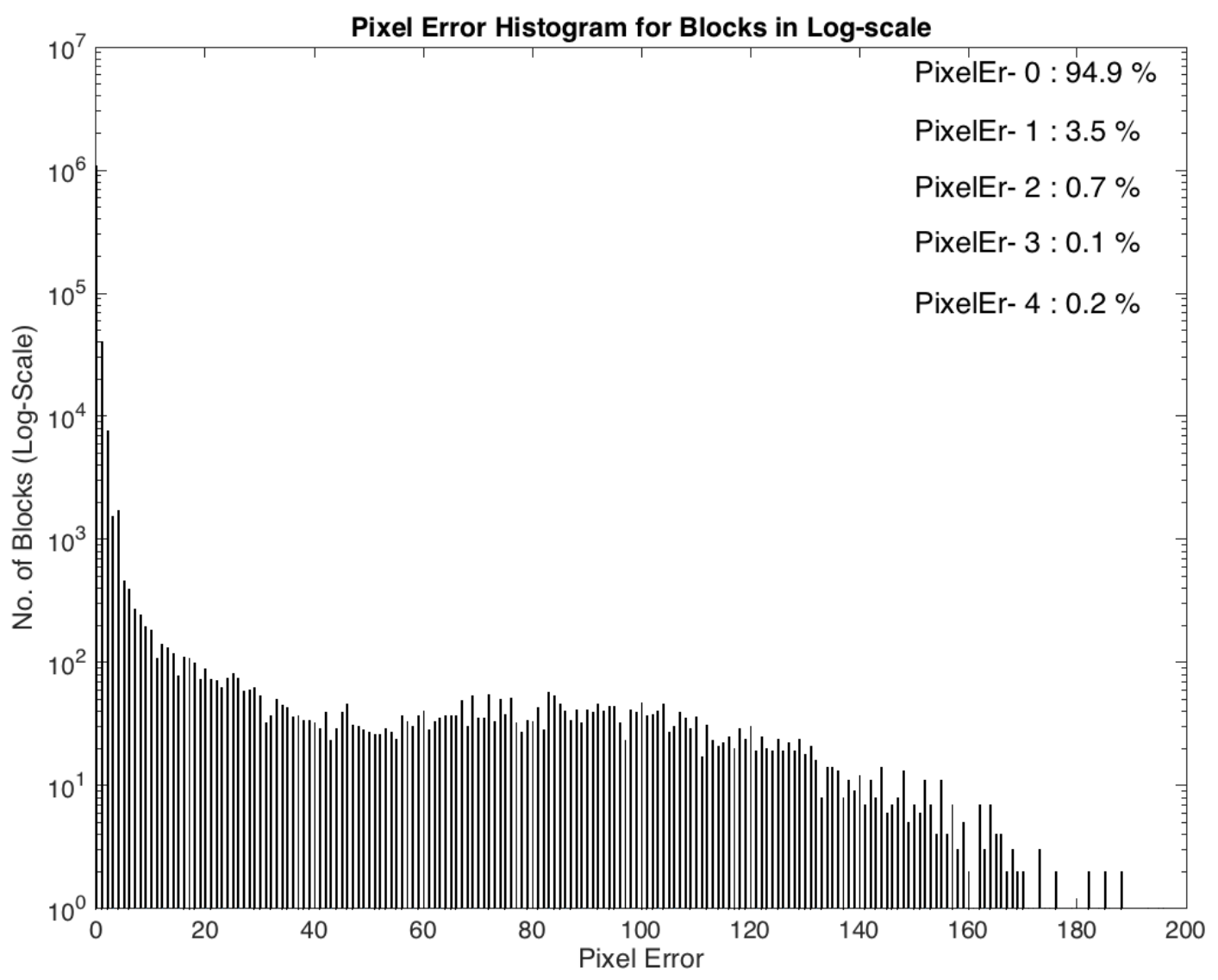

FIGURE 5.2: Histogram showing difference when comparing (X-Peak, Y-Peak)location of matched point- in MATLAB and our Multi-Threaded NCC implementation using OpenCV. X-axis represents pixel error between same block peak in MAT$\mathrm{LAB}$ and OpenCV where $\mathrm{y}$-axis stands for number of blocks with that error. From this graph, we can see that around $94.9 \%$ of blocks have an exact match, i.e., zero error between them. As the error increases, number of blocks drops significantly. For example, only $3.5 \%, 0.7 \%, 0.1 \%$ and $0.2 \%$ of blocks have pixel error of $1,2,3$ and 4 respectively. Only $0.63 \%$ of blocks have error greater than 4 pixels. 
For a comparison of the OpenCV and MATLAB results to the significantly non-matching results from NPP, it is best seen in Figure 5.3. Here it is clear that for these example images the correlation responses are quite different. NPP does not find values that are nearly as high except for where the peak is, which could actually be considered a more ideal (less noisy) NCC map. Still, because our implementation for mosaicing is based on the accuracy of MATLAB, we evaluate where the differences in the three algorithms comes from.

We have thoroughly checked the correlation matrix for all of our implementations and libraries used by NCC methods. And while OpenCV is open-source, and their implementation details can be seen directly from their code base, both MATLAB and NPP's NCC implementation details cannot be checked due to their closed-source nature. We have summarized that the difference in NCC correlation matrix and the peaks might be caused for multiple reasons.

1. For OpenCV's Cross-Correlation operation, using it's matchTemplate function, had 6 different methods for calculating the correlation matrix. We have selected the method 'CV_TM_CCOEFF_NORMED', which is one of the normalized methods available, and the equation for this method is given in the documentation. Internally, this method uses an Integral-Image and Fourier-Transformation 
for optimization. But for MATLAB's and NPP's NCC operation, using it's normxcorr 2 and 'nppiCrossCorrValid_NormLevel_32f_C1R', respectively, they don't give any implementation details, and work as a black-box. So we could not be sure of the exact method, implementation and optimization techniques being used in it's case.

2. The peak finding algorithm used for MATLAB after calculating the correlation matrix was selecting the peak in a columnwise order, where in OpenCV/C++ it was finding the peak in the correlation matrix in a row-wise order. NPP chooses the tied max at the top left of the image. This only creates a mismatch if there are multiple peaks in the correlation matrix with the same maximum value.

3. Checking the correlation matrix from both OpenCV/C++ and MATLAB's NCC computation, it was evident that the size of the correlation matrix for MATLAB is larger in both dimension than the correlation matrix for OpenCV. We found out that this change initially occurs in the Search-Window, where the normxcorr 2 function of MATLAB automatically applies a padding so that it can also calculate the correlation in the border regions of the search-window. And this padding in the search-window causes the computed correlation matrix to be larger in size as well. The matchTemplate function of OpenCV doesn't apply any 
kind of padding while performing the NCC calculation. The dimensions for correlation-matrix can be computed from the search-window and template sizes, where $C M_{d}$ is the CorrelationMatrix size, $S W_{d}$ is the Search-Window size, and Temp $p_{d}$ is the template block size (assuming square blocks),

$$
C M_{d}=\left(S W_{d}-\operatorname{Temp}_{d}\right)+1
$$

This Correlation-Matrix size computation is same for both MATLAB and OpenCV. But the Search-Window (SW) size for MATLAB differs from OpenCV as the additional padding in applied on it. For MATLAB, the Search-Window size increases by following this equation, where $S W P_{d}$ is the Search-Window size with the added padding,

$$
S W P_{d}=S W_{d}+\left(2 *\left(\operatorname{Temp}_{d}-1\right)\right)
$$

It can be seen from Figure 5.3 that the correlation-matrix for MATLAB is larger than the other two methods and contains border effects, which is the reason for the zero-padding added to the Search-Window. There are different variations of the NPP function we used that use "Full" mode, which allow us control over when 0 padding is used - thus it is not an issue for NPP. 


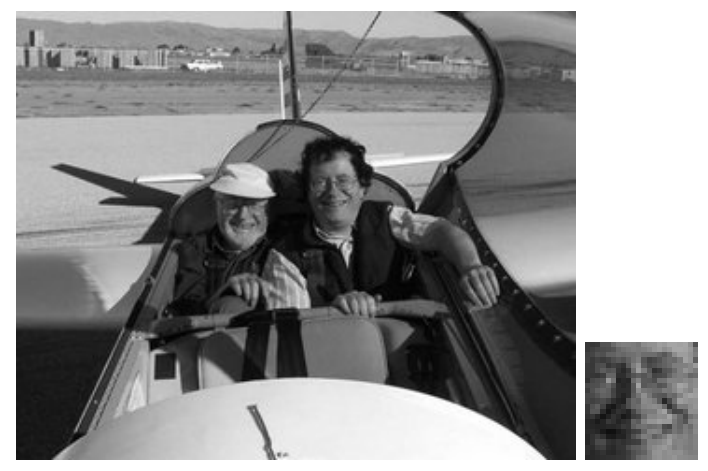

(a) Test source image and template
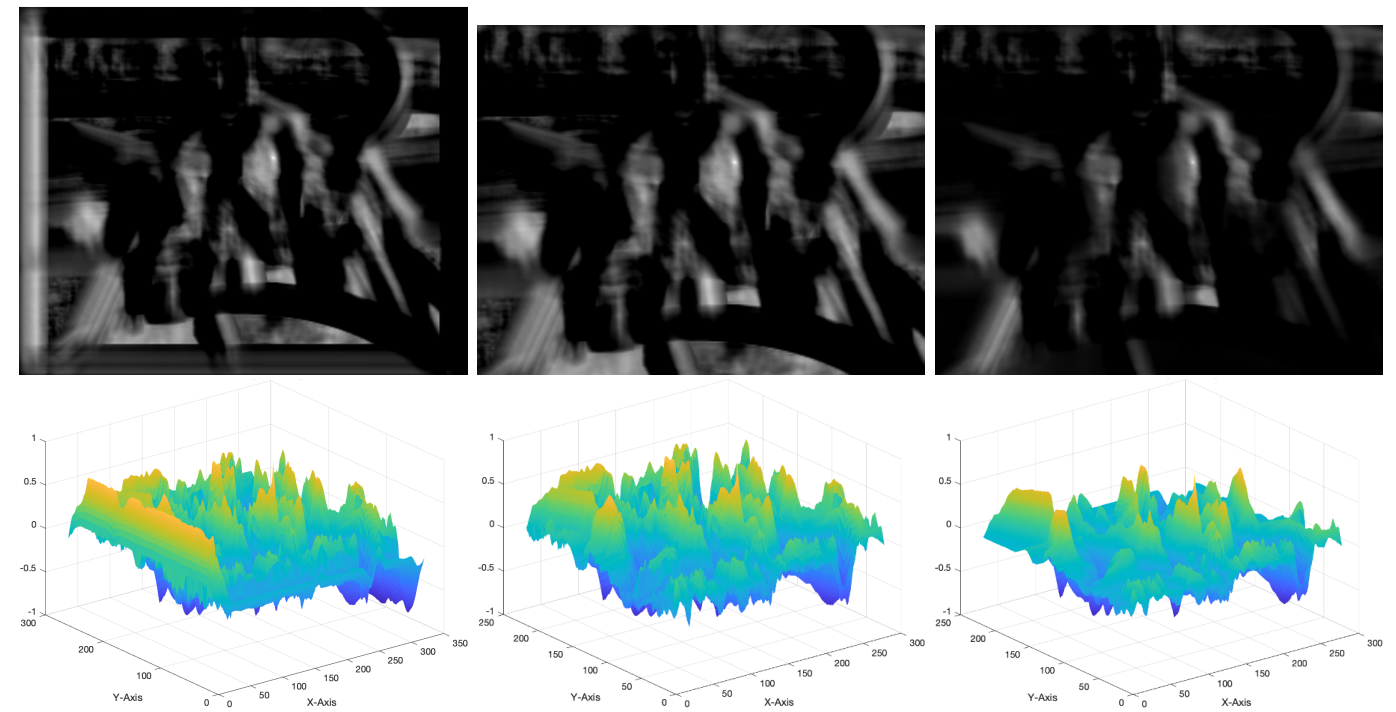

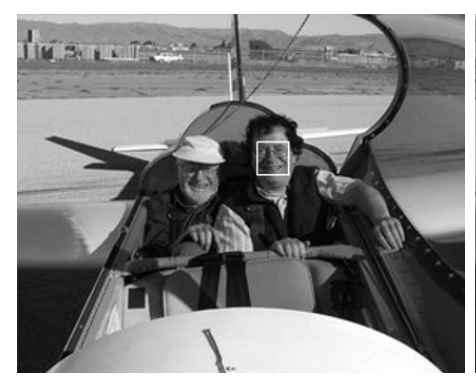

(b) MATLAB

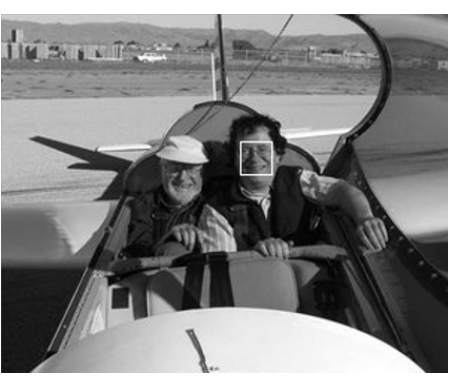

(c) OpenCV-C++

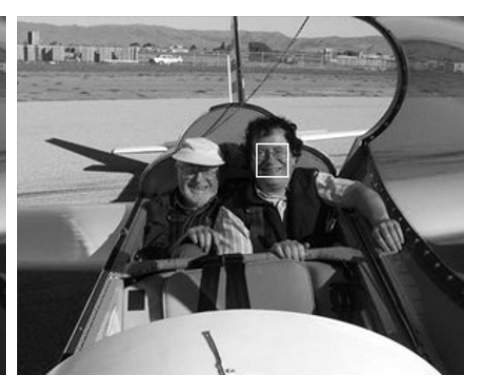

(d) NPP (GPU)

FIGURE 5.3: This figure illustrates the correlation-matrix and peak comparison between all of our methods. The first row contains the source image and the template, which is to be searched within the source image. The next rows show the correlation matrix and template matched to the source image for each method. The MATLAB result in Row 2 includes the padding which is the default behavior. 
4. As for the difference between OpenCV/MATLAB and the NPP API used, we have found a few major contributing factors. First, precision and some normalization seem to be responsible for reducing the size and number of peaks, this is evident from the correlation matrices and corresponding 3D response maps shown in Figure 5.3. Because the implementation is hidden from us, we cannot be sure, but it seems to be a regional normalization. We found this to be proven by NCC performed with templates taken from the image itself. The peaks were never exactly one, likely because they were averaged with the scores around them. 


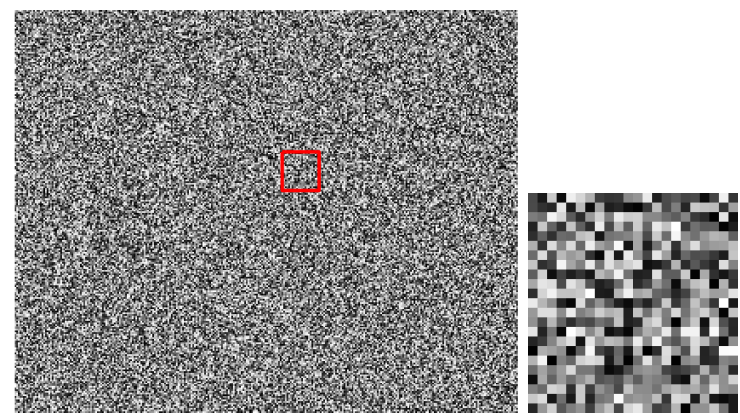

(a) Template marked in Source (b) Template

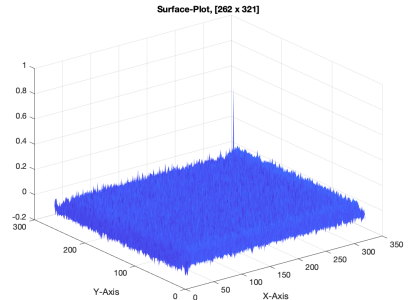

(c) OpenCV Correlation Matrix (SW Padded)

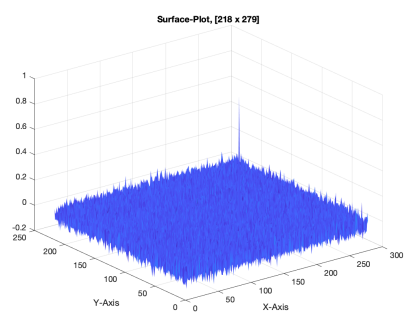

(f) OpenCV Correlation Matrix

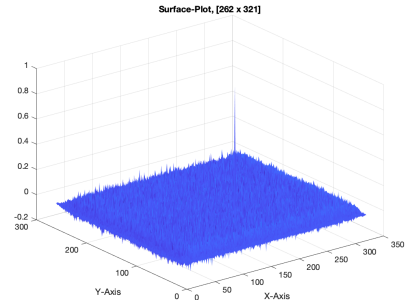

(d) NPP Correlation Matrix (SW Padded)

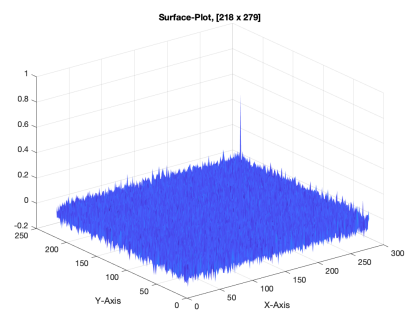

(g) NPP Correlation Matrix

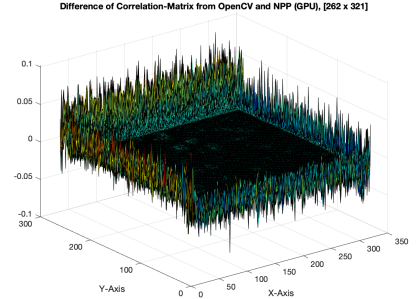

(e) Difference (SW padded)

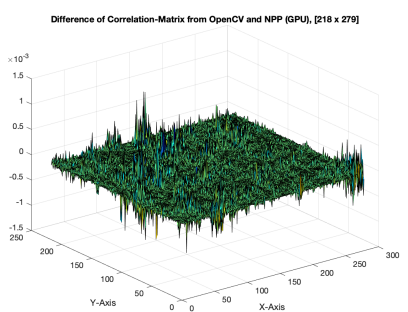

(h) Difference (no SW padded)

FIGURE 5.4: For demonstrating the differences in the results for NPP from both MATLAB and Multi-Threaded CPU implementation using OpenCV, we have generated a random image as a source image of dimension (300x240), within which we will search our template-patch, and selected a patch from it as the template of dimension (22x23). For computing the difference map, we have taken the correlation matrix from both OpenCV and NPP, and for the first image we can see that most of the differences are in the zero padded region, which is homogeneous, and in the rest of the image where variance is high, the difference is very small. This is also apparent from the second difference map, as there is no homogeneous region due to no padding, all of the differences are in the $10^{-3}$ region. 


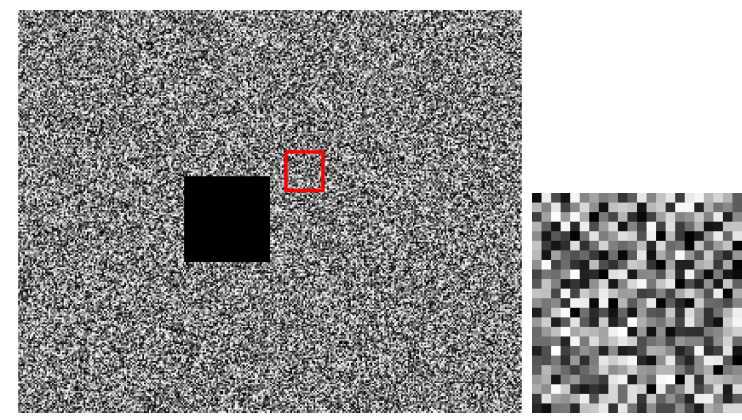

(a) Template marked in Source (b) Template

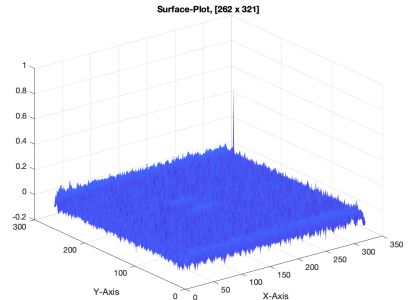

(c) OpenCV Correlation Matrix (SW Padded)

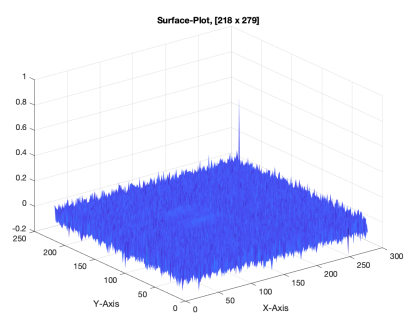

(f) OpenCV Correlation Matrix

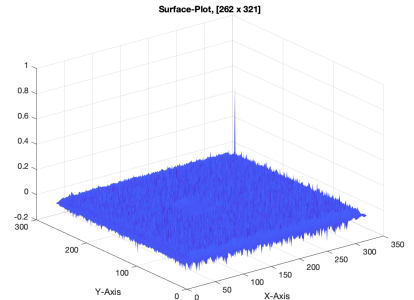

(d) NPP Correlation Matrix (SW Padded)

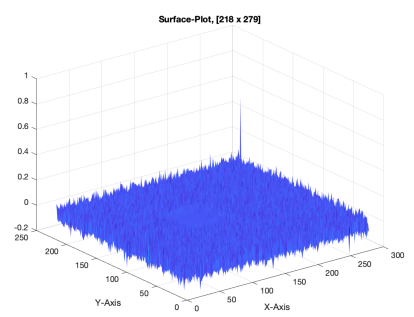

(g) NPP Correlation Matrix

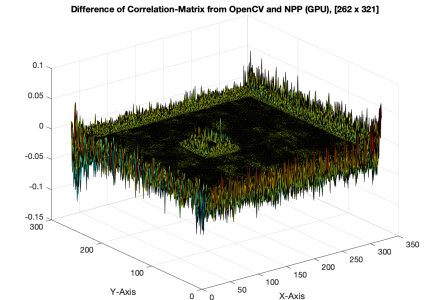

(e) Difference (SW padded)

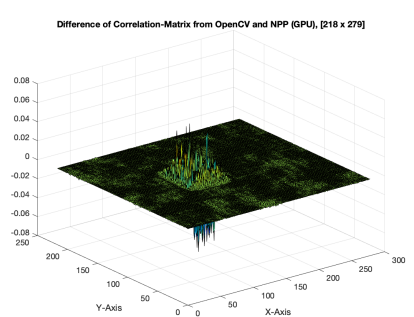

(h) Difference (no SW padded)

FIGURE 5.5: We have also generated a random image with a flat patch (homogeneous) inside it as a source image of dimension (300x240), within which we will search our template-patch, and selected a patch from it as the template of dimension (22x23). Similar to the previous figure 5.4, for the difference map, we have taken the correlation matrix from both OpenCV and NPP, and for the first image we can see the differences are in the zero padded region as well as in the patch with flat region, which is homogeneous, and in the rest of the image where variance is high, the difference is very small. This is also apparent from the second difference map, as the only homogeneous region there is the flat patch and the difference is high in that region, and for all other region, the differences are in the $10^{-3}$ region. 
5. The more significant difference is a lack of instability handling in NPP. Because the NCC algorithm divides by the variance, there can be issues when a homogeneous region is taken as the source or template, this is illustrated in Figure 5.4 and Figure 5.5. Divide by zero or divide by very small amounts causes overflow or other related problems in the output. This can be handled by discarding bad values or by regularizing, adding a constant to the numerator and denominator. OpenCV and MATLAB implementations handle these cases, while NPP does not. This makes it unreliable, as in natural imagery we will use many homogeneous regions and the issue cannot be ignored. Luckily, the OpenCV GPU matchTemplate algorithm handles these cases, so we still have a GPU option. However, this API has poor performance shown in experiments for Section 5.1.3.

From the speedup results, we can conclude that parallelization results in a significant speed improvement for NCC. Parallelizing across all the template matching needed for a pair of frames, as in the CPU multi-threaded implementation, brought us up to a 40x improvement, although this requires a large number of threads. The performance increase does not scale linearly with threads, as it would seem some overhead prevents this.

Parallelizing the NCC operation itself, as in the GPU implementation, also saw great improvements. Per NCC operation, 
we saw a 10-80x improvement depending on the hardware. It is much harder to draw any conclusions about what this improvement scales with, as we did not have enough GPUs of similar hardware to test with.

We also saw good performance on the Jetson Xavier AGX, where there is potential to pre-calculate NCC scores for mosaicing on the edge.

Furthermore, the data in Figure 5.2 shows that our NCC implementations are not pixel perfect identical, and there are many possible reasons for that still being investigated, as evident from the above discussion. 


\begin{tabular}{|c|c|c|c|c|c|c|}
\hline \multirow{2}{*}{ Method } & \multirow{2}{*}{ Measure } & \multicolumn{5}{|c|}{ Template Size } \\
\hline & & $(16 \times 16)$ & $(32 \times 32)$ & $(64 \times 64)$ & $(128 \times 128)$ & $(256 \times 256)$ \\
\hline \multirow{2}{*}{ MATLAB } & Time-Per-Block (ms) & 344.8 & 441.7 & 548.4 & 500.8 & 577.4 \\
\hline & Speedup & 1 & 1 & 1 & 1 & 1 \\
\hline $\mathrm{C}++/$ OpenCV & Time-Per-Block (ms) & 229.9 & 230.0 & 272.1 & 274.5 & 245.6 \\
\hline Thread-1 & Speedup & 1.5 & 1.9 & 2.0 & 1.8 & 2.4 \\
\hline $\mathrm{C}++/$ OpenCV & Time-Per-Block (ms) & 41.6 & 41.8 & 48.2 & 48.4 & 43.0 \\
\hline Threads-7 & Speedup & 8.3 & 10.6 & 11.4 & 10.3 & 13.4 \\
\hline $\mathrm{C}++/$ OpenCV & Time-Per-Block (ms) & 22.3 & 22.4 & 26.3 & 27.2 & 23.7 \\
\hline Threads-14 & Speedup & 15.5 & 19.7 & 20.9 & 18.4 & 24.4 \\
\hline $\mathrm{C}++/$ OpenCV & Time-Per-Block (ms) & 12.8 & 12.4 & 14.4 & 14.5 & 13.8 \\
\hline Threads-27 & Speedup & 26.9 & 35.6 & 38.1 & 34.5 & 41.8 \\
\hline $\mathrm{C}++/$ OpenCV & Time-Per-Block (ms) & 128.1 & 122.6 & 124.8 & 151.5 & 165.5 \\
\hline Xavier CPU 8 Thread & Speedup & 2.6 & 3.6 & 4.4 & 3.3 & 3.5 \\
\hline Xavier GPU NPP & $\begin{array}{l}\text { Time-Per-Block (ms) } \\
\text { Speedup }\end{array}$ & $\begin{array}{l}23.8 \\
14.5\end{array}$ & $\begin{array}{l}76.6 \\
5.8\end{array}$ & $\begin{array}{l}293.9 \\
1.9\end{array}$ & $\begin{array}{l}1098.3 \\
0.5\end{array}$ & $\begin{array}{l}3836.3 \\
0.2\end{array}$ \\
\hline GTX-1080 GPU NPP & $\begin{array}{l}\text { Time-Per-Block (ms) } \\
\text { Speedup }\end{array}$ & $\begin{array}{l}24.3 \\
14.2\end{array}$ & $\begin{array}{l}93.6 \\
4.7\end{array}$ & $\begin{array}{l}362.4 \\
1.5\end{array}$ & $\begin{array}{l}1382.3 \\
0.4\end{array}$ & $\begin{array}{l}4845.7 \\
0.1\end{array}$ \\
\hline V-100 GPU NPP & $\begin{array}{l}\text { Time-Per-Block (ms) } \\
\text { Speedup }\end{array}$ & $\begin{array}{l}2.1 \\
164.2\end{array}$ & $\begin{array}{l}77.7 \\
57.4\end{array}$ & $\begin{array}{l}29.4 \\
18.7\end{array}$ & $\begin{array}{l}109.2 \\
4.6\end{array}$ & $\begin{array}{l}382.0 \\
1.5\end{array}$ \\
\hline $\begin{array}{l}\text { Xavier GPU } \\
\text { OpenCV CUDA NCC }\end{array}$ & $\begin{array}{l}\text { Time-Per-Block (ms) } \\
\text { Speedup }\end{array}$ & $\begin{array}{l}205.3 \\
1.7\end{array}$ & $\begin{array}{l}198.8 \\
2.2\end{array}$ & $\begin{array}{l}197.4 \\
2.8\end{array}$ & $\begin{array}{l}197.7 \\
2.5\end{array}$ & $\begin{array}{l}197.5 \\
2.9\end{array}$ \\
\hline
\end{tabular}

TABLE 5.2: Results for our randomly generated 2048x2048 image for different sized template blocks. Workload is based on 100 random template blocks of five sizes, with the whole image as the search window, averaged over 400 million NCC computations. 


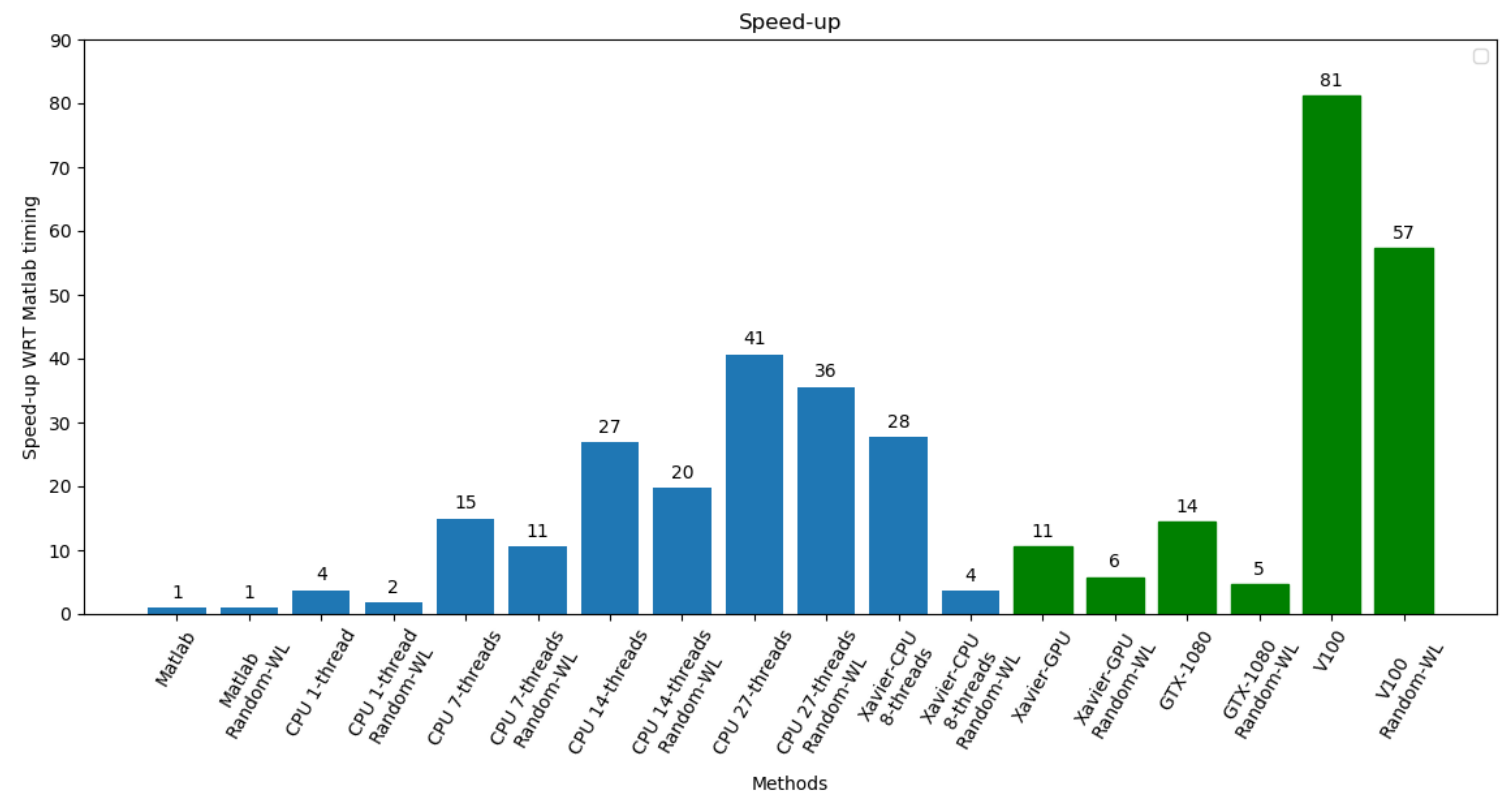

FIGURE 5.6: Speed-Up shown for two different workloads, 5.1 and 5.2, for our different methods with respect to the original MATLAB implementation. Five instances of CPU thread configurations are shown here marked as blue bars, and the green bars denote the speed-up for the three different GPUs. 


\subsubsection{Further Results}

Due to our findings showing that the methods only have the same output with a completely random image, we also compiled speedup results with this style of input. Since we were generating random input, we were now able to characterize the timing performance with respect to the size of template blocks. Table 5.2 gives the results and new speedup for five different selected template sizes, 16x16, 32×32, 64×64, 128x128 and 256x256, respectively.

First, we show that the speedups reported previously are still accurate with exactly matching output. While these speedups shown for the multi-threaded CPU C++ implementation in both tables are slightly slower, this can be attributed to the significantly larger source image (2048x2048), and the resulting much higher number of computations required.

The GPU implementation in Table 5.2 shows a higher performance than with the VIRAT data from Table 5.1, but it slows down significantly with larger template sizes like 256x256, shown with Table 5.2. We have a few hypotheses for why, but we cannot be sure due to the closed off nature of the NPP API's. One possible reason is an increased number of bank conflicts as the amount of data grows unwieldly. This is especially likely, as the search window size in this case is very large at 2048x2048. With 
the VIRAT data, our search window sizes were 10-16x smaller. We found that GPU utilization rises to $100 \%$ at template size 64 , showing that their algorithm correctly scales as much as possible with number of threads while it must be dealing with the size of the data, causing the slowdown. Also possible is an algorithm difference where NPP does not implement the integral image or the Fourier transform denominator.

This shows that it may be best to scale NCC by allowing each thread to handle an entire NCC calculation, as was done with our CPU multi-threading. It seems to scale better and with more available hardware.

We also had the opportunity to test the CUDA enabled OpenCV matchTemplate function. The correlation matrix output is exactly identical to the CPU version, but it parallelizes the NCC computation like NPP does. Table 5.2 shows that it is not similar in implementation to NPP at all, however. It shows that the speedup actually increases with template size, the opposite of NPP. It is also significantly slower than most threaded CPU implementations. Thus, is has some merit as a slower, more scalable and numerically stable version of NCC for the GPU. 


\subsection{Improved Structure-Tensor Module}

\subsubsection{Dataset Used}

I've used images from the VIRAT dataset tape2_8 for comparing the results for Structure Tensor features. The tape2_8 of the VIRAT dataset contains 9232 images, and for calculating the speed-up with the MATLAB implementation of Structure Tensor, I've used the first 100 images from the image set.

\subsubsection{Hardware Used}

For running the VMZ MATLAB implementation of Structure Tensor, I have used a Intel(R) Xeon(R) CPU E5-2680 v4 @ 2.40GHz server with 14 cores with Hyper-Threading, which essentially has 28 threads, and 252 GB of RAM.

To experiment the Structure Tensor GPU implementation, I've used a Tesla V100, with 5120 CUDA Cores across 80 Streaming Multiprocessors.

\subsubsection{Results for Improved ST}

The MATLAB implementation was run with the first 100 image frames of the tape2_8 for 50 times, and the average time was taken for the processing of 100 frames. The same dataset with the same load was run with the GPU implementation, so that a 


\begin{tabular}{|c|c|c|c|}
\hline Structure Tensor Versions & Image size & Execution time in ms & Speed-up \\
\hline \hline MATLAB & $640 \times 480$ & 7349.44 & 1.0 \\
\hline GPU & $640 \times 480$ & 72.10 & 101.93 \\
\hline
\end{tabular}

TABLE 5.3: Time required and speed-up for Structure Tensor

direct comparison can be performed over the two implementations. The image read time was removed from both of the tests, to ensure that we are only comparing the computation time required for the different Structure Tensor implementations.

From the table 5.3, we can see that for the same workload, the GPU implementation of Structure Tensor is almost 100x faster than the MATLAB implementation. This is due to the image processing operations of computing the difference between subsequent pixels, $I x, I y$, can be done in parallel, without any dependency on other operations, using the GPU's inherent Single Instruction Multiple Data pipeline.

The initial Structure Tensor implementation that is being used for VB3D only retains the coordinates for each maximal feature point and the scale (sigma value) for that particular feature. It doesn't retain the response values, which is the eigenvalue computed from the Structure Tensor matrix, in it's internal datastructure. Which is the main reason why selecting the top or best features among all of the computed Structure Tensor feature is not readily possible with the library. 

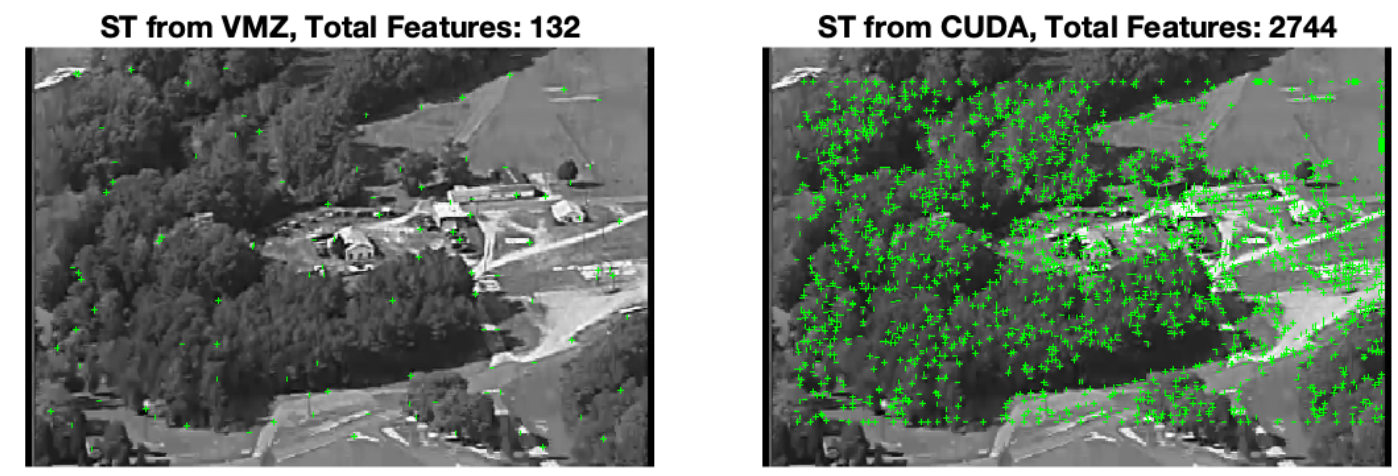

(a) ST features from MATLAB (left) and GPU (right) for Frame_000124
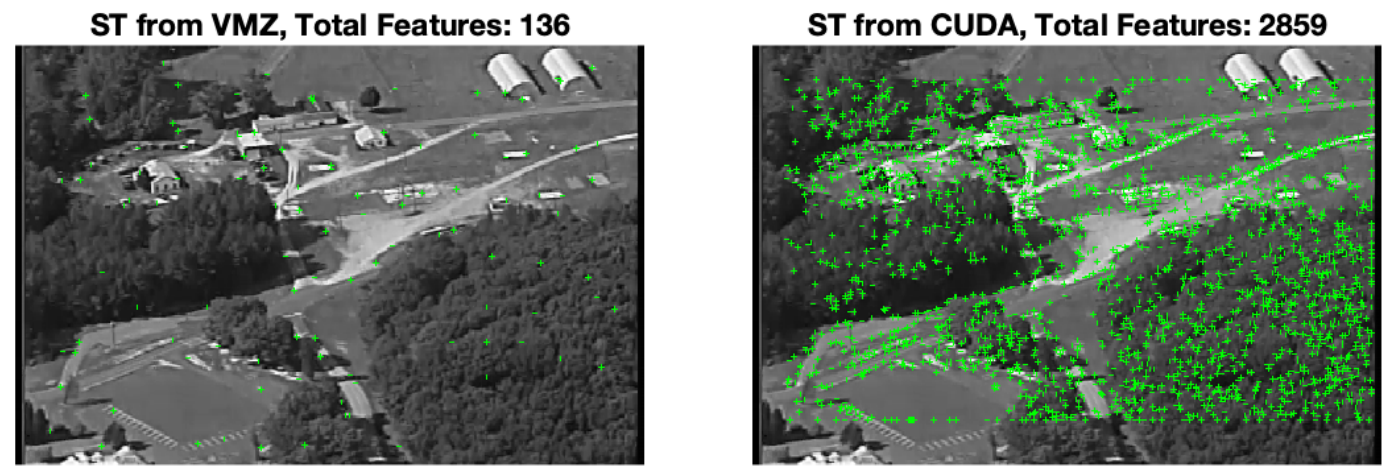

(b) ST features from MATLAB (left) and GPU (right) for Frame_000164

FIGURE 5.7: ST features comparison between MATLAB and GPU implementation without any selection within the feature points list 
For keeping the matching pipeline fast, we need to keep the total number of feature points constrained by some way. Otherwise, as can be seen from 5.7, more than 2700 features are extracted for each images from the GPU implementation, and matching such a high number of features between both of the images will take much longer time, and the speed-up gain from the GPU implementation will be negatively impacted by this.

For constraining the number of feature points, I have retained the eigenvalue of the Structure Tensor matrix for each of the maximal feature points by changing the internal data structure. After this response value is being retained, along side the feature point coordinate and the scale (sigma value), we can perform a selection process by sorting the feature points by their response value, and select only the top $K$ number of features that we most interested in. By limiting the number of feature points in such way, we can keep the internal data-structure small, and the matching time short.

From figure 5.8, we can see that selecting the top 150 features based on the response values compare with the Structure Tensor features extracted from the MATLAB pipeline. 

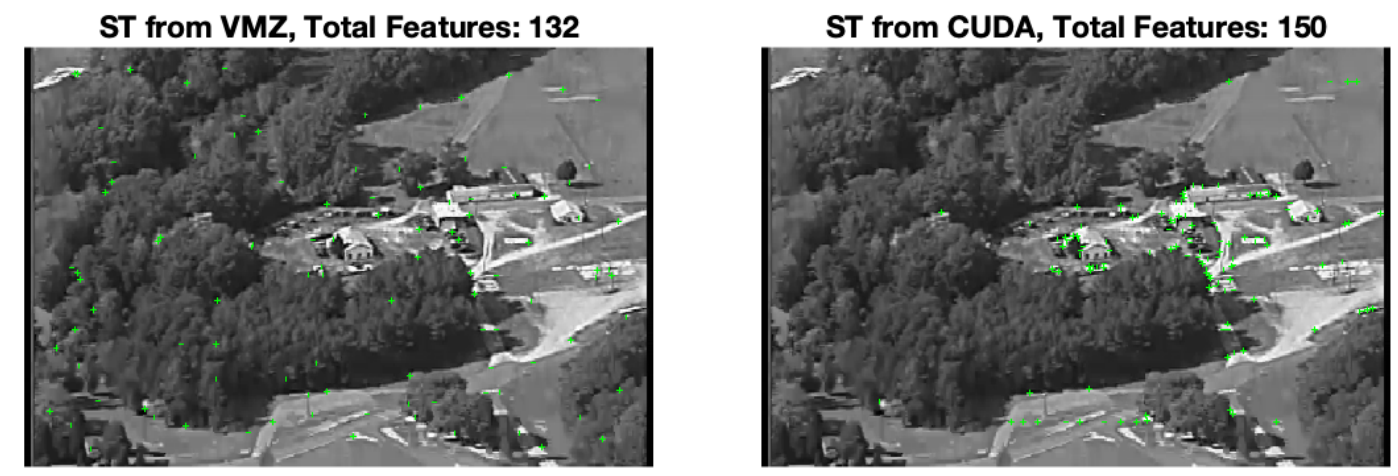

(a) ST features from MATLAB (left) and GPU (right) for Frame_000124
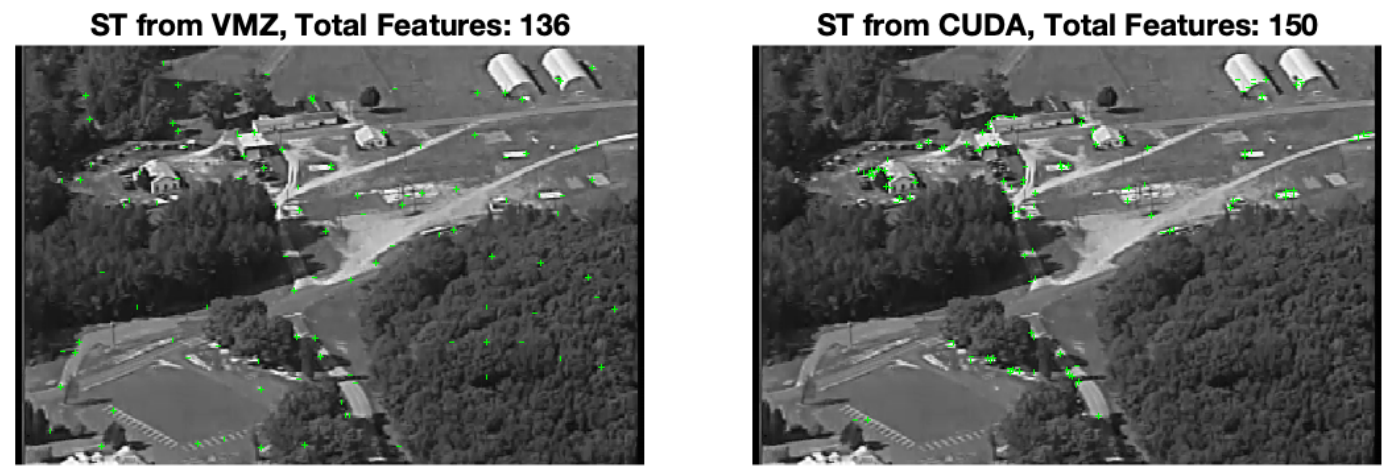

(b) ST features from MATLAB (left) and GPU (right) for Frame_000164

FIGURE 5.8: ST features comparison between MATLAB and GPU method, with top150 features selected from the GPU implementation 
In figure 5.8, we also see that the extracted feature points for the different implementations are not similar, and specially for the MATLAB implementations, a lot of feature points are actually selected in homogeneous regions, which is to say that those features will be especially difficult to match, as feature points in a homogeneous region will contain very similar feature descriptors. This is being caused by how the MATLAB implementation is selecting the feature points from the maximal Structure Tensor points. In the MATLAB implementation, the feature points are selected in a block by block manner, where for every block of a fixed size in the image, a predetermined number of feature points are selected. This causes a more distributed selection of feature points in the image, but also causes feature points to be selected from homogeneous regions, there the actual response value for those particular feature points are much lower than the actual top- $\mathrm{K}$ feature points found in the whole image.

\subsection{Improved Affine-Invariant SIFT}

\subsubsection{Dataset Used}

I've used images from the VIRAT dataset tape2_8 for comparing the results for the different Affine-Invariant SIFT modules. The tape2_8 of the VIRAT dataset contains 9232 images, and for 
calculating the speed-up between the original IPOL-ASIFT with file-input/output with the modified ASIFT with MEX interfacing, I've used the first 20 images from the image set.

\subsubsection{Hardware Used}

For running the VMZ MATLAB implementation of Affine-Invariant SIFT, I have used a Intel(R) Xeon(R) CPU E5-2680 v4 @ 2.40GHz server with 14 cores with Hyper-Threading, which essentially has 28 threads, and 252 GB of RAM.

\subsubsection{Results for Improved ASIFT}

I have performed improvements over the original implementation of the IPOL ASIFT implementation in two steps, as discussed previous in Methods section of this thesis. The first stage of modification contains the splitting of the single interface that was present in the original IPOL-ASIFT implementation into two separate interfaces, one for performing feature point detection operation on a single image, and another for performing match operation on the extracted feature points from a pair of images.

For the speed-up, I've computed the run-time of the two ASIFT modifications over the original implementation with two workflows. For the first one, I've computed the time required for 


\begin{tabular}{|c|c|c|c|}
\hline ASIFT Versions & Image size & Total time (in sec) & Speed-up \\
\hline \hline IPOL-ASIFT & $640 \times 480$ & 6246.81 & 1.00 \\
\hline ASIFT-RR & $640 \times 480$ & 1556.91 & 4.01 \\
\hline ASIFT-MEX & $640 \times 480$ & 945.68 & 6.62 \\
\hline
\end{tabular}

TABLE 5.4: Time required and speed-up for ASIFT implementation over VMZ pipeline

ASIFT operation while the VMZ pipeline is running over the VIRAT dataset tape2_8 for the three different versions of ASIFT. This is a more real-life scenario, where we can see how the modification for removing the redundant feature point detect operation actually plays a role in speed-up from the original implementation of ASIFT.

For the second workflow, I've timed the two modified ASIFT versions over a two images selected from the tape $2 \_8$ of the VIRAT dataset, and performed the feature point detect operation 10 times for each of the images and each pair of detect operation is followed by a match operation. In total, this workflow has 20 iterations of feature point detect operation and 10 iterations of match operation between the pair of images.

\subsubsection{GPU Implementation Results}

The GPU Implementation of ASIFT replaces the CPU based SIFT operation for detection and matching within the algorithm with a GPU based SIFT operation, which is CUDASIFT [add-referencehere]. CUDASIFT is a variant of the traditional SIFT algorithm, 


\begin{tabular}{|c|c|c|c|c|c|}
\hline $\begin{array}{c}\text { ASIFT } \\
\text { Versions }\end{array}$ & Image size & $\begin{array}{c}\text { Feature Extraction } \\
\text { time (s) }\end{array}$ & $\begin{array}{c}\text { Matching } \\
\text { time (s) }\end{array}$ & $\begin{array}{c}\text { Total time } \\
\text { (s) }\end{array}$ & Speed-up \\
\hline \hline ASIFT-RR & $640 \times 480$ & 2.02 & 5.97 & 94.68 & 1.00 \\
\hline ASIFT-MEX & $640 \times 480$ & 1.54 & 3.55 & 60.55 & 1.56 \\
\hline ASIFT-GPU & $640 \times 480$ & 5.41 & 2.25 & 130.38 & 0.73 \\
\hline
\end{tabular}

TABLE 5.5: Time required and speed-up for modified ASIFT GPU when comparing to the other versions

and is almost 100x faster than the CPU based counterpart, so the SIFT operation being done within the ASIFT module should perform with a similar speed.

But after measuring the total run time of ASIFT, and comparing it with the MEX interfaced ASIFT described above, we have seen that the new ASIFT implementation containing CUDASIFT is actually slower in completing the same task than the MEX interfaced CPU based ASIFT, and surprisingly it's even slower than the implementation that used file read/write to communicate between the MATLAB and the $\mathrm{C}++$ modules.

This reduction in performance is due to the bottleneck being created by transferring the different image configurations of images between the host, which is essentially the CPU end, and device, which is the GPU end, of the GPU implementation.

As described in Chapter 4, for the GPU implementation we replaced the CPU based SIFT module with the GPU based CUDASIFT implementation. This GPU based CUDASIFT implementation transfers the images from host to device end, as any 
operation that is computed in the GPU and uses the SIMD parallel processing pipeline needs to be done using kernel functions that runs in the device end. But the image configurations that is part of how ASIFT achieves the "Affine-Invariant" title, is being done in the host end. This causes the ASIFT algorithm to transfer the warped image based on the tilt and rotation for every configuration that is being generated by ASIFT, and transferring this image back and forth is causing the algorithm to slow down. 


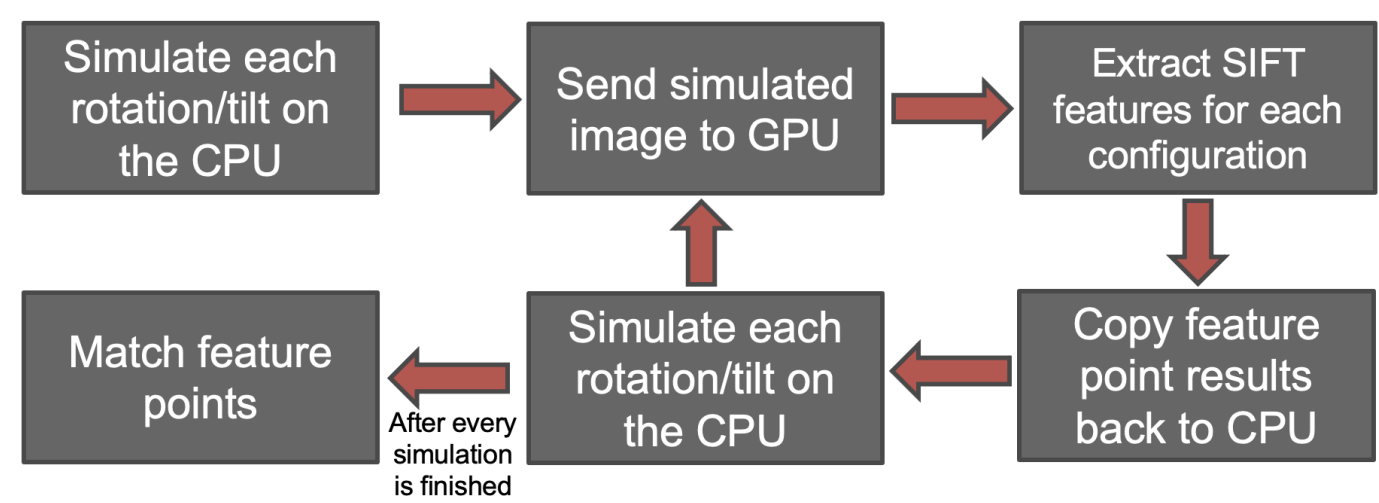

FIGURE 5.9: Block diagram of the ASIFT GPU based implementation, showing the bottleneck data transfers between the CUDA host and device for every SIFT operation, after simulating the images using the tilt and rotation configurations 


\subsection{Proposed Illumination-Matched Blending}

\subsubsection{Dataset Used}

I've used the Frog Mesentery sequence-3, with 491 individual frames, and sequence-4, with 770 individual frames, as well as the Malaria video sequences with 169 individual frames, to test the new methods investigated for this particular project. These three video sequences are chosen particularly for the difficulty they present for the current DNCC algorithm with the gradient/edge based gradient condition. For sequence- 3 and sequence4, the illumination difference can be a clear shift between illumination can be visualized from the resultant mosaic. The Malaria video sequence is the most challenging one of the set, which has structures that are not as visible as they are on the Mesentery dataset, and it has individual image frames that vary in illumination across that particular frame, which is very challenging for the illumination equalization techniques.

\subsubsection{Results for Illumination-Matching}

I've compared the three methods, Center-Weighing Feather Blending, Laplacian Pyramid Blending, and Intensity Matching, with the result from the DNCC paper using GRR condition and Replacement Blending on three video sequences. 
The first video-sequence I've tested is the Frog Mesentery Sequence-3, which contains 491 image frames, and intensity shifts are clearly visible in the mosaic created with the DNCC algorithm. 


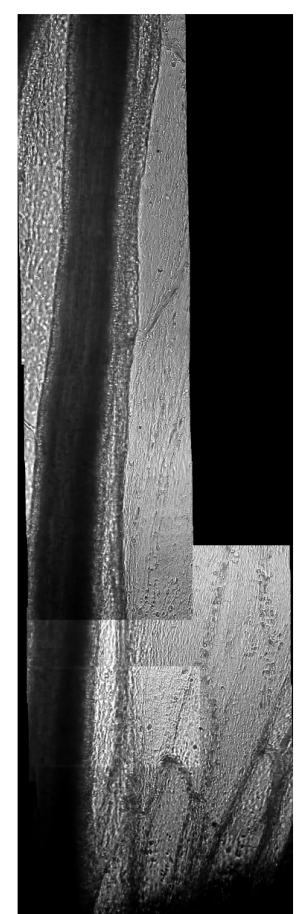

A - DNCC

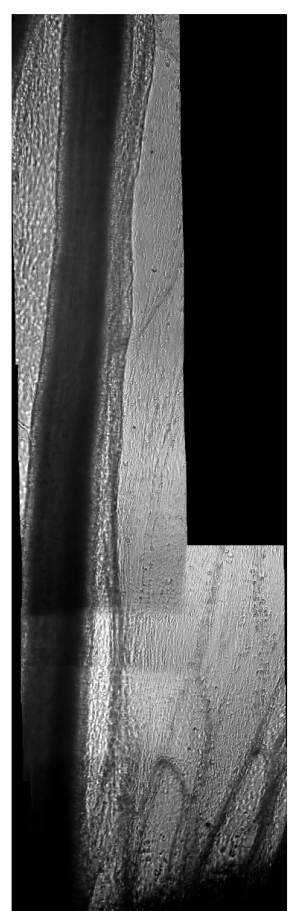

B - Center-Weighing (Feather Blending)

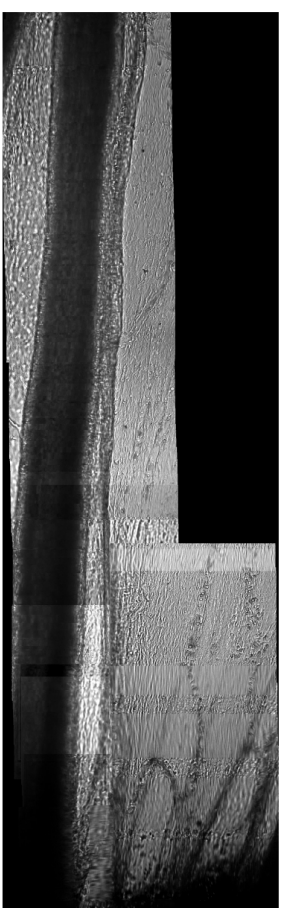

C- Laplacian Blending

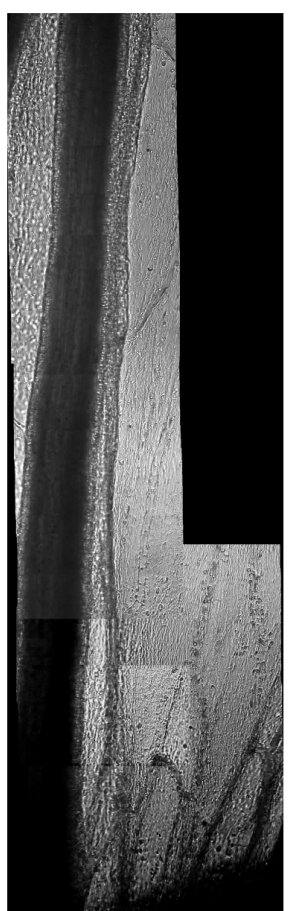

D - Intensity Matching

FIGURE 5.10: Showing the resulting mosaic from Frog Mesentery Sequence3 dataset using the four different blending algorithms. The mosaic marked as $A$ is generated using Rumana Aktar's DNCC algorithm [5] 
As can be seen from the image above, Center-Weighing method eliminates the rapid shift in the illumination by smoothing the intensity of the overlapped part, but still the illumination changes are visible from the mosaic and we can also see that the resultant mosaic is less sharp than the other three mosaic results.

The Laplacian Blending technique also smoothes out the shift of illumination, and keeps the sharpness of the mosaic, but it introduces some additional patches in the mosaic with illumination shifts, which is not desirable.

The Intensity Matching technique tries to match the intensity of the overlapped part for the subsequent image frames, and largely overcomes the illumination shift that of the original mosaic.

The results for Frog Mesentery Sequence-4 result shows similar mosaics with the four difference techniques. But it changes for the Malaria video sequence, as it contains image frames that have different illumination/intensity level in a single frame, rather than different illumination/intensity level for different frames (which Intensity Matching can overcome). This illumination shift in a single image frame causes visible banding effects for the Intensity Matching technique. Results for the two video sequence are added below: 


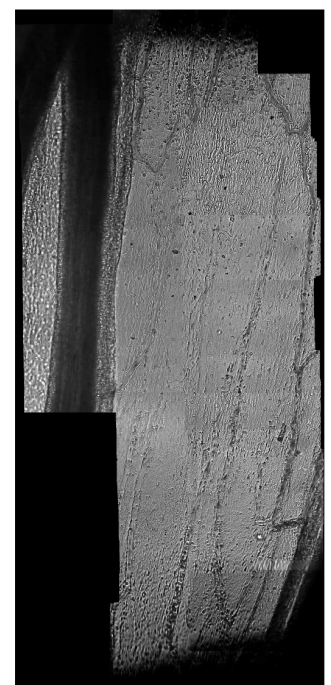

A- DNCC

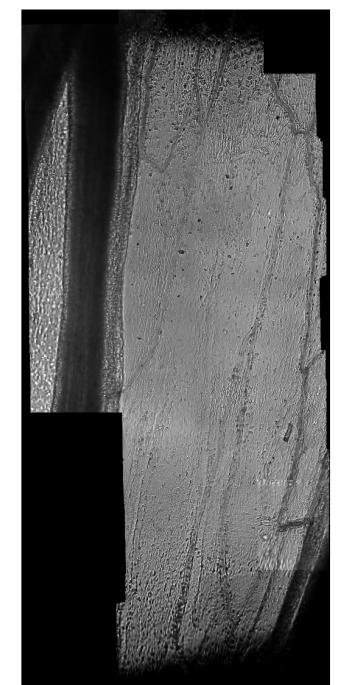

B - Center-Weighing (Feather Blending)

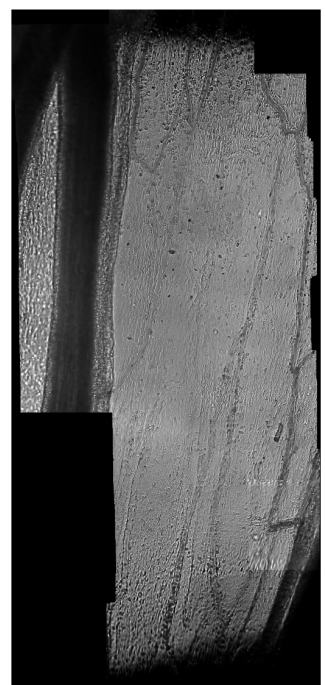

C- Laplacian Blending

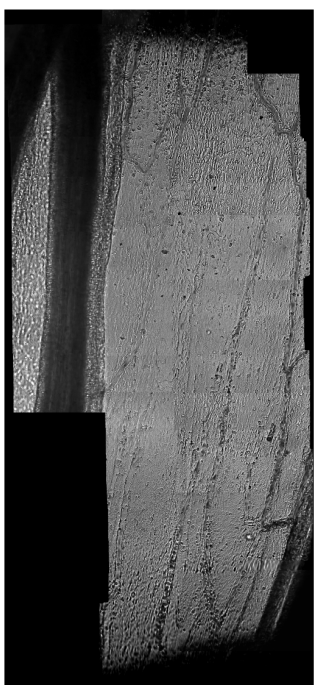

D - Intensity Matching

FIGURE 5.11: Showing the resulting mosaic from Frog Mesentery Sequence4 dataset using the four different blending algorithms. The mosaic marked as $A$ is generated using Rumana Aktar's DNCC algorithm [5] 
As mentioned above, we can see from figure-12 that the banding effects between subsequent frames are present in the mosaic generated using the Intensity-Matching technique, as the individual frames are brighter (higher intensity) at the center, but darker at the border region. This situation doesn't affect the Center-Weighing Feather Blending and Laplacian Blending techniques much, which is also evident from the results above. 

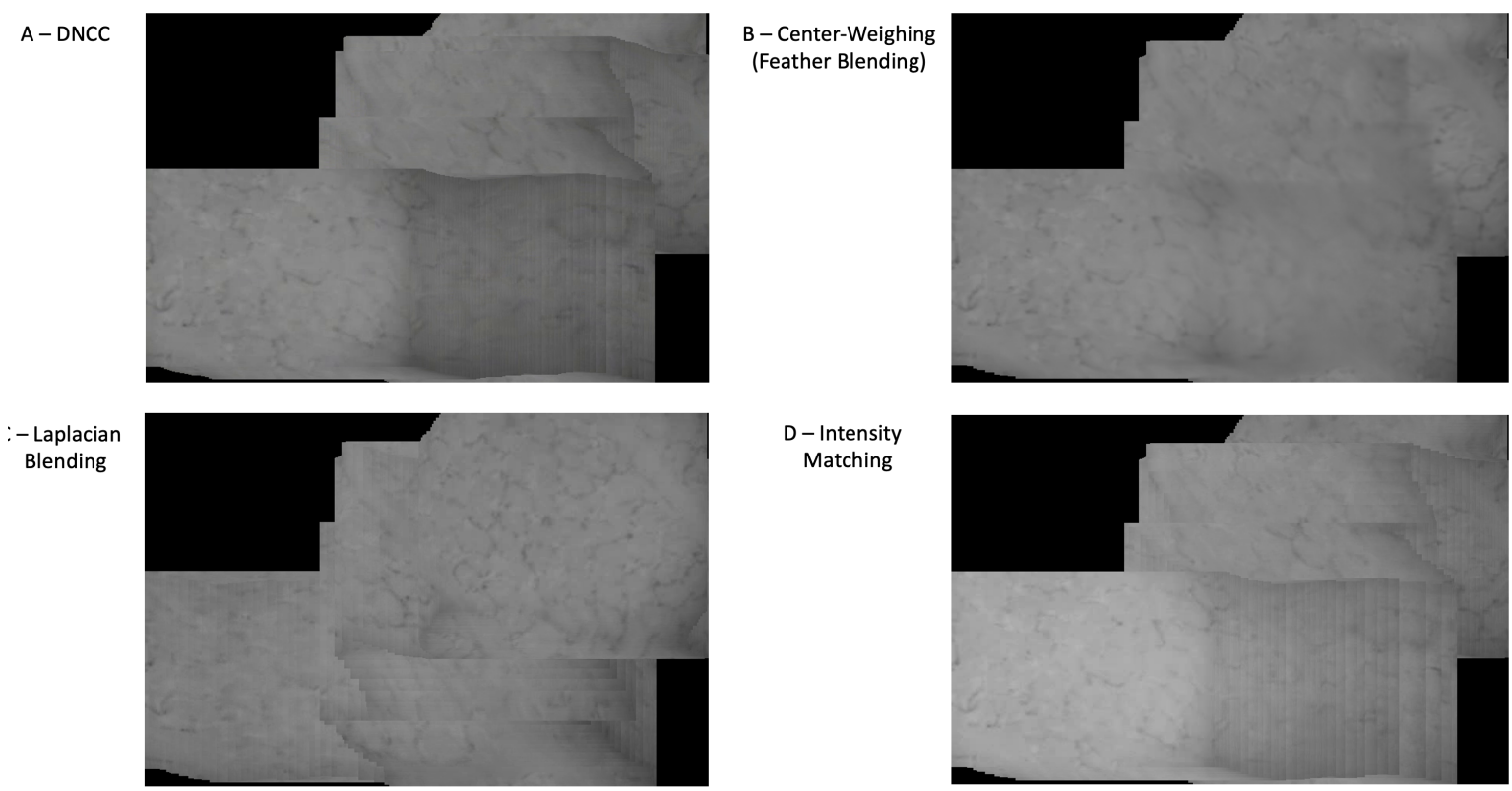

$$
\begin{gathered}
\text { D - Intensity } \\
\text { Matching }
\end{gathered}
$$

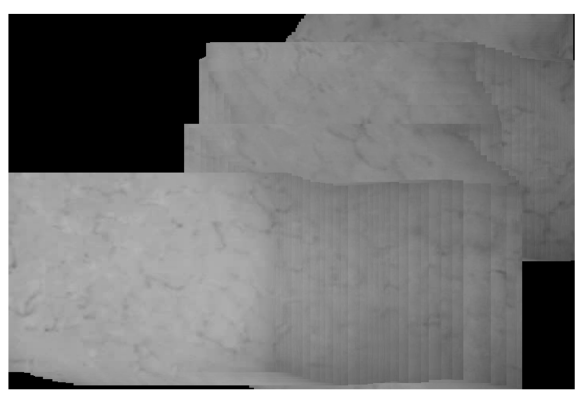

FIGURE 5.12: Showing the resulting mosaic from Malaria dataset using the four different blending algorithms. The mosaic marked as $A$ is generated using Rumana Aktar's DNCC algorithm [5] 


\subsubsection{Evaluation}

The visual results are also evident from the evaluation metrics results, shown in figure-13. As can be seen for the Frog Mesentery Sequence-3 and Sequence-4 results, the Intensity matching has the best scores for MSE (lower is better), PSNR (higher is better) and SSIM (higher is better). It also has the best score for MOID (lower is better) for Sequence-3. Though we can see some banding effects in the mosaic for Sequence- 4 with the intensity matching method, the MOID scores are thus higher for that particular method.

And as mentioned above, for the Malaria Dataset, due to the heavy banding effect caused by intensity difference in individual frames for the Intensity-Matching mosaic, Center-Weighing Feather Blending has the best scores for MSE and PSNR, and is also very close to the highest SSIM score. 


\begin{tabular}{|c|c|c|c|c|c|}
\hline Video Sequence & Method & MSE & PSNR & SSIM & MOID \\
\hline \hline \multirow{4}{*}{ Sequence3 } & DNCC & - & - & - & 28.27 \\
\cline { 2 - 6 } & Center-Weighing & 260.16 & 23.98 & 0.86 & 11.28 \\
\cline { 2 - 6 } & Laplacian & 1036.08 & 17.98 & 0.56 & 8.95 \\
\cline { 2 - 6 } & Intensity-Matching & $\mathbf{1 3 9 . 0 9}$ & $\mathbf{2 6 . 7 0}$ & $\mathbf{0 . 9 5}$ & $\mathbf{6 . 5 4}$ \\
\hline \multirow{4}{*}{ Sequence4 } & DNCC & - & - & - & 9.12 \\
\cline { 2 - 6 } & Center-Weighing & 392.57 & 22.19 & 0.81 & $\mathbf{2 . 3 4}$ \\
\cline { 2 - 6 } & Laplacian & 392.12 & 22.20 & 0.81 & 3.61 \\
\cline { 2 - 6 } & Intensity-Matching & $\mathbf{4 2 . 6 3}$ & $\mathbf{3 1 . 8 3}$ & $\mathbf{0 . 9 8}$ & 7.09 \\
\hline \multirow{4}{*}{ Malaria } & DNCC & - & - & - & 1.82 \\
\cline { 2 - 6 } & Center-Weighing & $\mathbf{9 7 . 1 0}$ & $\mathbf{2 8 . 2 6}$ & 0.95 & 0.72 \\
\cline { 2 - 6 } & Laplacian & 219.13 & $\mathbf{2 4 . 7 2}$ & 0.89 & 0.85 \\
\cline { 2 - 6 } & Intensity-Matching & 517.89 & $\mathbf{2 0 . 9 9}$ & $\mathbf{0 . 9 8}$ & $\mathbf{0 . 5 0}$ \\
\hline
\end{tabular}

TABLE 5.6: Results showing evaluation metrics and corresponding scores the the mosaics created with the different methods discussed. Mosaic created with Gradient-Blending [5] method is counted as the ground-truth, thus MSE, PSNR and SSIM values are not present 
Multiple methods are investigated and an algorithm is proposed, which can be incorporated with the VMZ mosaic generation pipeline to create a mosaic that handles the illumination difference between subsequent frames better than the regular replacement blending technique. The blending methods shown for this project, especially the Center-Weighing blending overcomes the banding effect caused by sudden illumination changes in subsequent frames, as well as illumination difference seen in different parts of an individual frame, where the replacement blending and the illumination-matching algorithm fails to mitigate the particular banding effect.

Though Gradient Response Ratio is used for skipping the frames considered to be blurry, the blending methods still performs worse than Replacement-Blending in SSIM, as they try to blend the overlapped part of the new frame with the Mosaic, creating a blurry result due to Deformable structure.

Taking the Mean from all overlapped pixels might be causing an issue, as the structures are deformable and thus the same pixels might not align, feature matched points in the overlapped part might be considered as a better alternative. This is an avenue which might be expanded on in the future.

I've also shown that the Intensity-Matching performs better than using the regular replacement blending, and can mitigate 
the illumination difference in subsequent frames nicely. The Center-Weighing and Laplacian-Pyramid blending might also be a viable solution when there are visible illumination differences in different parts of the same image frame.

\subsection{Overall Performance Improvement of VMZ}

\subsubsection{Updated Code-Profiling}

After integrating the improved MEX-interfaced modules with the existing VMZ MATLAB pipeline, we have timed the whole VMZ pipeline. We have performed 4 different set of test runs for 4 different versions of the VMZ pipeline, the first one being the initial VMZ MATLAB workflow, and the last the most improved VMZ pipeline that contains the MEX-interfaced Multi-Threaded implementation of NCC, and the MEX-interfaced modified ASIFT implementation which has the redundant operations removed. The second version in this test contains the MATLAB implemtation of NCC but the improved ASIFT module, and the third VMZ version has the CPU based Multi-Threaded NCC with the ASIFT version that has the redundancy operations removed. 


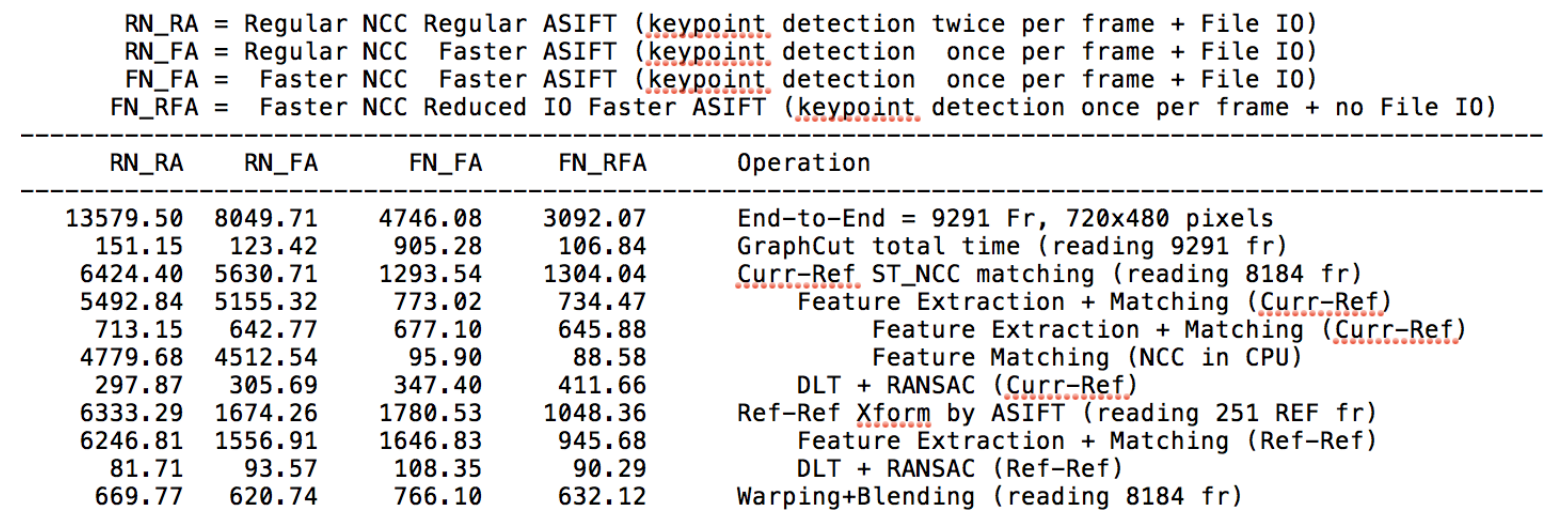

FIGURE 5.13: Improved Code Profiling showing the required time for each individual module for the VMZ pipeline after integrating improved modules, courtesy of Rumana Aktar. This profiling contains timing for four (4) different VMZ pipeline, as specified in the profiling. The pipeline marked $F N \_R F A$ includes both the multithreaded NCC MEX implementation and the improved ASIFT MEX implementation 
As can be seen from the updated code-profiling after integrating the improved MEX-interfaced modules with VMZ, the time required for the last version, that includes the CPU Based Multi-Threaded NCC and Modified ASIFT with removed redundant operations, takes 3092.07 seconds, or 51.53 minutes, or 0.86 hours, instead of 3.78 hours that VMZ took initially to run over the VIRAT dataset tape2_8. 


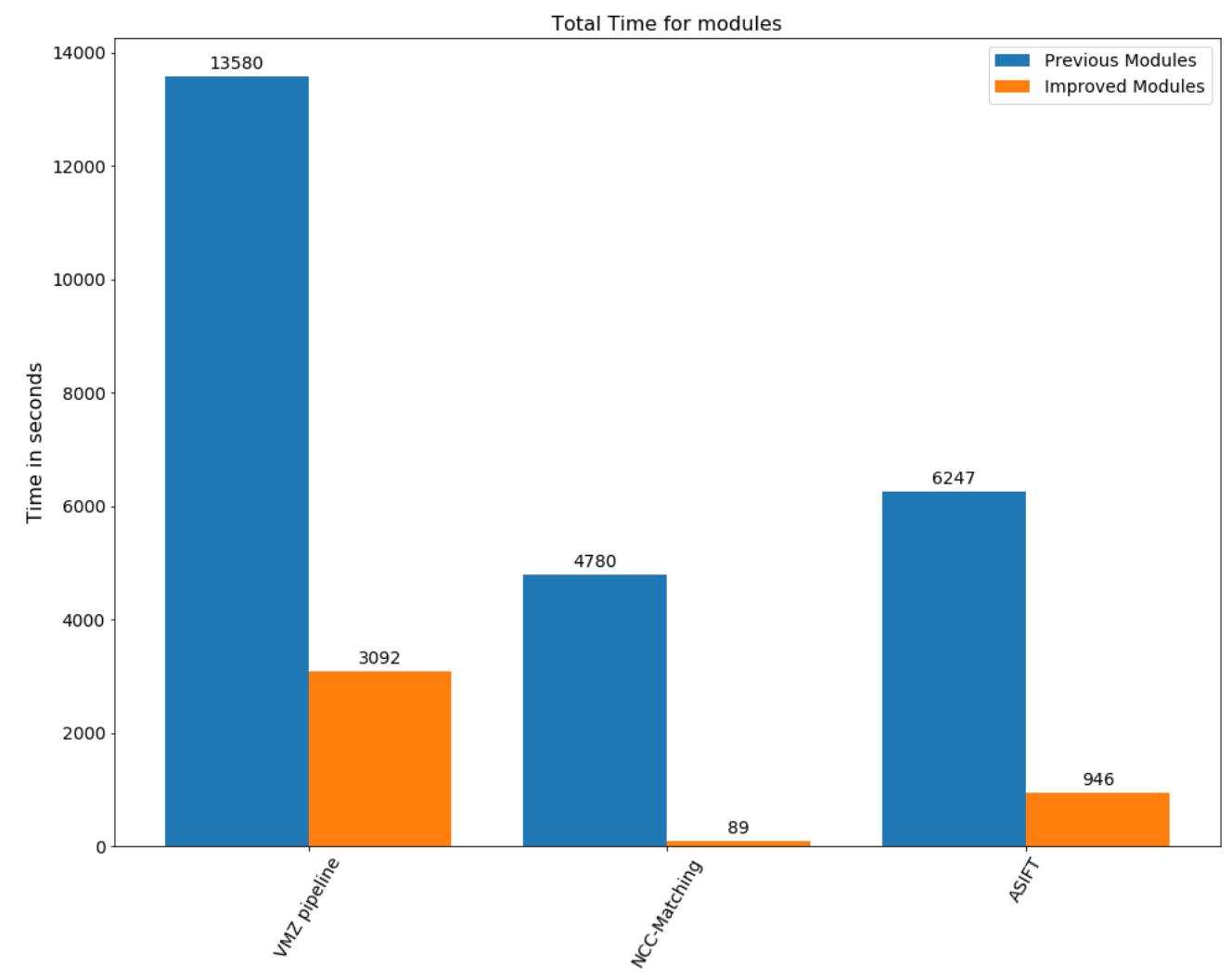

FIGURE 5.14: Improvement in total time required for overall VMZ pipeline and individual improved modules, showing both required time for the previous implementation and for the improved implementation for overall VMZ pipeline, NCC Matching, and Affine Invariant SIFT 


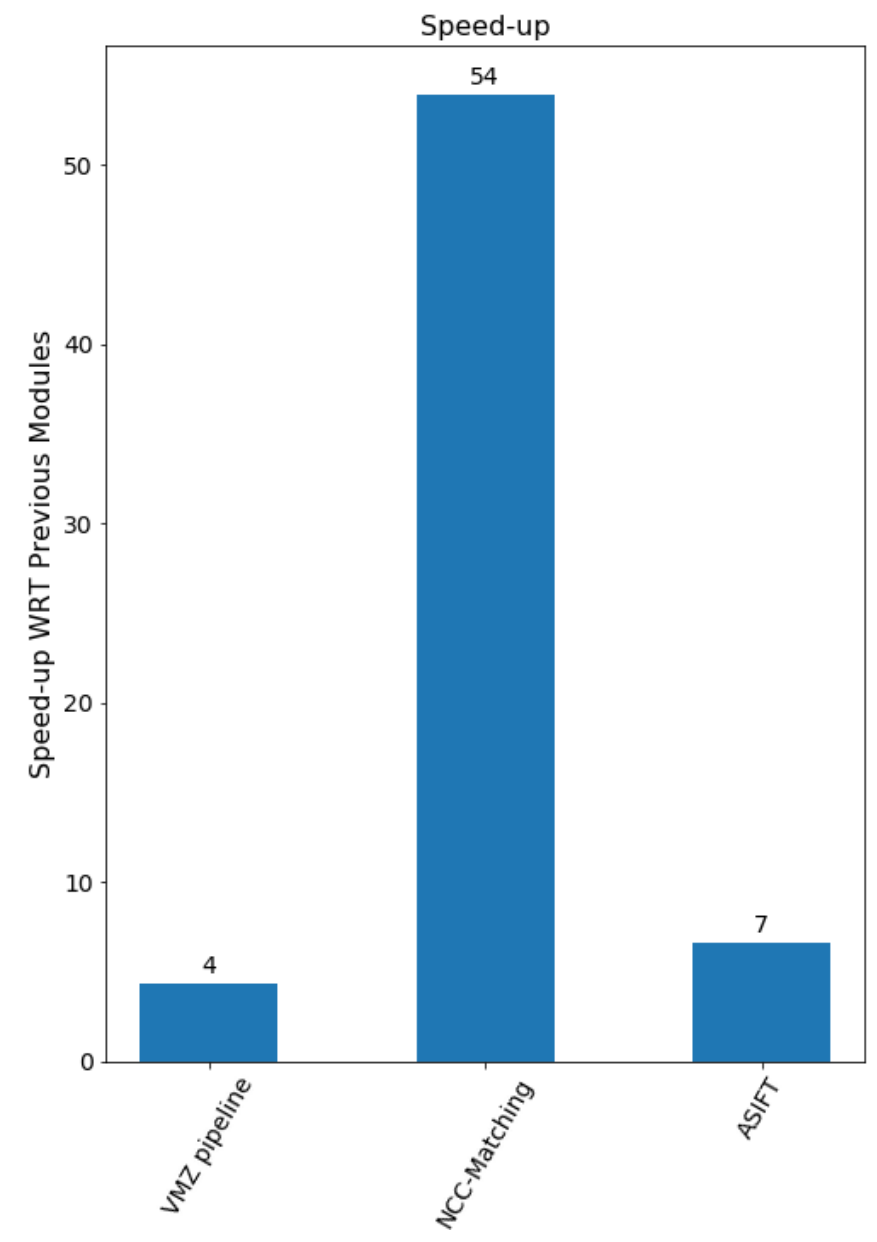

FIGURE 5.15: Speed-up for overall VMZ pipeline, and individual modules, as NCC Matching and Affine Invariant SIFT 


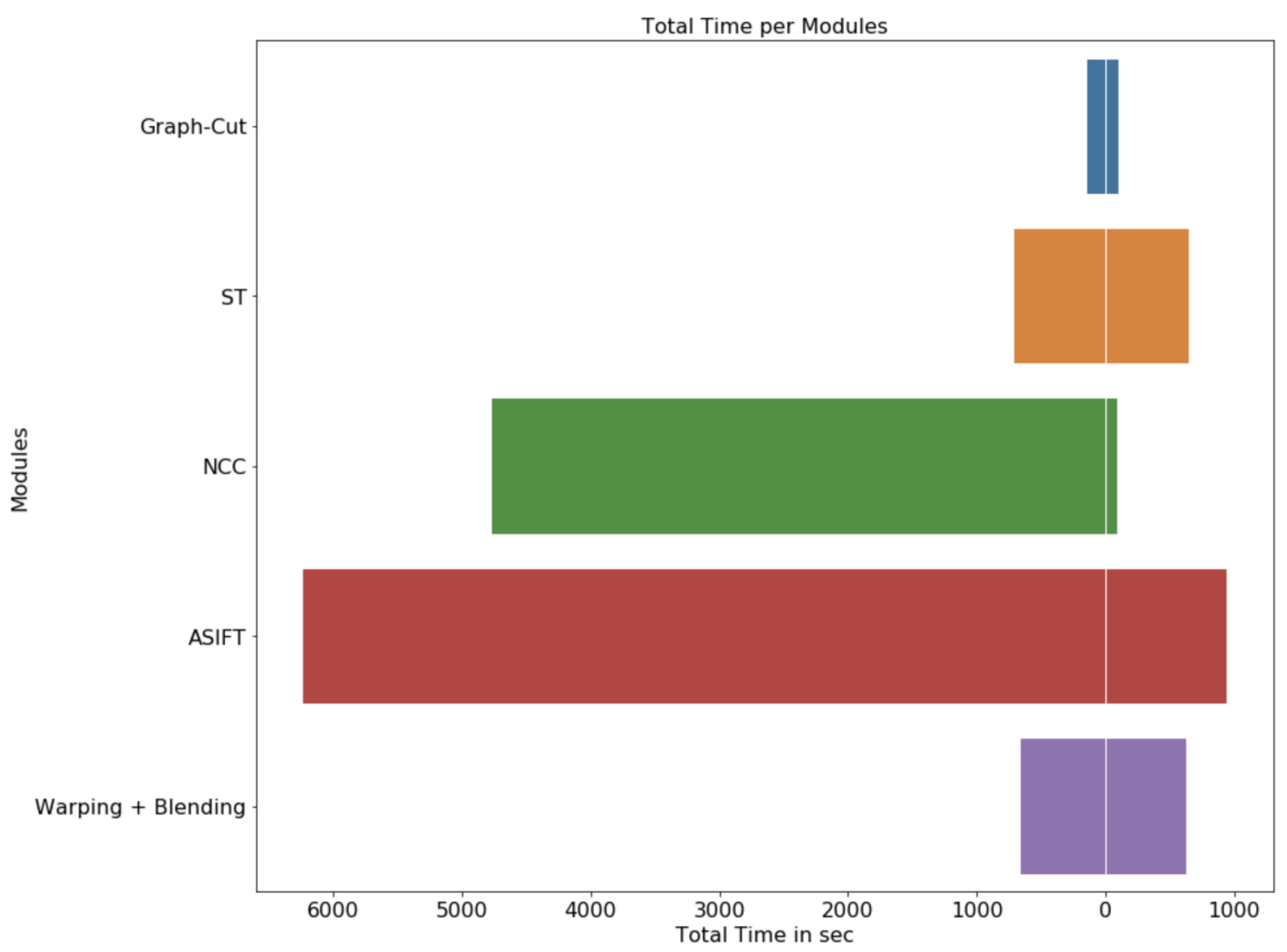

FIGURE 5.16: The pyramid plot shows the total time per modules, for both before and after integrating the improved modules to $\mathrm{VMZ}$, focusing on the total time differences for NCC module, which shows a 54x speed-up, and ASIFT module, which shows a $6.6 \mathrm{x}$ speed-up 


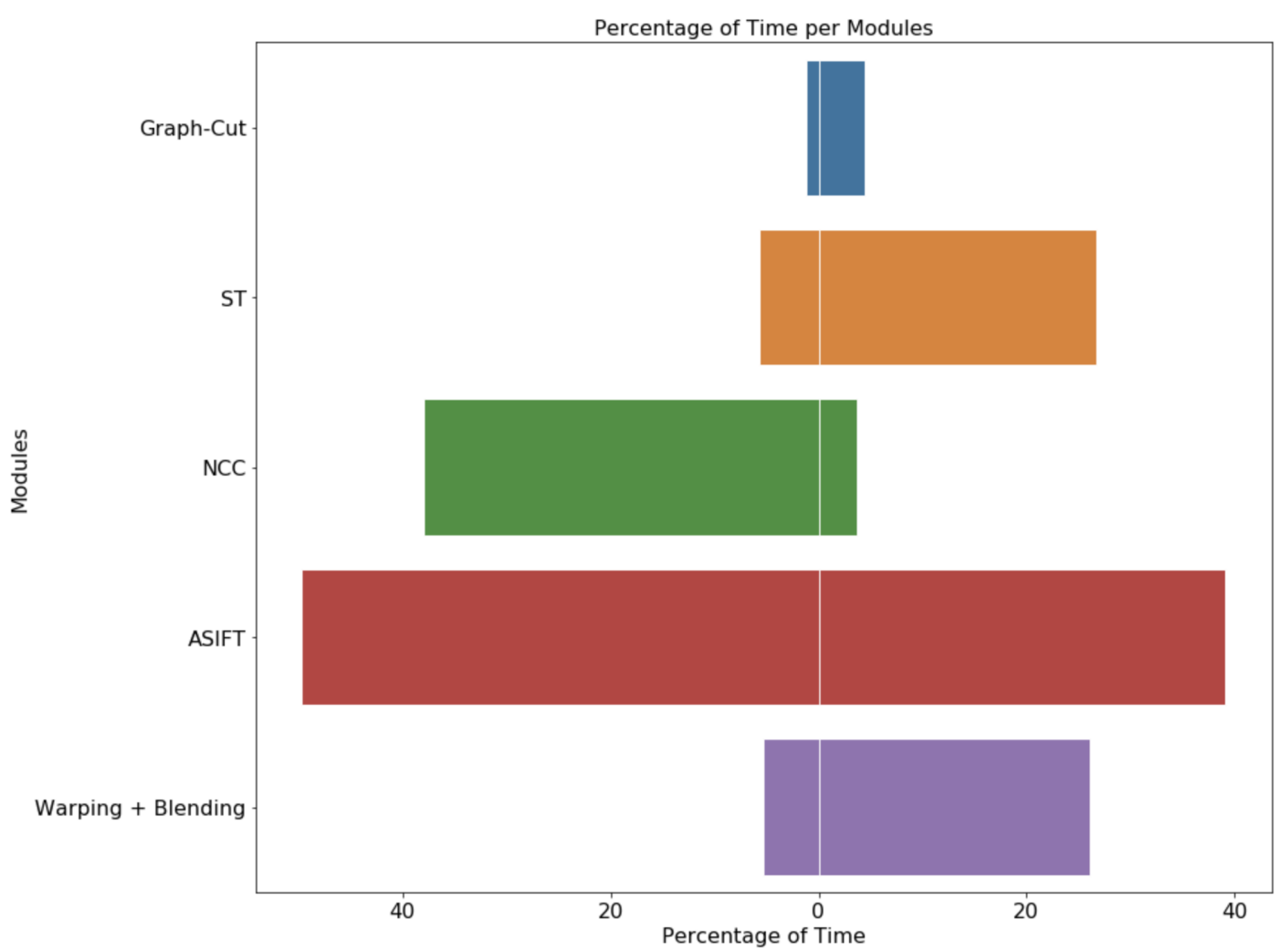

FIGURE 5.17: Pyramid plot for Percentage of time for each individual modules, left of zero shows percentage taken before and right side of zero shows after integrating the improved modules into VMZ/ From here we can see that previously the bottleneck were the NCC, and the ASIFT module, where the NCC module is taking the minimum amount of time among all of the modules. The ASIFT is still the most compute intensive module though, even after a speed-up of $6.6 \mathrm{x}$ 


\subsubsection{Updated Code Architecture for VMZ}

The updated code architecture for VMZ contains the improved $\mathrm{C}++$ modules, leveraging MEX-interfacing, Multi-Threading and modifications for speeding up the overall run-time of the VMZ pipeline. As can be seen from the code architecture, the improved Normalized Cross-Correlation module is integrated in place of the MATLAB call for NCC, using normxcorr 2, and is being called from the getHomographyMatrix function in the VMZ pipeline. As for the improved ASIFT module, it is still being called by both

Current_to_Reference and Reference_to_Reference, but the actual call to the ASIFT function has been broken down into two stages. For the first stage, only the detection operation is called for the images that are being processed to store the extracted feature-point list for each image. And on the next stage, the matching operation is called over a pair of extracted featurepoint list. 


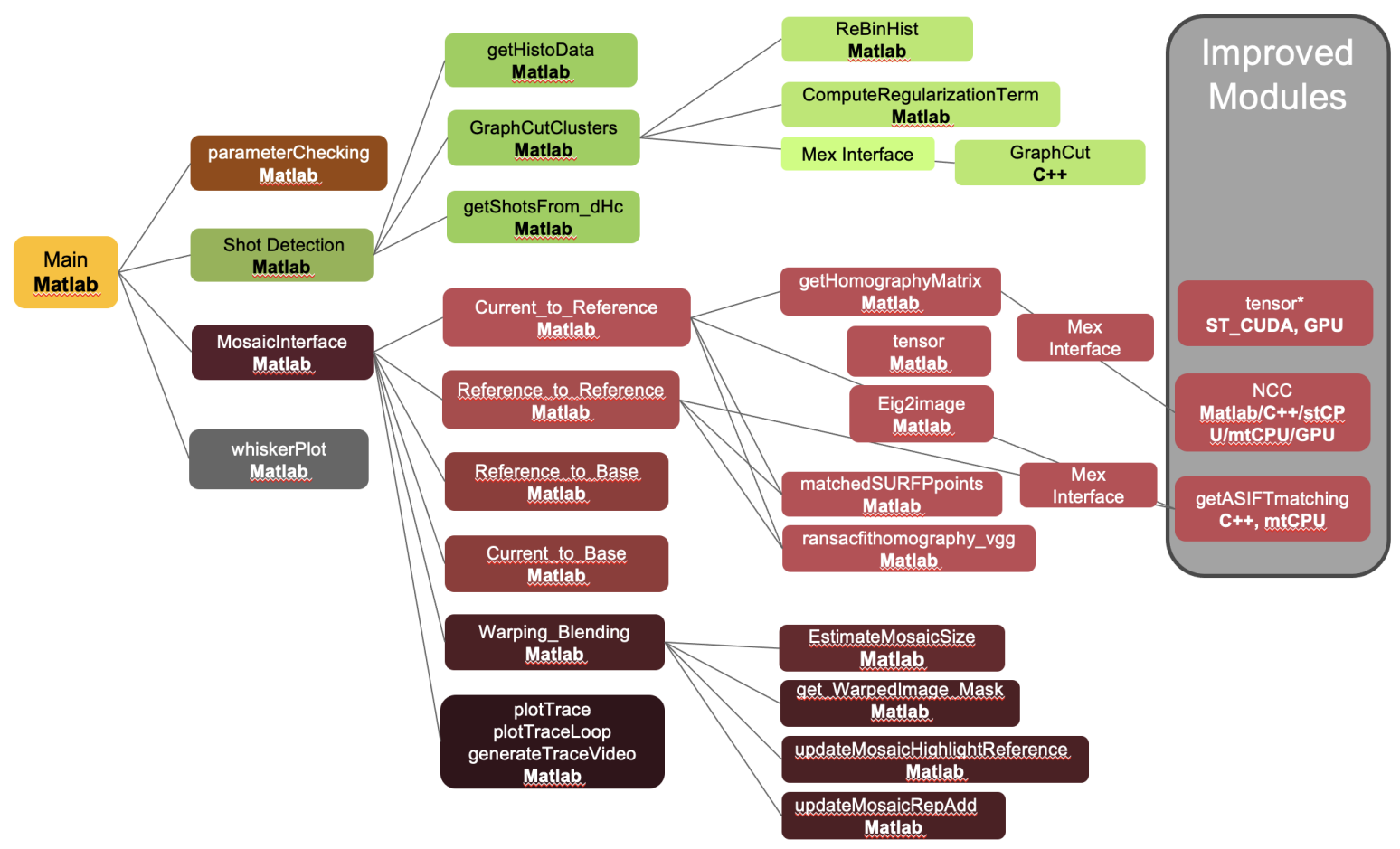

FIGURE 5.18: Updated Code Architecture for VMZ pipeline showing the improved modules that are implemented in $\mathrm{C}++$ and integrated with the MATLAB pipeline using the MEX interfacing 


\section{Chapter 6}

\section{Additional Contributions and Future Directions}

\subsection{Additional Contribution}

\subsubsection{Meta-Mosaic Generation}

Our Video Mosaicing and Summarization pipeline generates multiple mini-mosaics for a single video sequence. The most frequent dataset we have used throughout this thesis is the VIRAT dataset tape2_8, which contains 9232 image frames. The shot boundary module in VMZ splits the image frames of this dataset into 10 shots, which essentially generates 10 mini-mosaics, each containing the image frames up until the next shot boundary. The next focus target of this pipeline is to join multiple generated mini-mosaics to create a more broad mosaic, which can also be called a meta-mosaic.

To create such a meta-mosaic, I have divised an algorithm 
that uses the the mini-mosaics the the matching information between each of the mini-mosaics, which contains number of inliers and percentage of inliers among the total number of matches. My algorithm creates a graph using these information, where the mini-mosaics are considered as the nodes of the graph with edges connecting one particular node to every other node in the graph. The number of inliers are considered as the cost of an edge between two nodes (or mini-mosaics). After creating such a graph, I used the MaximumSpanningTree algorithm to compute a Tree where all of the mini-mosaics are connected with each other, either directly or through a number of other nodes, and it guarantees that the total number of inliers will always be maximized in this case.

Initially we tried to run the algorithm as described above, where the mini-mosaics are considered as the nodes. But as we are using Krushkal's Algorithm [add-reference-here] which has a time complexity of $O(\lg N+\lg M)$ where $N$ is the number of nodes and $M$ is the number of edges, we have a lot of headroom to expand the number of nodes and edges before this algorithm requires a significant amount of run-time.

We expanded our test by also using the reference frame from each shot as the nodes, and is connected to all other reference frames through an edge. The number of inliers among all of the 
other reference frames are computed, and they act as the cost for their corresponding edges.

\subsubsection{Sequential tiling and Multi-Threaded image reading for VB3D}

Video-based 3D reconstruction, or VB3D [11] in short, is a tool developed by our lab members that can reconstruct city-scale 3D models upto a few square kilometers by using multiple camera views and a volumetric voting-based surface likelihood estimation technique. Current implementation of VB3D is based on CUDA GPU, and the amount of VRAM available for the GPU in use limits the algorithm to generate upto a certain resolution for the 3D model.

To overcome this issue, and to generate a higher resolution 3D model while still using the same amount of VRAM, I have modified the VB3D workflow and implemented a sequential 3D model generation by dividing up the model in multiple tiles. This tiling technique can be broken up arbitrarily and the amount of tiles can be set in the implementation. After generating the 3D model for each tile, all of the memory used for generating the model for the current tile are freed up before the process moves on to the next tile, thus at a time, this approach only uses the memory required to generate only a single tile. For our testing, 
we have generated a model with double the resolution that was being generated previously, by using 4 tiles.

I have also divised a multi-threaded approach for the image reading and preprocessing pipeline of VB3D. This multithreaded approach uses a publisher - subscriber model, where we create two types of threads. One type is thought of as the publisher, and for our use-case the publisher threads reads images off a specified directory and submits these images into a queue. The other type of threads are thought of as the subscriber, that keeps polling the said queue for any available images and as one of them finds an image it pops the image out of the queue and continues with the preprocessing. This is also described in the algorithm 6 below.

\subsubsection{Investigating PopSift and CudaSift for Benchmarking}

I have also investigated two GPU based detection and matching algorithms that are a variant of the popular SIFT algorithm, PopSift [12] and CudaSift [7]. These algorithms are used to create a benchmark where different variants of the SIFT algorithm is compared with each other on the basis of time required for individual detection and matching methods, the number of matches found, and the number of inliers and outliers within the found 


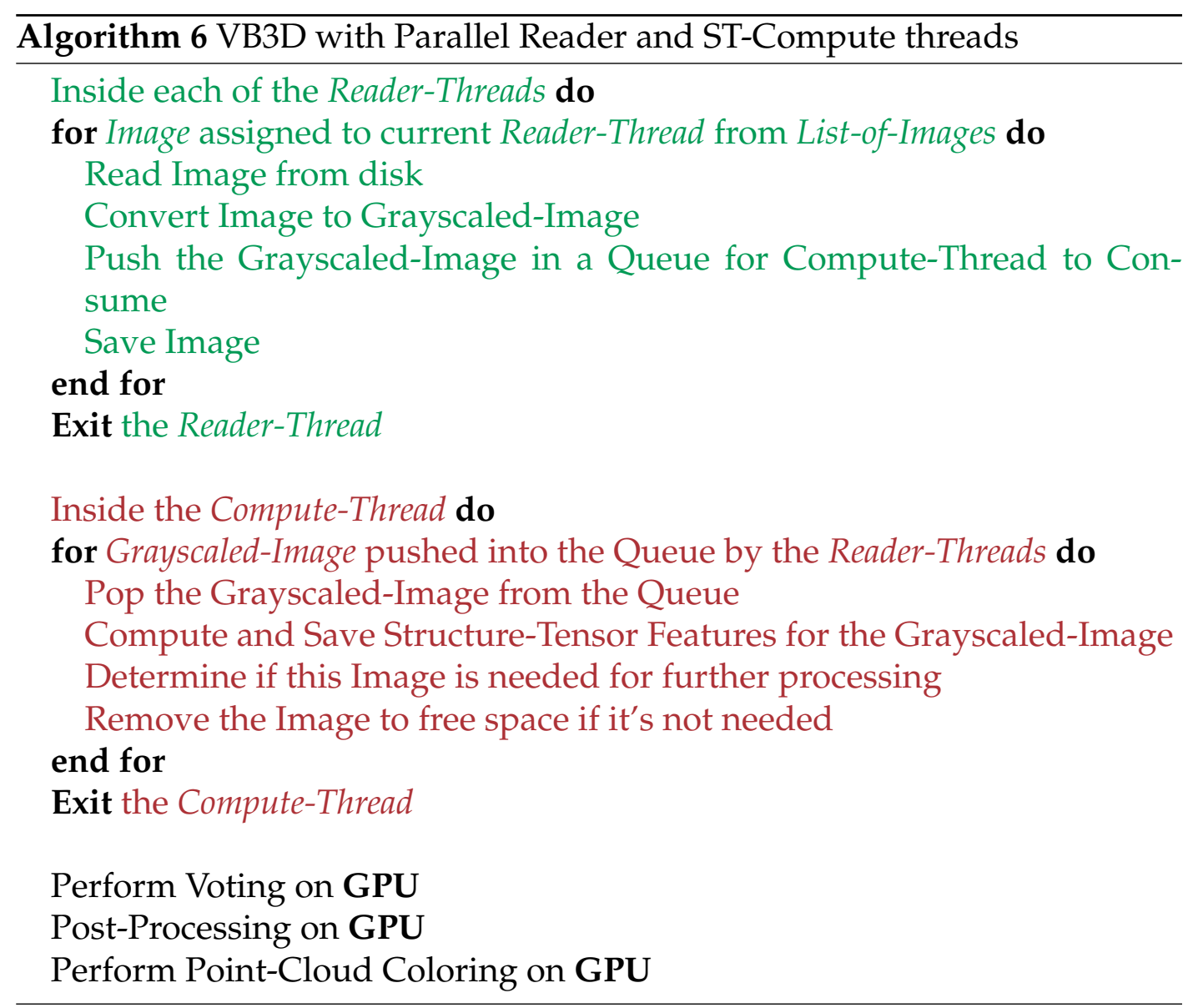

matches. We have used the the well known Oxford Affine Covariant Features Datasets for this benchmarking and generated our results using the images from that dataset.

PopSift was unusually tricky for generating the results, as it doesn't retain or output the matched feature points or coordinates, and instead outputs the index of the feature descriptors that are generated on the detection part of the algorithm. PopSift also uses a multi-threaded asynchronous pipeline for all of it's detection and matching tasks, and as all of the operations is done on the GPU device, the results are also on the device end 
and not on the host end of the workflow (for CUDA programming, device represents the actual GPU, where host represents the CPU part and any work done or data stored on the device end must be copied over to the host end before any further processing can be done in the host). After a task is submitted to the asynchronous thread, that can be either a detection task or a matching task, I added a checker to poll the threads and check if the task has been completed, and pull down the results from device to host after the task is finished. This result is then modified as needed for our benchmarking tool and outputted to an external file for further processing.

\subsection{Future Directions}

\subsubsection{Meta-Mosaic Generation}

Meta-Mosaic or Global Mosaic generation is the next step for our Video Mosaicing and Summarization pipeline, as this will enable the system to generate a global map of the object of interest, instead of generating multiple pieces as being done now with the mini-mosaics. This can be a challanging task as the Maximum Spanning Tree algorithm can generate a path to stitch together the mini-mosaics to form a meta-mosaic but the calculated homography for stitching two mini-mosaics gives a poor 
result, as a consequence the resulting stitched mini-mosaics are not placed correctly in the same plane as expected.

\subsubsection{ASIFT - GPU based implementation}

Affine-Invariant SIFT, or ASIFT is a very important feature detection and matching algorithm as it can detect features in a given homogeneous sequence even when all other feature descriptors fail to produce a good matching result. The current GPU implementation of ASIFT is running into a bottleneck where each configuration is begin generated on the host end, which is essentially being run on the CPU, of the code and then sent to the device end, which is run on GPU, for computing SIFT detection and matching. This whole pipeline needs to be transferred over to the device end of the code-base to avoid this data transfer and GPU initialization bottleneck.

This can be achieved by following the block diagram shown in figure 6.1. First the original image is sent to the device (GPU) end from host, and this is the only time the image is being transferred from host to device. After that the different configurations of rotations and tilts are computed and corresponding images are generated and stored on the GPU end. This follows by a GPU implementation of SIFT, and as the simulated images are already available on the device end, there is no need for any 


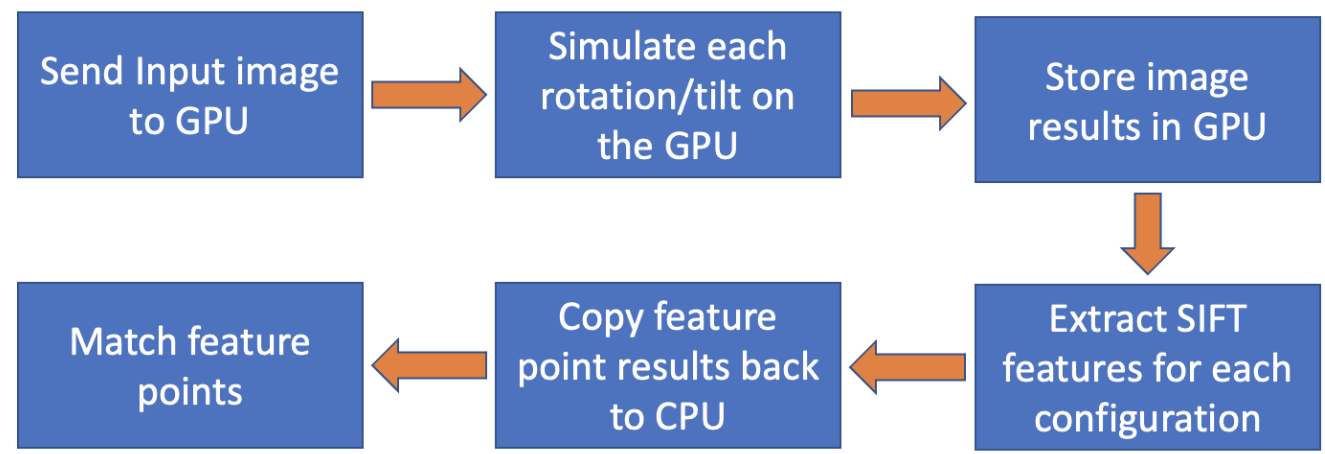

FIGURE 6.1: Figure shows the block diagram for future GPU implementation of ASIFT, where image transfer between device and host for every rotation/tilt configuration is reduced by generating the simulations on the GPU end

transfer between device and host. Finally the extracted feature points are copied back to the CPU and the matching operation is performed.

\subsubsection{Improved Structure Tensor - Integration with MATLAB}

The Structure Tensor improved module the leverages the GPU based parallelization using CUDA, which was discussed in this thesis, was not integrated with the VMZ pipeline, due to the MEX compiler not being able to compile the CUDA source files. As a future work, this improved module can be integrated with MATLAB for the VMZ pipeline.

\subsubsection{Porting of VMZ MATLAB modules in C++}

Porting of existing MATLAB modules in VMZ into a lower level language, like $\mathrm{C}++$, has been in focus, and the improvements in 
the modules described in this thesis has been a part of that goal. The driver code of the VMZ pipeline, especially the part that drives the feature detection, matching and homography estimation, still needs to modularized and converted. This is a very challenging task and thus needs to done in small steps, and with modularized testing framework, so that every part being converted can be tested against the original workflow to determine any difference in underlying libraries or bugs introduced while conversion.

The current plan towards reaching this goal is to first implement the main driver part of VMZ into Python, that is more manageable and will require a lot less development time than porting into $\mathrm{C}++$ all together.

\subsubsection{Camera Pose Estimation}

Currently Planar Homography is being used by the VMZ pipeline to determine the plane of each the mosaics before any warping and blending is applied to the mini-mosaic. Camera pose estimation can be a very effective alternative for detecting this homography analytically, with scenes that has a significant structure in it. 


\subsubsection{Support for Deep and Engineered Features}

The VMZ pipeline currently supports Normalized Cross-Correlation, Structure Tensor, SIFT, Affine-Invariant SIFT, and SURF features. But in the future it would be better for the pipeline to be able to support engineered feature descriptors, as well as feature descriptors using deep learning methods, which might perform better for a particular type of object of interest. 


\section{Chapter 7}

\section{Conclusions}

In this thesis I have demonstrated some ways to significantly speed up the computation of the compute intensive and long running modules of the Video Mosaicing and Summarization pipeline, namely Normalized Cross Correlation, Structure Tensor and Affine Invariant SIFT. Speed up has been gained for the modules using CPU based multi-threading, GPU based parallel processing, improved interfacing between different MATLAB and $\mathrm{C}++$ modules and modifying existing workflows towards more efficient usage of computational resources. Important modules that are part of many pipelines, including that of our own image mosaicing pipeline, VMZ, can no longer be such a significant bottleneck to the whole workflow. Furthermore, the difference between the NCC calculation of various sources (MATLAB and OpenCV) and Structure Tensor is not significant to warrant using the slower MATLAB modules. It is, however, 
still going to be one of the slower aspects of the system, as the actual computation is being done by these heavy modules. There is also room for improvement in terms of speed up, especially with the ASIFT GPU based parallel implementation, which has been part of our discussion in the previous chapter. Originally for the MATLAB implementation, the frame-rate for the VMZ pipeline was 0.68 frames per second. After integrating the improved NCC and ASIFT modules discussed in this thesis, a significant improvement has been gained in this, and the current frame-rate for the VMZ pipeline gone up to 3.01 frames per second. In this direction, to achieve the goal of generating minimosaics in real-time, this frame rate will need to go upto 30 frames per second.

I have also proposed a novel illumination-matching blending algorithm that can be used for creating image mosaics from image frames that has different illumination intensities in it's different frames. Subsequently, I have also proposed a novel evaluation metric (MOID) that can determine the quality of a generated mosaic based on illumination differences in building frames. The proposed algorithm generates image mosaics with less illumination variance and a better score than compared methods for the MOID metric. But the algorithm struggles to 
match illumination when a single frame in the input image sequence contains illumination variance, and there is room for improvement for this particular case. 


\section{Bibliography}

[1] Nafis Ahmed et al. "GPU and multi-threaded CPU enabled normalized cross correlation". In: Geospatial Informatics X. Ed. by Peter J. Doucette et al. Vol. 11398. International Society for Optics and Photonics. SPIE, 2020. DOI: 10.1117/12.2561529, URL: https://doi .org/10.1117/12. 2561529 .

[2] R. Aktar et al. "Geospatial content summarization of UAV aerial imagery using mosaicking". In: SPIE Geospatial Informatics, Motion Imagery, and Network Analytics VIII. Vol. 10645. International Society for Optics and Photonics. 2018, p. 106450I.

[3] R. Aktar et al. "Performance Evaluation of Feature Descriptors for Aerial Imagery Mosaicking". In: IEEE Applied Imagery Pattern Recognition Workshop (AIPR). 2018, pp. 1-7.

[4] R. Aktar et al. "Video haze removal and poisson blending based minimosaics for wide area motion imagery". In: IEEE Applied Imagery Pattern Recognition Workshop (AIPR). 2016, pp. 1-7.

[5] Rumana Aktar et al. "Mosaicing of Dynamic Mesentery Video with Gradient Blending". In: 2020 IEEE International Conference on Image Processing (ICIP). 2020, pp. 563-567. DOI: 10 . 1109 / ICIP40778 . 2020 . 9191045 
[6] Sarala Arunagiri and Jaime Jaloma. "Parallel GPGPU stereo matching with an energy-efficient cost function based on normalized cross correlation". In: Image Processing: Algorithms and Systems XI. Vol. 8655. International Society for Optics and Photonics. 2013, p. 86550X.

[7] Mårten Björkman, Niklas Bergström, and Danica Kragic. "Detecting, segmenting and tracking unknown objects using multi-label MRF inference". In: Computer Vision and Image Understanding 118 (2014), pp. 111127. ISSN: 1077-3142. DOI: https://doi . org/10 .1016/ j . cviu . 2013. 10.007. URL: https://www . sciencedirect . com/science/article/ pii/S107731421300194X.

[8] Kai Briechle and Uwe D Hanebeck. "Template matching using fast normalized cross correlation". In: Optical Pattern Recognition XII. Vol. 4387. International Society for Optics and Photonics. 2001, pp. 95-102.

[9] Y. Fouda and K. Ragab. "An efficient implementation of normalized cross-correlation image matching based on pyramid". In: IEEE International Joint Conference on Awareness Science and Technology Ubi-Media Computing (iCAST 2013 UMEDIA 2013). 2013, pp. 98-103.

[10] Durgaprasad Gangodkar et al. “Efficient Variable Size Template Matching Using Fast Normalized Cross Correlation on Multicore Processors". In: Advanced Computing, Networking and Security. Ed. by P. Santhi Thilagam et al. Berlin, Heidelberg: Springer Berlin Heidelberg, 2012, pp. 218227. ISBN: 978-3-642-29280-4.

[11] Ke Gao et al. "Sensitivity of Multiview 3D Point Cloud Reconstruction to Compression Quality and Image Feature Detectability". In: Oct. 2019, pp. 1-6. DOI: 10.1109/AIPR47015.2019.9174580.

[12] Carsten Griwodz, Lilian Calvet, and Pål Halvorsen. "Popsift: A Faithful SIFT Implementation for Real-Time Applications". In: Proceedings of the 9th ACM Multimedia Systems Conference. MMSys '18. Amsterdam, 
Netherlands: Association for Computing Machinery, 2018, pp. 415-420. ISBN: 9781450351928. DOI: 10 . 1145/3204949 . 3208136, URL: https : //doi.org/10.1145/3204949.3208136

[13] Adel Hafiane, Kannappan Palaniappan, and Guna Seetharaman. "UAVvideo registration using block-based features". In: IEEE International Geoscience and Remote Sensing Symposium (IGARSS). Vol. 2. 2008, pp. II1104.

[14] JP Lewis. “Fast Normalized Cross-Correlation. 2001". In: Industrial Light and Magic ().

[15] Tony Lindeberg. "Scale invariant feature transform". In: (2012).

[16] Jakob Santner et al. "PROST: Parallel robust online simple tracking". In: IEEE Conf. on Computer Vision and Pattern Recognition. 2010, pp. 723730.

[17] Richard Szeliski. Image Alignment and Stitching: A Tutorial. Tech. rep. Microsoft Research, Dec. 2010.

[18] "Template Matching, Image Processing Module". In: OpenCV Documentation https://docs . opencv . org/2.4/doc/tutorials/imgproc/ histograms/template_matching/template_matching.html ().

[19] Carnegie Mellon University. "Image Blending Slides". In: http : // graphics . cs . cmu . edu / courses / 15-463/2008_ fall / Lectures / blending.pdf. 2008.

[20] Xiaotao Wang and Xingbo Wang. "FPGA-based parallel architectures for normalized cross-correlation". In: IEEE Int. Conf. on Information Science and Engineering. 2009, pp. 225-229.

[21] Jae-Chern Yoo and Tae Hee Han. "Fast normalized cross-correlation". In: Circuits, Systems and Signal Processing 28.6 (2009), p. 819. 
[22] Guoshen Yu and Jean-Michel Morel. "ASIFT: An Algorithm for Fully Affine Invariant Comparison". In: Image Processing On Line 1 (2011). https://doi.org/10.5201/ipol.2011.my-asift, pp. 11-38.

[23] Zhe Zhu et al. "A Comparative Study of Algorithms for Realtime Panoramic Video Blending". In: IEEE Transactions on Image Processing 27.6 (2018), pp. 2952-2965. DOI: 10.1109/TIP. 2018.2808766. 